Tohoku Math. J.

55 (2003), 207-254

\title{
VARIATIONAL PROBLEMS OF NORMAL CURVATURE TENSOR AND CONCIRCULAR SCALAR FIELDS
}

\author{
KUNIO SAKAMOTO
}

(Received May 14, 2001, revised August 5, 2002)

\begin{abstract}
We consider the integral of (the square of) the length of the normal curvature tensor for immersions of manifolds into real space forms, especially into spheres. The first variation formula is given and the Euler-Lagrange equation is expressed in terms of the isothermal coordinates when the submanifold is two-dimensional. The relations between the critical surfaces and Willmore surfaces are discussed. We also give formulas concerning the residue of logarithmic singularities of $S$-Willmore points or estimate it by a conformal invariant.

We show that if a compact critical surface satisfies certain conditions and the immersion is minimal, then the Gauss curvature is a non-negative constant and the immersion is a standard minimal immersion of a sphere or a constant isotropic minimal immersion of a flat torus. To prove this result, we study two-dimensional Riemannian manifolds admitting concircular scalar fields whose characteristic functions are polynomials of degree 2. Moreover, the case that the characteristic functions are polynomials of degree 3 is studied.
\end{abstract}

Introduction. In the 1960's, Willmore proposed studying the functional

$$
\mathcal{L}[\phi]=\int_{M}\left(\eta^{2}-K\right) d v
$$

on the space of immersions $\phi: M \rightarrow \boldsymbol{R}^{3}$ of a compact orientable surface $M$ into a threedimensional Euclidean space $\boldsymbol{R}^{3}$, where $\eta$ is the mean curvature of $\phi, K$ the Gauss curvature of the induced metric and $d v$ the volume element. The functional $\mathcal{L}[\phi]$ is called the Willmore functional and a critical surface is called a Willmore surface.

Around 1980, Bryant [5] studied Willmore surfaces in a three-dimensional sphere $S^{3}$ and contributed to the subject. He defined a conformal Gauss map of a surface $M$ in $S^{3}$ into the de Sitter space of all oriented small spheres of $S^{3}$ and showed that $M$ is a Willmore surface if and only if the conformal Gauss map is harmonic. Furthermore, he obtained a duality theorem for Willmore surfaces in $S^{3}$. Ejiri [11] introduced the notion of $S$-Willmore surfaces and generalized Bryant's duality theorem to $S$-Willmore surfaces in $S^{n}$. He also proved that Willmore surfaces of genus 0 in $S^{4}(1)$ are $S$-Willmore surfaces and classified them. Recently, Hélein [13] constructed a Weierstrass type representation of all Willmore immersions in terms of closed one-forms. In the studies mentioned above, the most important fact about Willmore surfaces is that $\mathcal{L}[\phi]$ is invariant under conformal transformations of the ambient space. The Willmore functional is generalized to submanifolds in a Euclidean space

2000 Mathematics Subject Classification. Primary 53c42; Secondary 53c40.

Partly supported by the Grant-in-Aid for Scientific Research (No. 10640063), the Ministry of Education, Science, Sports and Culture, Japan. 
or a sphere. One is Pinkall's conformal invariant ([20]) and the other is given in Rigoli [21]. The generalized Willmore functional dealt with in this paper coincides with the latter. For a general presentation of the problem, see [26].

It is well-known that, for a submanifold $M^{m}$ in a space form, the normal curvature tensor $R^{\perp} \in C^{\infty}\left(\bigwedge^{2} T^{*} M \otimes\left(T^{\perp} M\right)^{*} \otimes T^{\perp} M\right)$ is invariant under conformal transformations of the ambient space. Therefore the functional

$$
\mathcal{R}_{q}^{\perp}[\phi]=\int_{M}\left\|R^{\perp}\right\|^{q} d v
$$

on the space of immersions $\phi: M^{m} \rightarrow \tilde{M}(c)$ is also a conformal invariant if $q=m / 2$. However, for the most part, we shall deal with the functional $\mathcal{R}_{2}^{\perp}[\phi]$, because it is the YangMills integral of the normal bundle. We shall also deal with the case that $q=1$ when $M$ is a surface. We here note that the geometric meaning of $\mathcal{L}[\phi]$ and $\mathcal{R}_{2}^{\perp}[\phi]$ for surfaces is as follows: The integrand of $\mathcal{L}[\phi]$ is equal, up to a constant factor, to the sum of the square of lengths of major and minor axes of the curvature ellipse in the normal space at each point. On the other hand, the integrand $\left\|R^{\perp}\right\|^{2}$ of $\mathcal{R} \frac{\perp}{2}[\phi]$ is equal to the square of the area encircled by the curvature ellipse up to a constant factor.

Guadalupe and Rodriguez [12] studied the integral of the normal curvature and obtained some inequalities relating the area of the surface and the integral of the square of the length of the mean curvature vector with topological invariants. Their integral of the normal curvature is different from ours. We should note that $\mathcal{R}_{1}^{\perp}[\phi]$ (resp. $\mathcal{R}_{2}^{\perp}[\phi]$ ) is the integral of the absolute value (resp. the square of the length) of the normal curvaure.

In Section 1, we give the fundamental formulas in the theory of submanifolds in a real space form. We also rewrite the corresponding formulas in terms of isothermal coordinates when the submanifold is two-dimensional.

In Section 2, we obtain the first variation formulas of $\mathcal{L}[\phi]$ and $\mathcal{R}_{q}^{\perp}[\phi]$. The EulerLagrange equation of $\mathcal{L}[\phi]$ has already known as mentioned above. However, the computation in this paper seems to be briefer than that of [22]. The Euler-Lagrange equation of $\mathcal{R}_{q}^{\perp}[\phi]$ is given in Theorem 2.7. The functional $\mathcal{R}_{2}^{\perp}[\phi]$ is a conformal invariant if the submanifold is of dimension 4 and is the Yang-Mills integral. We shall prove in Theorem 2.8 that if $\phi: M^{4} \rightarrow \tilde{M}(c)$ is an immersion of a 4-dimensional compact oriented manifold $M^{4}$ into an $n$-dimensional space form $\tilde{M}(c)$ and the normal connection is self-dual or anti-self-dual, then $\phi$ is a critical immersion of $\mathcal{R}_{2}^{\perp}[\phi]$. We should note that since $\mathcal{R}_{2}^{\perp}[\phi]$ is a functional defined on a space of immersions, the normal bundle and the induced metric vary with $\phi$.

In Section 3, we reduce the Euler-Lagrange equation of $\mathcal{R}_{q}^{\perp}[\phi](q=1$ and 2$)$ to the situation that the submanifold is a surface. The result is given in Theorem 3.1.

In Section 4, we shall study critical surfaces of $\mathcal{L}[\phi]$ and $\mathcal{R}_{2}^{\perp}[\phi]$. We give formulas relating the sum of residues of logarithmic singularities of S-Willmore points in a compact oriented Willmore surface with conformal invariants. In particular, the conformal invariant appeared in the formula (4.8) is the Willmore integral. We conclude this section by showing Theorem 4.8 that is stated as follows: 
Let $\phi: M^{2} \rightarrow S^{n}(c)$ be a minimal immersion of compact surface $M^{2}$ into $S^{n}(c)$. If $\phi$ is a critical immersion of $\mathcal{R}_{2}^{\perp}[\phi]$ and the curvature ellipses are circles, then the Gauss curvature is constant and the immersion is a standard minimal immersion of a sphere, a constant isotropic minimal immersion of a flat torus.

To complete the proof of Theorem 4.8, we need to study concircular scalar fields in Section 6.

In Section 5, we shall consider the equation satisfied by concircular scalar fields on a two-dimensional manifold $M$ as the Euler-Lagrange equation of the functional

$$
\mathcal{F}_{J}[g]=\int_{M} J(K) d v_{g},
$$

where $J$ is a function on $\boldsymbol{R}$. Moreover, we shall introduce Tashiro's work concerning concircular scalar fields.

In Section 6, by making use of elliptic functions, we classify complete two-dimensional manifolds admitting concircular scalar fields whose characteristic functions are polynomials of the scalar field which are of degree 2 or 3 . The classification is given in Theorem 6.3. The proof of Theorem 4.8 is completed by using Theorem 6.4.

The author would like to express his hearty thanks to Professor M. Okumura who introduced to him the results by Tashiro [24] explained in Section 5 and to the referee for his valuable comments.

1. Submanifolds in a space form. Let $\phi: M \rightarrow \tilde{M}$ be an immersion of an $m$ dimensional $C^{\infty}$ manifold $M$ into an $n$-dimensional Riemannian manifold $\tilde{M}$. We shall denote the Riemannian metric on $\tilde{M}$ by $\tilde{g}$ and the induced metric on $M$ by $g$. Indices $i, j, k, l$ run over the range $\{1, \ldots, m\}, \lambda, \mu, v, \kappa$ the range $\{1, \ldots, n\}$ and $u, v$ the range $\{m+1, \ldots, n\}$. The differential $d \phi$ of the map $\phi$ can be regarded as a $C^{\infty}$ section of the bundle $T^{*} M \otimes \phi^{*} T \tilde{M}$, namely $d \phi \in C^{\infty}\left(T^{*} M \otimes \phi^{*} T \tilde{M}\right)$ and, in terms of local coordinates $\left\{x^{1}, \ldots, x^{m}\right\}$ (resp. $\left.\left\{y^{1}, \ldots, y^{n}\right\}\right)$ in $M$ (resp. in $\left.\tilde{M}\right)$, it is represented as

$$
d \phi=\frac{\partial \phi^{\lambda}}{\partial x^{i}} d x^{i} \otimes \frac{\partial}{\partial y^{\lambda}}, \quad\left(y^{\lambda}=\phi^{\lambda}\left(x^{1}, \ldots, x^{m}\right)\right),
$$

where we use the so-called Einstein summantion convention. The induced metric $g$ is given by

$$
g(X, Y)=\tilde{g}((d \phi(X), d \phi(Y))
$$

for any vector fields $X$ and $Y$ tangent to $M$.

Let $N: T^{\perp} M \rightarrow \phi^{*} T \tilde{M}$ be the inclusion map of the normal bundle $T^{\perp} M$ into $\phi^{*} T \tilde{M}$. Then it is regarded as a $C^{\infty}$ section of $\operatorname{Hom}\left(T^{\perp} M, \phi^{*} T \tilde{M}\right)$. The connection on $\phi^{*} T \tilde{M}$ induced from the Levi-Civita connection on $\tilde{M}$ and the normal connection on $T^{\perp} M$ induce a connection $\nabla$ on $\operatorname{Hom}\left(T^{\perp} M, \phi^{*} T \tilde{M}\right)$. Then the Weingarten equation for $\phi$ becomes

$$
\nabla N=-d \phi \circ A,
$$


where $A \in C^{\infty}\left(T^{*} M \otimes T M \otimes\left(T^{\perp} M\right)^{*}\right)$ and, for a normal vector field $\xi, A_{\xi} \in C^{\infty}\left(T^{*} M \otimes\right.$ $T M$ ) is the shape operator correspnding to $\xi$. The relation between $A_{\xi}$ and the second fundamental form $h \in C^{\infty}\left(S^{2} T^{*} M \otimes T^{\perp} M\right)$ is given by

$$
g\left(A_{\xi} X, Y\right)=\tilde{g}(h(X, Y), \xi)
$$

for vector fields $X$ and $Y$ tangent to $M$. We shall put $H=N \circ h$, which belongs to $C^{\infty}\left(S^{2} T^{*} M, \phi^{*} T \tilde{M}\right)$. The Gauss equation is given by

$$
\nabla d \phi=H,
$$

$\nabla$ being the induced connection on the bundle $T^{*} M \otimes \phi^{*} T \tilde{M}$.

Let $\tilde{M}$ be a space form $\tilde{M}(c)$ of constant sectional curvature $c$. Then the structure equations of Gauss, Codazzi, Ricci are given, respectively, by

$$
\begin{aligned}
g(R(X, Y) Z, W)= & c\{g(X, W) g(Y, Z)-g(X, Z) g(Y, W)\} \\
& +\tilde{g}(H(X, W), H(Y, Z)-\tilde{g}(H(X, Z), H(Y, W)), \\
(\nabla h)(X, Y, Z)= & (\nabla h)(Y, X, Z), \\
R^{\perp}(X, Y) \xi= & h\left(X, A_{\xi} Y\right)-h\left(Y, A_{\xi} X\right)
\end{aligned}
$$

for $X, Y, Z, W \in T M$ and $\xi \in T^{\perp} M$ (cf. [9]), where $R^{\perp} \in C^{\infty}\left(\bigwedge^{2} T^{*} M \otimes\left(T^{\perp} M\right)^{*} \otimes\right.$ $T^{\perp} M$ ) is the normal curvature tensor. We note that $\nabla$ in (1.7) is the induced connection on $\left(T^{*} M\right)^{2} \otimes T^{\perp} M$. In the sequel, we shall use the same notation $\nabla$ for each connection induced on various vector bundles constructed from $T M, T^{\perp} M$ and $\phi^{*} T \tilde{M}$ except for the two-dimensional case, and shall not state to which vector bundle various tensors belong. From (1.6), we have formulas for Ricci tensor Ric and scalar curvature $\rho$ :

$$
\begin{gathered}
\operatorname{Ric}(X, Y)=c(m-1) g(X, Y)+m \tilde{g}(h(X, Y), \eta)-\sum_{i} \tilde{g}\left(h\left(X, X_{i}\right), h\left(Y, X_{i}\right)\right), \\
\rho=c m(m-1)+m^{2}\|\eta\|^{2}-\|H\|^{2},
\end{gathered}
$$

where $\eta$ is the mean curvature vector field defined by

$$
\eta=\frac{1}{m} \sum_{i} h\left(X_{i}, X_{i}\right)
$$

$\left\{X_{1}, \ldots, X_{m}\right\}$ being an orthonormal frame tangent to $M$.

Next, we deal with oriented $C^{\infty}$ surfaces differentiably immersed in a sphere $S^{n}(c)=$ $\left\{p \in \boldsymbol{R}^{n+1} \mid\|p\|=1 / \sqrt{c}\right\}$. Using isothermal coordinates $z=x+\sqrt{-1} y$, we write the induced metric $g$ as

$$
g=2 F(z, \bar{z})|d z|^{2},
$$

where $F$ is a positive $C^{\infty}$ function. We note that $F$ becomes real analytic if the immersion $\phi$ is minimal, has parallel mean curvature vector or makes $M$ to be a Willmore surface (cf. [11]). The area element is given by 


$$
d v=2 F d x \wedge d y=\sqrt{-1} F d z \wedge d \bar{z} .
$$

For integers $p$ and $q$, let $E^{p, q}$ be the complex line bundle over $M$ whose elements are equivalence classes of $(U, z, P, w)$, where

(a) $U$ is an open domain in $M$ and $P \in U$,

(b) $z$ is a local isothermal parameter defined in $U$ and $w \in \boldsymbol{C}$,

(c) $(U, z, P, w) \sim\left(U^{\prime}, z^{\prime}, P^{\prime}, w^{\prime}\right)$ if and only if

(i) $P=P^{\prime} \in U \cap U^{\prime}$, and

(ii) $\quad w^{\prime}=w\left(\left(\partial z / \partial z^{\prime}\right)(P)\right)^{p} \overline{\left(\left(\partial z / \partial z^{\prime}\right)(P)\right)^{q}}$.

See [8] for details. We shall sometimes use the complex conjugation $E^{p, q} \rightarrow E^{q, p}$ in our computation. If $\alpha=\bar{\alpha}$ for $\alpha \in E^{p, q}$, then it is said to be real. For instance, $F(=F(z, \bar{z}) d z \otimes$ $d \bar{z})$ is in $E^{1,1}$ and real. The Gauss curvature $K$ of $g$ is given by

$$
K=-\frac{1}{F} \partial \bar{\partial} \log F=\frac{1}{2} \Delta \log F,
$$

where $\partial=\partial / \partial z, \bar{\partial}=\partial / \partial \bar{z}$ and $\Delta=-2 F^{-1} \partial \bar{\partial}$. The metric $g$ induces Levi-Civita connection $\nabla$ on the bigraded algebra $E=\sum_{p, q} E^{p, q}$ with tensor product. The covariant differential operator $\nabla$ decomposes into $\nabla^{\prime}$ and $\nabla^{\prime \prime}$, where $\nabla^{\prime}$ (resp. $\nabla^{\prime \prime}$ ) is a differential operator of bidegree $(1,0)($ resp. $(0,1))$. The operators $\nabla^{\prime}$ and $\nabla^{\prime \prime}$ are defined by

$$
\begin{aligned}
\nabla^{\prime} \alpha & =(\partial \alpha(z, \bar{z})-p \partial \log F \cdot \alpha(z, \bar{z}))(d z)^{p+1} \otimes(d \bar{z})^{q}, \\
\nabla^{\prime \prime} \alpha & =(\bar{\partial} \alpha(z, \bar{z})-q \bar{\partial} \log F \cdot \alpha(z, \bar{z}))(d z)^{p} \otimes(d \bar{z})^{q+1}
\end{aligned}
$$

for $\alpha=\alpha(z, \bar{z})(d z)^{p} \otimes(d \bar{z})^{q} \in C^{\infty}\left(E^{p, q}\right)$. In particular, we have $\nabla^{\prime} F=0=\nabla^{\prime \prime} F$. For the Ricci identity, we have

$$
\left[\nabla^{\prime}, \nabla^{\prime \prime}\right] \alpha=(q-p) K F \otimes \alpha .
$$

All higher order derivatives of $\phi$ will be considered as functions with values in $\boldsymbol{C}^{n+1}=$ $\boldsymbol{R}^{n+1} \otimes_{\boldsymbol{R}} \boldsymbol{C}$. Let the symmetric product $\mathbf{a}=\left(a_{1}, \ldots, a_{n+1}\right)$ and $\mathbf{b}=\left(b_{1}, \ldots, b_{n+1}\right)$ in $\boldsymbol{C}^{n+1}$ be defined by

$$
\langle\mathbf{a}, \mathbf{b}\rangle=\sum_{h=1}^{n+1} a_{h} b_{h} .
$$

Then the Hermitian product on $\boldsymbol{C}^{n+1}$ is given by $\langle\mathbf{a}, \overline{\mathbf{b}}\rangle$. The norm of $\alpha \in E^{p, q} \otimes \boldsymbol{C}^{n+1}$ is defined as

$$
|\alpha|^{2}=F^{-(p+q)}\langle\alpha, \bar{\alpha}\rangle
$$

We immediately have

$$
\left\langle\nabla^{\prime} \phi, \nabla^{\prime} \phi\right\rangle=0, \quad\left\langle\nabla^{\prime \prime} \phi, \nabla^{\prime \prime} \phi\right\rangle=0, \quad\left\langle\nabla^{\prime} \phi, \nabla^{\prime \prime} \phi\right\rangle=F .
$$

Let $x^{1}=x$ and $x^{2}=y$. We put $H_{i j}=H\left(\partial / \partial x^{i}, \partial / \partial x^{j}\right)$, where $H$ is the second fundamental form of the immersion $\phi: M \rightarrow S^{n}(c)$. If we consider $H_{i j}$ as a vector in $\boldsymbol{C}^{n+1}$, then we see that the Gauss equation (1.5) becomes 


$$
\nabla^{\prime 2} \phi=\frac{1}{4}\left(H_{11}-H_{22}-2 \sqrt{-1} H_{12}\right)(d z)^{2} .
$$

The right hand side is a vector normal to $M$ in $S^{n}(c)$, which we shall denote by $\gamma$. The mean curvature vector field $\eta$ satisfies

$$
\eta=c \phi-\frac{1}{2} \Delta \phi,
$$

because $\Delta \phi=-(2 / F) \nabla^{\prime} \nabla^{\prime \prime} \phi$. Taking a local orthonormal cross sections $\left\{N_{3}, \ldots, N_{n}\right\}$ in $T^{\perp} M$ and regarding them as $\boldsymbol{C}^{n+1}$-valued functions, we have

$$
\left(\partial N_{u}+\eta^{u} \partial \phi+\frac{1}{F} \gamma^{u} \bar{\partial} \phi\right) d z=\omega_{u}^{v} N_{v},
$$

where $\eta=\eta^{v} N_{v}, \gamma=\gamma^{v} N_{v}$ and $\omega_{u}^{v}$ is the components of the normal connection extended to the complexification $C T^{\perp} M$ of the normal bundle. For $\xi \in C^{\infty}\left(E^{p, q} \otimes C T^{\perp} M\right)$, we may define the covariant differentiation of $\xi$ by

$$
{ }^{\prime} \nabla^{\perp} \xi=\left({ }^{\prime} \nabla \xi^{u}+\omega_{v}^{u} \xi^{v}\right) N_{u}, \quad{ }^{\prime \prime} \nabla^{\perp} \xi=\left({ }^{\prime \prime} \nabla \xi^{u}+\bar{\omega}_{v}^{u} \xi^{v}\right) N_{u},
$$

where $\xi=\xi^{u} N_{u}=\xi^{u}(z, \bar{z})(d z)^{p} \otimes(d \bar{z})^{q} N_{u}$. Then the Weingarten equation (1.3) becomes

$$
\nabla^{\prime} \xi-{ }^{\prime} \nabla^{\perp} \xi=-\langle\xi, \eta\rangle \nabla^{\prime} \phi-\frac{1}{F}\langle\xi, \gamma\rangle \nabla^{\prime \prime} \phi,
$$

in virtue of (1.21).

The structure equation (1.6) through (1.8) of Gauss, Codazzi and Ricci are the following:

$$
\begin{aligned}
K & =c+\|\eta\|^{2}-|\gamma|^{2}, \\
{ }^{\prime \prime} \nabla^{\perp} \gamma & =F^{\prime} \nabla^{\perp} \eta, \\
\mathfrak{R}^{\perp} \xi & =\frac{1}{F}\{\langle\xi, \bar{\gamma}\rangle \gamma-\langle\xi, \gamma\rangle \bar{\gamma}\},
\end{aligned}
$$

for $\xi \in C^{\infty}\left(E^{p, q} \otimes C T^{\perp} M\right)$, where

$$
\mathfrak{R}^{\perp}=\nabla^{\prime} \bar{\omega}-\nabla^{\prime \prime} \omega+[\omega, \bar{\omega}] \in C^{\infty}\left(E^{1,1} \otimes \operatorname{Hom}\left(\boldsymbol{C} T^{\perp} M, \boldsymbol{C} T^{\perp} M\right)\right) .
$$

We note that the components of $\Re^{\perp}$ are given by

$$
\mathfrak{R}^{\perp}{ }_{u}{ }^{v}=\frac{\sqrt{-1}}{2} R^{\perp} 12 u^{v} d z \otimes d \bar{z},
$$

and hence it is pure imaginary. We finally note that Ricci identity for $\xi \in C^{\infty}\left(E^{p, q} \otimes C T^{\perp} M\right)$ is

$$
\left[{ }^{\prime} \nabla^{\perp},{ }^{\prime \prime} \nabla^{\perp}\right] \xi=(q-p) K F \otimes \xi+\mathfrak{R}^{\perp} \xi .
$$

2. Variation of the length of normal curvature tensor. Let $\phi$ be an immersion of an oriented $m$-dimensional manifold $M$ into an $n$-dimensional Riemannian manifold $\tilde{M}$. We shall assume that $m \geq 2$. 
DEFINITION. By a compactly supported variation of $\phi$, we mean a $C^{\infty}$ map $\Phi$ : $(-\varepsilon, \varepsilon) \times M \rightarrow \tilde{M}$, (where $\varepsilon>0)$, such that

(a) each map $\phi_{t}=\Phi(t, \cdot): M \rightarrow \tilde{M}$ is an immersion and $\phi=\phi_{0}$,

(b) the closure of the set $\left\{p \in M \mid \phi_{t}(p) \neq \phi(p)\right.$ for some $\left.t \in(-\varepsilon, \varepsilon)\right\}$ is compact.

The variation vector $V$ of $\Phi$ is a vector field along $\phi$ which is defined by

$$
V=\left.d \Phi\left(\frac{\partial}{\partial t}\right)\right|_{t=0} .
$$

Thus, if we put $W=d \Phi(\partial / \partial t)$, then $W(0, p)=V(p)$ for every $p \in M$. We decompose $W$ into the tangential and normal components:

$$
W=d \phi_{t}(T)+N_{t} \zeta
$$

where $N_{t}$ is the inclusion map of the normal bundle $\left(T^{\perp} M\right)_{t}$ into $\phi_{t}^{*} T \tilde{M}$ with respect to the immersion $\phi_{t}$. We note that $T$ and $\zeta$ depend on $t$, but we omit $t$ from them. On $(-\varepsilon, \varepsilon) \times M$, we define an operator $\delta_{t}$ by

$$
\delta_{t} f=\frac{\partial f}{\partial t}, \quad \delta_{t}\left(\frac{\partial}{\partial y^{\lambda}}\right)_{\Phi}=W^{\mu} \tilde{\Gamma}_{\mu}{ }^{\nu} \lambda\left(\frac{\partial}{\partial y^{v}}\right)_{\Phi}, \quad \delta_{t} \frac{\partial}{\partial x^{i}}=0
$$

for every $\lambda$ and $i$, where $f$ is a $C^{\infty}$ function on $(-\varepsilon, \varepsilon) \times M, \tilde{\Gamma}_{\mu}{ }^{\nu} \lambda$ are Christoffel's symbols of the Levi-Civita connection of $\tilde{M}$ and $\left(\partial / \partial y^{\lambda}\right)_{\Phi}$ is the natural local frame in $\Phi^{*} T \tilde{M}$. We extend $\delta_{t}$ as a derivation to the tensor bundle $\sum T_{s}^{r}(M) \otimes \Phi^{*} T_{q}^{p}(\tilde{M})$.

LEMMA 2.1. Let $g_{t}$ be the induced metric $\phi_{t}^{*} \tilde{g}$ on $\{t\} \times M$ for each $t \in(-\varepsilon, \varepsilon)$. Let $\mathscr{L}_{T}$ denote the Lie derivative with respect to $T$ and $\left(H_{t}\right)_{\zeta}$ be defined by $\left(H_{t}\right)_{\zeta}(X, Y)=$ $\tilde{g}\left(H_{t}(X, Y), N_{t} \zeta\right)$ for $X, Y \in T M$, where $H_{t}$ is the second fundamental form of the immersion $\phi_{t}$. Then we have

$$
\delta_{t} g_{t}=\mathscr{L}_{T} g_{t}-2\left(H_{t}\right)_{\zeta}
$$

PROOF. We first note that

$$
\delta_{t} \tilde{g}=0,
$$

since

$$
\begin{aligned}
\delta_{t}\left(\tilde{g}_{\lambda \mu} d y^{\lambda} \otimes d y^{\mu}\right)= & W^{\nu} \frac{\partial}{\partial y^{\nu}} \tilde{g}_{\lambda \mu} d y^{\lambda} \otimes d y^{\mu} \\
& -\tilde{g}_{\lambda \mu} W^{\kappa} \tilde{\Gamma}_{\kappa}^{\lambda}{ }_{\nu} d y^{\nu} \otimes d y^{\mu}-\tilde{g}_{\lambda \mu} W^{\kappa} \tilde{\Gamma}_{\kappa}{ }^{\mu}{ }_{\nu} d y^{\lambda} \otimes d y^{\nu} \\
= & W^{\nu} \nabla_{\nu} \tilde{g}_{\lambda \mu} d y^{\lambda} \otimes d y^{\mu}=0 .
\end{aligned}
$$

It follows from (2.4) that

$$
\begin{aligned}
\left(\delta_{t} g_{t}\right)(X, Y) & =\delta_{t}\left(g_{t}(X, Y)\right)=\delta_{t}\left(\tilde{g}\left(d \phi_{t}(X), d \phi_{t}(Y)\right)\right) \\
& =\tilde{g}\left(\left(\delta_{t} d \phi_{t}\right)(X), d \phi_{t}(Y)\right)+\tilde{g}\left(d \phi_{t}(X),\left(\delta_{t} d \phi_{t}\right)(Y)\right)
\end{aligned}
$$


for every $X, Y \in T M$. Since

$$
\begin{aligned}
\delta_{t} d \phi_{t} & =\delta_{t}\left(\frac{\partial \Phi^{\lambda}}{\partial x^{i}} d x^{i} \otimes\left(\frac{\partial}{\partial y^{\lambda}}\right)_{\Phi}\right) \\
& =\left(\frac{\partial W^{\lambda}}{\partial x^{i}}+W^{\nu} \tilde{\Gamma}_{\nu}^{\lambda} \mu \frac{\partial \Phi^{\mu}}{\partial x^{i}}\right) d x^{i} \otimes\left(\frac{\partial}{\partial y^{\lambda}}\right)_{\Phi} \\
& =\nabla W
\end{aligned}
$$

we have, from (1.5),

$$
\begin{aligned}
\left(\delta_{t} g_{t}\right)(X, Y)= & \tilde{g}\left(\nabla_{X} W, d \phi_{t}(Y)\right)+\tilde{g}\left(d \phi_{t}(X), \nabla_{Y} W\right) \\
= & X \cdot \tilde{g}\left(W, d \phi_{t}(Y)\right)-\tilde{g}\left(W,\left(\nabla_{X}^{t} d \phi_{t}\right)(Y)\right)-\tilde{g}\left(W, d \phi_{t}\left(\nabla_{X}^{t} Y\right)\right) \\
& +Y \cdot \tilde{g}\left(d \phi_{t}(X), W\right)-\tilde{g}\left(\left(\nabla_{Y}^{t} d \phi_{t}\right)(X), W\right)-\tilde{g}\left(d \phi_{t}\left(\nabla_{Y}^{t} X\right), W\right) \\
= & X \cdot g_{t}(T, Y)-g_{t}\left(T, \nabla_{X}^{t} Y\right)+Y \cdot g_{t}(X, T)-g_{t}\left(\nabla_{Y}^{t} X, T\right) \\
& -\tilde{g}\left(N_{t} \zeta, H_{t}(X, Y)\right)-\tilde{g}\left(H_{t}(Y, X), N_{t} \zeta\right) \\
= & \left(\mathscr{L}_{T} g_{t}\right)(X, Y)-2\left(H_{t}\right)_{\zeta}(X, Y),
\end{aligned}
$$

where $\nabla^{t}$ is the induced connection on the bundle $T^{*} M \otimes \phi_{t}^{*} \tilde{M}$ over $\{t\} \times M$.

LEMMA 2.2. Let $g_{t}^{-1}$ be the inverse matrix of the metric $g_{t}$ and $d v_{t}$ be the volume form on $M$ with respect to $g_{t}$. Then we have

$$
\begin{aligned}
\delta_{t} g_{t}^{-1} & =-g_{t}^{-1}\left\{\mathscr{L}_{T} g_{t}-2\left(H_{t}\right)_{\zeta}\right\} g_{t}^{-1}, \\
\delta_{t} d v_{t} & =\{\operatorname{div} T-m \tilde{g}(\eta, \zeta)\} d v_{t},
\end{aligned}
$$

$\operatorname{div} T$ denoting the divergence of the vector field $T$.

Proof. Since

$$
0=\delta_{t}\left(g_{t} g_{t}^{-1}\right)=\left(\delta_{t} g_{t}\right) g_{t}^{-1}+g_{t}\left(\delta_{t} g_{t}^{-1}\right),
$$

substituting (2.3) into the first term, we obtain (2.5). We denote the determinant of $g_{t}$ by $\mathfrak{g}_{t}$ and $(i, k)$-cofactor by $\triangle_{i k}$. Then $\partial \sqrt{\mathfrak{g}_{t}} / \partial t=\left(\partial \mathfrak{g}_{t} / \partial t\right) / 2 \sqrt{\mathfrak{g}_{t}}$ and

$$
\begin{aligned}
\partial \mathfrak{g}_{t} / \partial t & =\sum_{k=1}^{m}\left\{\frac{\partial\left(g_{t}\right)_{1 k}}{\partial t} \Delta_{1 k}+\frac{\partial\left(g_{t}\right)_{2 k}}{\partial t} \Delta_{2 k}+\cdots+\frac{\partial\left(g_{t}\right)_{m k}}{\partial t} \Delta_{m k}\right\} \\
& =\sum_{k} \sum_{i}\left(\delta_{t} g_{t}\right)_{i k}\left(g_{t}\right)^{i k} \mathfrak{g}_{t}=\mathfrak{g}_{t}\left(\mathscr{L}_{T} g_{t}-2\left(H_{t}\right)_{\zeta}\right)_{i k}\left(g_{t}\right)^{i k} \\
& =2\{\operatorname{div} T-m \tilde{g}(\eta, \zeta)\} \mathfrak{g}_{t} .
\end{aligned}
$$

Thus we have (2.6).

LEMMA 2.3. The variation $\delta_{t} H_{t}$ of the second fundamental form $H_{t}$ is given by

$$
\left(\delta_{t} H_{t}\right)(X, Y)=\left(\nabla^{t} \nabla W\right)(X, Y)+\tilde{R}\left(W, d \phi_{t}(X)\right) d \phi_{t}(Y)-d \phi_{t}\left(\left(\delta_{t} \Gamma\right)(X, Y)\right)
$$

for every $X, Y \in T M$, where $\left.\delta_{t} \Gamma=\left(\partial \Gamma_{j}{ }^{i}{ }_{k}(t)\right) / \partial t\right) d x^{j} \otimes d x^{k} \otimes \partial / \partial x^{i}, \Gamma_{j}{ }^{i}{ }_{k}(t)$ being Christoffel's symbols of the Levi-Civita connection of $g_{t}$. 
ProOF. From (1.5), we have

$$
\left(\delta_{t} H_{t}\right)(X, Y)=\left(\delta_{t} \nabla^{t} d \phi_{t}\right)(X, Y)
$$

for $X, Y \in T M$, where we note that

$$
\nabla^{t} d \phi_{t}=\left(\frac{\partial^{2}}{\partial x^{i} \partial x^{j}} \Phi^{\lambda}+\frac{\partial \Phi^{\nu}}{\partial x^{i}} \tilde{\Gamma}_{\nu}^{\lambda}{ }_{\mu} \frac{\partial \Phi^{\mu}}{\partial x^{j}}-\Gamma_{i}^{k}{ }_{j}(t) \frac{\partial \Phi^{\lambda}}{\partial x^{k}}\right) d x^{i} \otimes d x^{j} \otimes\left(\partial / \partial y^{\lambda}\right)_{\Phi}
$$

Therefore, using the Ricci formula

$$
\left[\delta_{t}, \nabla^{t}\right] d \phi_{t}=\tilde{R}\left(W, d \phi_{t}(X)\right) d \phi_{t}(Y)-d \phi_{t}\left(\left(\delta_{t} \Gamma\right)(X, Y)\right)
$$

and $\delta_{t} d \phi_{t}=\nabla W$, we obtain (2.7).

REMARK. In our later computation, we shall take an inner product of $\delta_{t} H_{t}$ with normal vectors and so we need not compute $\delta_{t} \Gamma$. Here, we only note that $\delta_{t} \Gamma$ is a tensor field given by

$$
2 g_{t}\left(\left(\delta_{t} \Gamma\right)(X, Y), Z\right)=\left(\nabla^{t} k\right)(X, Y, Z)+\left(\nabla^{t} k\right)(Y, Z, X)-\left(\nabla^{t} k\right)(Z, X, Y),
$$

where $k=\mathscr{L}_{T} g_{t}-2\left(H_{t}\right)_{\zeta}$. This will be necessary for the computation of the second variation formula.

We next compute the first and second terms of the right hand side of (2.7). Hereafter, we assume that $\tilde{M}$ is a Riemannian manifold $\tilde{M}(c)$ of constant sectional curvature $c$.

LEMMA 2.4. For every $X, Y \in T M$, we have

$$
\begin{aligned}
\tilde{R}\left(W, d \phi_{t}(X)\right) d \phi_{t}(Y) \equiv c g_{t}(X, Y) N_{t} \zeta \quad \bmod d \phi_{t}(T M) \\
\left(\nabla^{t} \nabla W\right)(X, Y) \equiv N_{t}\left\{\left(\nabla^{t} h_{t}\right)(X, Y, T)+h_{t}\left(\nabla_{X}^{t} T, Y\right)+h_{t}\left(X, \nabla_{Y}^{t} T\right)\right. \\
\left.+\left(\nabla^{t} \nabla^{t} \zeta\right)(X, Y)-h_{t}\left(X, A_{\zeta}^{t} Y\right)\right\} \quad \bmod d \phi_{t}(T M) .
\end{aligned}
$$

ProOf. Since $\tilde{M}=\tilde{M}(c)$, we have

$$
\begin{aligned}
\tilde{R}\left(W, d \phi_{t}(X)\right) d \phi_{t}(Y) & =c \tilde{g}\left(d \phi_{t}(X), d \phi_{t}(Y)\right) W-\tilde{g}\left(W, d \phi_{t}(Y)\right) d \phi_{t}(X) \\
& \equiv c g_{t}(X, Y) N_{t} \zeta \quad \bmod d \phi_{t}(T M)
\end{aligned}
$$

Equation (2.9) is proved as follows:

$$
\begin{aligned}
\nabla_{Y} W & =\nabla_{Y}^{t}\left(d \phi_{t}(T)+N_{t} \zeta\right) \\
& =H_{t}(Y, T)+d \phi_{t}\left(\nabla_{Y}^{t} T\right)-d \phi_{t}\left(A_{\zeta}^{t} Y\right)+N_{t} \nabla_{Y}^{t} \zeta .
\end{aligned}
$$


Therefore, if $X \in T_{p} M$ and $Y$ is a vector field on $M$ such that $\nabla_{X} Y=0$ at $p$, then

$$
\begin{aligned}
\left(\nabla^{t} \nabla W\right)(X, Y)= & \nabla_{X}^{t} \nabla_{Y} W \\
= & \nabla_{X}^{t}\left\{N_{t} h_{t}(Y, T)+d \phi_{t}\left(\nabla_{Y}^{t} T\right)-d \phi_{t}\left(A_{\zeta}^{t} Y\right)+N_{t} \nabla_{Y}^{t} \zeta\right\} \\
\equiv & N_{t}\left(\nabla^{t} h_{t}\right)(X, Y, T)+N_{t} h_{t}\left(Y, \nabla_{X}^{t} T\right) \\
& +H_{t}\left(X, \nabla_{Y}^{t} T\right)-H_{t}\left(X, A_{\zeta}^{t} Y\right)+N_{t} \nabla_{X}^{t} \nabla_{Y}^{t} \zeta \\
= & N_{t}\left\{\left(\nabla^{t} h_{t}\right)(X, Y, T)+h_{t}\left(X, \nabla_{Y}^{t} T\right)+h_{t}\left(Y, \nabla_{X}^{t} T\right)\right. \\
& \left.\quad-h_{t}\left(X, A_{\zeta}^{t} Y\right)+\left(\nabla^{t} \nabla^{t} \zeta\right)(X, Y)\right\} \quad \bmod d \phi_{t}\left(T_{p} M\right),
\end{aligned}
$$

where $A_{\zeta}^{t}$ is the shape operator of $\phi_{t}$ with respect to $\zeta$.

It follows from (2.7) through (2.9) that

$$
\begin{aligned}
(\delta H)(X, Y) \equiv N\{ & (\nabla \nabla \zeta)(X, Y)+c g(X, Y) \zeta+(\nabla h)(T, X, Y) \\
& \left.-h\left(X, A_{\zeta} Y\right)+h\left(\nabla_{X} T, Y\right)+h\left(X, \nabla_{Y} T\right)\right\} \quad \bmod d \phi(T M),
\end{aligned}
$$

where we have put $\delta=\left.\delta_{t}\right|_{t=0}$ and so on. For the mean curvature vector $\eta$, we have

$$
\delta(N \eta) \equiv N\left\{\frac{1}{m}\left(-\Delta \zeta+S^{\perp} \zeta\right)+c \zeta+\nabla_{T} \eta\right\} \quad \bmod d \phi(T M),
$$

$S^{\perp}$ being the symmetric transformation $T^{\perp} M \rightarrow T^{\perp} M$ defined by

$$
\tilde{g}\left(S^{\perp} \xi, \xi^{\prime}\right)=\operatorname{trace}\left(A_{\xi} A_{\xi^{\prime}}\right) .
$$

Here we take an orthonormal local frame field $\left\{N_{u}\right\}$ in $T^{\perp} M$. The equation (2.11) is proved as follows:

$$
\begin{aligned}
\delta(N \eta)= & \frac{1}{m} \delta\left\{g^{i j} H_{i j}^{\lambda}\left(\frac{\partial}{\partial y^{\lambda}}\right)_{\phi}\right\} \\
= & \frac{1}{m}\left\{\left(\delta g^{-1}\right)^{i j} H_{i j}^{\lambda}\left(\frac{\partial}{\partial y^{\lambda}}\right)_{\phi}+g^{i j}(\delta H)_{i j}^{\lambda}\left(\frac{\partial}{\partial y^{\lambda}}\right)_{\phi}\right\} \\
\equiv & \frac{1}{m}\left\{-\left(\nabla^{i} T^{j}+\nabla^{j} T^{i}\right)+2 h^{i j}{ }_{u} \zeta^{u}\right\} H_{i j}^{\lambda}\left(\frac{\partial}{\partial y^{\lambda}}\right)_{\phi} \\
& +\frac{1}{m} N\left(-\Delta \zeta-S^{\perp} \zeta+c m \zeta+m \nabla_{T} \eta\right)+\frac{2}{m}\left(\nabla^{i} T^{j}\right) H_{i j}{ }^{\lambda}\left(\frac{\partial}{\partial y^{\lambda}}\right)_{\phi} \\
= & \frac{1}{m} N\left(-\Delta \zeta+S^{\perp} \zeta\right)+c N \zeta+N \nabla_{T} \eta \bmod d \phi(T M),
\end{aligned}
$$

because of (2.5) and (2.10). Let $\left\{X_{i}\right\}_{i=1, \ldots, m}$ be an orthonormal base in $T_{p} M$.

LEMMA 2.5. Let $S$ be the symmetric transformation of $T M$ defined by $g(S X, Y)=$ $\sum_{i} \tilde{g}\left(H\left(X, X_{i}\right), H\left(Y, X_{i}\right)\right)$. Then the variation of the length of the second fundamental form 
and the mean curvature vector are given by

$$
\begin{aligned}
\delta\|H\|^{2}= & 2 \sum_{i, j} \tilde{g}\left(H\left(X_{i}, X_{j}\right),(\nabla \nabla \zeta)\left(X_{i}, X_{j}\right)\right)+2 \sum_{i} H_{\zeta}\left(S X_{i}, X_{i}\right) \\
& +2 m c \tilde{g}(\eta, \zeta)+d\|H\|^{2}(T), \\
\delta\|\eta\|^{2}= & -\frac{2}{m} \tilde{g}(\Delta \zeta, \eta)+\frac{2}{m} \tilde{g}\left(S^{\perp} \zeta, \eta\right)+2 c \tilde{g}(\zeta, \eta)+d\|\eta\|^{2}(T),
\end{aligned}
$$

respectively.

PROOF. Since

$$
\|H\|^{2}=H_{i j}{ }^{\lambda} H_{k l}{ }^{\mu} g^{i k} g^{j l} \tilde{g}_{\lambda \mu} \quad \text { and } \quad\|\eta\|^{2}=\tilde{g}(N \eta, N \eta),
$$

equations (2.12) and (2.13) are derived from (2.4), (2.5), (2.10) and (2.11) by a routine calculation.

Next, we shall compute the variation of the length of the tensor field $L$, which we define by $L=h-\eta g$, and the normal curvature tensor $R^{\perp}$. We note that $N L$ and $R^{\perp}$ are conformally invariant, that is, $N^{*} L^{*}=N L$ and $\left(R^{\perp}\right)^{*}=R^{\perp}$ under the change $\tilde{g}^{*}=e^{2 f} \tilde{g}$. This fact is well-known. However, for reader's convenience, we give the proof. By a straightforward computation, we have

$$
H^{*}(X, Y)=H(X, Y)+g(X, Y) \xi_{f},
$$

where $\xi_{f}$ is the normal component of the gradient vector of $f$. Hence the mean curvature vector satisfies

$$
N^{*} \eta^{*}=e^{-2 f}\left(N \eta+\xi_{f}\right)
$$

from which it follows that

$$
\begin{aligned}
N^{*} L^{*}(X, Y) & =H^{*}(X, Y)-g^{*}(X, Y) N^{*} \eta^{*} \\
& =H(X, Y)-g(X, Y) N \eta \\
& =N L(X, Y)
\end{aligned}
$$

Define $L_{\xi}$ by

$$
g\left(L_{\xi} X, Y\right)=\tilde{g}(L(X, Y), \xi) .
$$

Then we can easily show that

$$
\begin{aligned}
L_{\xi}^{*} X & =A_{\xi}^{*} X-\tilde{g}^{*}\left(\eta^{*}, \xi\right) X \\
& =A_{\xi} X+\tilde{g}\left(\xi_{f}, \xi\right) X-\tilde{g}\left(\eta+\xi_{f}, \xi\right) X \\
& =L_{\xi} X
\end{aligned}
$$

Since

$$
\begin{aligned}
N R^{\perp}(X, Y) \xi & =H\left(X, A_{\xi} Y\right)-H\left(Y, A_{\xi} X\right) \\
& =N L\left(X, L_{\xi} Y\right)-N L\left(Y, L_{\xi} X\right)
\end{aligned}
$$


we have $\left(R^{\perp}\right)^{*}=R^{\perp}$. Since

$$
\|L\|^{2}=\|H\|^{2}-m\|\eta\|^{2},
$$

equations (2.12) and (2.13) imply that

$$
\begin{aligned}
\delta\|L\|^{2}= & 2 \sum_{i, j} \tilde{g}\left(L\left(X_{i}, X_{j}\right),(\nabla \nabla \zeta)\left(X_{i}, X_{j}\right)\right)+2 \sum_{i} \tilde{g}\left(h\left(S X_{i}, X_{i}\right), \zeta\right) \\
& -2 \tilde{g}\left(S^{\perp} \eta, \zeta\right)+d\|L\|^{2}(T) .
\end{aligned}
$$

Therefore we have the following result which was obtained in [21] and [22].

THEOREM 2.6 ([21, 22, 26]). The Euler-Lagrange equation of the conformally invariant functional

$$
\mathcal{L}[\phi]=\int_{M}\|L\|^{m} d v
$$

is

$$
\square\left(\|L\|^{m-2} L\right)-\|L\|^{m-2}\left\{(m-1) Q^{\perp} \eta-\sum_{i, j} \operatorname{Ric}\left(X_{i}, X_{j}\right) L\left(X_{i}, X_{j}\right)\right\}=0,
$$

where $Q^{\perp}: T^{\perp} M \rightarrow T^{\perp} M$ is the symmetric transformation defined by

$$
\tilde{g}\left(Q^{\perp} \xi, \xi^{\prime}\right)=\sum \tilde{g}\left(L\left(X_{i}, X_{j}\right), \xi\right) \tilde{g}\left(L\left(X_{i}, X_{j}\right), \xi^{\prime}\right)
$$

and $\square B=-\left(\nabla^{i} \nabla^{j} B_{i j}{ }^{u}\right) N_{u}$ for any section $B$ of $T^{*} M \otimes T^{*} M \otimes T^{\perp} M$.

PROOF. We see from (2.6) that

$$
\begin{aligned}
\left.\frac{d}{d t} \mathcal{L}\left[\phi_{t}\right]\right|_{t=0} & =\int \delta\left(\|L\|^{m} d v\right) \\
& =\int\left\{\frac{m}{2}\|L\|^{m-2} \delta\|L\|^{2}+\|L\|^{m}(\operatorname{div} T-m \tilde{g}(\eta, \zeta))\right\} d v .
\end{aligned}
$$

Using (2.15), we have

$$
\begin{aligned}
\frac{m}{2}\|L\|^{m-2} \delta\|L\|^{2}=m\|L\|^{m-2}\{ & \sum_{i, j} \tilde{g}\left(L\left(X_{i}, X_{j}\right),(\nabla \nabla \zeta)\left(X_{i}, X_{j}\right)\right) \\
& \left.+\sum_{i} \tilde{g}\left(h\left(S X_{i}, X_{i}\right), \zeta\right)-\tilde{g}\left(S^{\perp} \eta, \zeta\right)+\frac{1}{2} d\|L\|^{2}(T)\right\} .
\end{aligned}
$$


Hence

$$
\begin{aligned}
& \frac{m}{2}\|L\|^{m-2} \delta\|L\|^{2}+\|L\|^{m}(\operatorname{div} T-m \tilde{g}(\eta, \zeta)) \\
&= m \sum \tilde{g}\left(\|L\|^{m-2} L\left(X_{i}, X_{j}\right), \nabla \nabla \zeta\left(X_{i}, X_{j}\right)\right) \\
&+m\|L\|^{m-2}\left\{\tilde{g}\left(\sum h\left(S X_{i}, X_{i}\right)-S^{\perp} \eta-\|L\|^{2} \eta, \zeta\right)\right\} \\
&+\frac{m}{2}\|L\|^{m-2} d\|L\|^{2}(T)+\|L\|^{m} \operatorname{div} T \\
&= m \sum \tilde{g}\left(\|L\|^{m-2} L\left(X_{i}, X_{j}\right), \nabla \nabla \zeta\left(X_{i}, X_{j}\right)\right) \\
&+m\|L\|^{m-2} \tilde{g}\left(-\sum \operatorname{Ric}\left(X_{i}, X_{j}\right) L\left(X_{i}, X_{j}\right)+(m-1) Q^{\perp} \eta, \zeta\right)+\operatorname{div}\left(\|L\|^{m} T\right)
\end{aligned}
$$

where we have used

$$
\begin{aligned}
S^{\perp} \eta & =Q^{\perp} \eta+m\|\eta\|^{2} \eta, \\
g(S X, Y) & =-\operatorname{Ric}(X, Y)+c(m-1) g(X, Y)+m \tilde{g}(h(X, Y), \eta) .
\end{aligned}
$$

Integrating by parts and using Stokes' formura, we obtain (2.16).

Thus if the Ricci tensor is proportional to the metric tensor, then (2.16) reduces to

$$
\square\left(\|L\|^{m-2} L\right)-(m-1)\|L\|^{m-2} Q^{\perp} \eta=0 .
$$

In particular, we have the following result obtained in [21, 25].

COROLLARY. If $m=2$, then (2.16) reduces to

$$
\Delta \eta-Q^{\perp} \eta=0 .
$$

Proof. We have only to show $\square L=(m-1) \Delta \eta$. We can easily show that by using the Codazzi equation (1.7).

DEFINITION. Willmore surface is a surface satisfying (2.17) immersed in a space form.

Let us consider a variational problem for another conformal invariant $R^{\perp}$. We shall compute the Euler-Lagrange equation for the functional

$$
\mathcal{R}_{q}^{\perp}[\phi]=\int\left\|R^{\perp}\right\|^{q} d v .
$$

We note that if $q=m / 2$, then $\mathcal{R}_{m / 2}^{\perp}[\phi]$ is a conformal invariant. However we are also interested in the case $q=2$ for any dimension $m$, because the right hand side of the definition of $\mathcal{R}_{2}^{\perp}[\phi]$ is a Yang-Mills integral.

Here we explain the geometric meaning of $\left\|R^{\perp}\right\|$ in the case that $q=1$ and $m=2$ (cf. [12]). For arbitrarily fixed point $p \in M$, the curvature ellipse $E_{p}$ at $p$ is defined as the set $\left\{h(X, X) \mid X \in T_{p} M,\|X\|=1\right\}$. This is an ellipse lying on the plane $\Pi_{p}$ which pass through $\eta$ and is spanned by the normal vectors $\mathbf{a}=\left(h_{11}-h_{22}\right) / 4 F$ and $\mathbf{b}=h_{12} / 2 F$ in the normal space $T_{p}{ }^{\perp} M$. We easily see that $4|\gamma|^{2}\left(=2\|L\|^{2}\right)$ is equal to $4\left(\|\mathbf{a}\|^{2}+\|\mathbf{b}\|^{2}\right)$ and hence is equal to the sum of the square of lengths of major and minor axes. The square of the area 
surrounded by $E_{p}$ in $\Pi_{p}$ is equal to $\pi^{2}\left(\|\mathbf{a}\|^{2}\|\mathbf{b}\|^{2}-\langle\mathbf{a}, \mathbf{b}\rangle^{2}\right)$. It follows that it is equal to $\pi^{2}\left(|\gamma|^{4}-|\langle\gamma, \gamma\rangle|^{2}\right) / 4$ at $p$. Since $\left|\mathfrak{R}^{\perp}\right|^{2}=F^{-2} \sum_{u, v} \mathfrak{R}^{\perp}{ }_{u}{\overline{\mathfrak{R}^{\perp}}}_{u}$, we see from (1.26) that $(\text { area })^{2}=\pi^{2}\left|\Re^{\perp}\right|^{2} / 8=\pi^{2}\left\|R^{\perp}\right\|^{2} / 16$ at $p$.

THEOREM 2.7. Let $C \in C^{\infty}\left(T^{*} M \otimes T^{*} M \otimes T^{\perp} M\right)$ be defined by

$$
C(X, Y)=\sum_{i=1}^{m} R^{\perp}\left(Y, X_{i}\right) h\left(X, X_{i}\right) .
$$

If $q \geq 2$, then the Euler-Lagrange equation of the functional

$$
\mathcal{R}_{q}^{\perp}[\phi]=\int\left\|R^{\perp}\right\|^{q} d v
$$

is given by

$$
\square\left(\left\|R^{\perp}\right\|^{q-2} C\right)-\frac{1}{2}\left\|R^{\perp}\right\|^{q-2}\left\{\sum_{i, j} P\left(X_{i}, X_{j}\right) h\left(X_{i}, X_{j}\right)-\frac{m}{2 q}\left\|R^{\perp}\right\|^{2} \eta\right\}=0,
$$

where $P$ is defined by

$$
P(X, Y)=-\sum \operatorname{trace}\left(R^{\perp}\left(X, X_{i}\right) R^{\perp}\left(Y, X_{i}\right)\right) .
$$

In particular, if $q=2$, then (2.18) becomes

$$
\square C-\frac{1}{2}\left\{\sum_{i, j} P\left(X_{i}, X_{j}\right) h\left(X_{i}, X_{j}\right)-\frac{m}{4}\left\|R^{\perp}\right\|^{2} \eta\right\}=0 .
$$

If $q=1$ and $R^{\perp} \neq 0$ anywhere on $M$, then we have

$$
\square\left(\left\|R^{\perp}\right\|^{-1} C\right)-\frac{1}{2}\left\|R^{\perp}\right\|^{-1}\left\{\sum_{i, j} P\left(X_{i}, X_{j}\right) h\left(X_{i}, X_{j}\right)-\frac{m}{2}\left\|R^{\perp}\right\|^{2} \eta\right\}=0 .
$$

ProOF. Since

$$
\left.\frac{d}{d t} \mathcal{R}_{q}^{\perp}\left[\phi_{t}\right]\right|_{t=0}=\int\left\|R^{\perp}\right\|^{q-2}\left\{\frac{q}{2} \delta\left\|R^{\perp}\right\|^{2}+\left\|R^{\perp}\right\|^{2}(\operatorname{div} T-m \tilde{g}(\eta, \zeta))\right\} d v,
$$

we need to compute $\delta\left\|R^{\perp}\right\|^{2}$. Define $D_{t}$ by

$$
D_{t}=\left(H_{t}\right)_{i k}^{\mu}\left(H_{t}\right)_{j l}^{\lambda}\left(g_{t}\right)^{k l} d x^{i} \otimes d x^{j} \otimes\left(\frac{\partial}{\partial y^{\lambda}}\right)_{\Phi} \otimes\left(\frac{\partial}{\partial y^{\mu}}\right)_{\Phi} .
$$

Then, from (1.8), we have

$$
\left\|R_{t}{ }^{\perp}\right\|^{2}=\left\{\left(D_{t}\right)_{i j}{ }^{\lambda \mu}-\left(D_{t}\right)_{j i}{ }^{\lambda \mu}\right\}\left\{\left(D_{t}\right)_{k l}{ }^{\nu \kappa}-\left(D_{t}\right)_{l k}{ }^{\nu \kappa}\right\}\left(g_{t}\right)^{i k}\left(g_{t}\right)^{j l} \tilde{g}_{\lambda \nu} \tilde{g}_{\mu \kappa} .
$$

Therefore

$$
\begin{aligned}
\frac{1}{2} \delta\left\|R^{\perp}\right\|^{2}= & \left\{(\delta D)_{i j}{ }^{\lambda \mu}-(\delta D)_{j i}{ }^{\lambda \mu}\right\}\left\{D_{k l}{ }^{\nu \kappa}-D_{l k}{ }^{\nu \kappa}\right\} g^{i k} g^{j l} \tilde{g}_{\lambda \nu} \tilde{g}_{\mu \kappa} \\
& +\left(D_{i j}{ }^{\lambda \mu}-D_{j i}{ }^{\lambda \mu}\right)\left(D_{k l}{ }^{\nu \kappa}-D_{l k}{ }^{\nu \kappa}\right)\left(\delta g^{-1}\right)^{i k} g^{j l} \tilde{g}_{\lambda \nu} \tilde{g}_{\mu \kappa} .
\end{aligned}
$$


Next we compute $\delta D$ :

$$
\begin{aligned}
\delta D= & \delta\left(H_{i k^{\mu}} H_{j l}{ }^{\lambda} g^{k l} d x^{i} \otimes d x^{j} \otimes\left(\frac{\partial}{\partial y^{\lambda}}\right)_{\phi} \otimes\left(\frac{\partial}{\partial y^{\mu}}\right)_{\phi}\right) \\
= & \left\{(\delta H)_{i k}{ }^{\mu} H_{j l}{ }^{\lambda} g^{k l}+H_{i k}{ }^{\mu}(\delta H)_{j l}{ }^{\lambda} g^{k l}+H_{i k^{\mu}} H_{j l}{ }^{\lambda}\left(\delta g^{-1}\right)^{k l}\right\} \\
& d x^{i} \otimes d x^{j} \otimes\left(\frac{\partial}{\partial y^{\lambda}}\right)_{\phi} \otimes\left(\frac{\partial}{\partial y^{\mu}}\right)_{\phi} .
\end{aligned}
$$

Using (2.5) and (2.10), we obtain

$$
\begin{aligned}
(\delta D)_{i j}{ }^{\lambda \mu} \equiv & \nabla_{i} \nabla_{k} \zeta^{v} N_{v}{ }^{\mu} H_{j}{ }^{k \lambda}+\nabla_{j} \nabla_{k} \zeta^{v} N_{v}{ }^{\lambda} H_{i}{ }^{k \mu} \\
& +c\left(H_{i j}{ }^{\lambda} N_{v}{ }^{\mu} \zeta^{v}+H_{i j}{ }^{\mu} N_{v}{ }^{\lambda} \zeta^{v}\right) \\
& +T^{l}\left(\nabla_{l} h_{i k}{ }^{v} N_{v}{ }^{\mu} h_{j}{ }^{k \lambda}+\nabla_{l} h_{j k}{ }^{v} N_{v}{ }^{\lambda} h_{i}{ }^{k \mu}\right) \\
& +\nabla_{i} T^{k} D_{j k}{ }^{\mu \lambda}+\nabla_{j} T^{k} D_{i k}{ }^{\lambda \mu} \bmod \left(d \phi^{\lambda}, d \phi^{\mu}\right) .
\end{aligned}
$$

Substituting this result into the first term of the right hand side of (2.20) and putting $D_{i j}{ }^{u v}=$ $h_{i k}{ }^{v} h_{j}{ }^{k u}$, we have

$$
\begin{aligned}
\frac{1}{2} \delta\left\|R^{\perp}\right\|^{2}= & 4 \nabla_{i} \nabla_{k} \zeta^{v} h_{j}{ }^{k u} R^{\perp i j}{ }_{u v}+2 P_{i j} h^{i j}{ }_{u} \zeta^{u} \\
& +2\left(2 T^{k} \nabla_{i} h_{k l} h_{j}^{l u}+2 \nabla_{j} T^{k} D_{i k}{ }^{u v}-\nabla_{i} T^{k} R^{\perp}{ }_{k j}{ }^{u v}\right) R^{\perp i j} u v
\end{aligned}
$$

The third term of the right hand side of (2.21) is equal to

$$
2 T^{k} R^{\perp}{ }_{i j u}{ }^{v} \nabla^{i} R^{\perp j}{ }_{k v}{ }^{u} \text {. }
$$

The second Bianchi identity for $R^{\perp}$ implies that this is equal to $\left(\nabla_{T}\left\|R^{\perp}\right\|^{2}\right) / 2$. It follows that

$$
\delta\left\|R^{\perp}\right\|^{2}=8 \nabla_{i} \nabla_{k} \zeta^{v} h_{j}{ }^{k u} R^{\perp i j}{ }_{u v}+4 P_{i j} h^{i j}{ }_{u} \zeta^{u}+T^{i} \nabla_{i}\left\|R^{\perp}\right\|^{2} .
$$

Integrating by parts and using Stokes' formula, we have

$$
\begin{aligned}
\left.\frac{d}{d t} \mathcal{R}_{q}^{\perp}\left[\phi_{t}\right]\right|_{t=0}=\int & \left\{4 q \nabla_{k} \nabla_{i}\left(\left\|R^{\perp}\right\|^{q-2} h_{j}{ }^{k u} R^{\perp i j}{ }_{u v}\right)\right. \\
& \left.+2 q\left\|R^{\perp}\right\|^{q-2} P_{i j} h^{i j}{ }_{v}-m\left\|R^{\perp}\right\|^{q} \eta_{v}\right\} \zeta^{v} d v .
\end{aligned}
$$

Here we note that we can use Stokes' formula under the assumption that $q \geq 2$. However, if $q=1$, then

$$
\nabla_{i}\left(\left\|R^{\perp}\right\|^{q-2} h_{j}{ }^{k u} R^{\perp i j}{ }_{u v}\right) \zeta^{v} \quad \text { and } \quad\left\|R^{\perp}\right\|^{q-2} h_{j}{ }^{k u} R^{\perp i j}{ }_{u v} \nabla_{k} \zeta^{v}
$$

may not converge to 0 as a point approaches to the zeros of $R^{\perp}$. Thus, when $q=1$, we need the assumption that $R^{\perp}$ does not vanish.

When $m=4$ and $q=2, \mathcal{R}_{2}^{\perp}[\phi]$ is a conformal invariant and the Yang-Mills integral in the vector bundle $T^{\perp} M$.

THEOREM 2.8. Let $\phi: M^{4} \rightarrow \tilde{M}(c)$ be an immersion of a four-dimensional compact oriented manifold $M^{4}$ into an $n$-dimensional space form $\tilde{M}(c)$. If the normal connection is self-dual or anti-self-dual, then $\phi$ is critical for the functional $\mathcal{R}_{2}^{\perp}[\phi]$. 
PROOF. Let $\varepsilon=1$ or -1 according as the normal connection is self-dual or anti-selfdual. Let $\left\{X_{1}, \ldots, X_{4}\right\}$ be an orthonormal basis in $T_{p} M$ associated with the orientation of $M^{4}$. Then the assumption that the normal connection is self-dual or anti-self-dual is equivalent to

$$
\begin{aligned}
& R^{\perp}\left(X_{1}, X_{2}\right)=\varepsilon R^{\perp}\left(X_{3}, X_{4}\right), \quad R^{\perp}\left(X_{1}, X_{3}\right)=\varepsilon R^{\perp}\left(X_{4}, X_{2}\right), \\
& R^{\perp}\left(X_{1}, X_{4}\right)=\varepsilon R^{\perp}\left(X_{2}, X_{3}\right) .
\end{aligned}
$$

(cf. [3, p. 370]) Self-dual and anti-self-dual connections are Yang-Mills instanton. Thus the normal curvature tensor $R^{\perp}$ satisfies $\nabla^{i} R^{\perp}{ }_{i j u}{ }^{v}=0$ (cf. [19, p. 21]). Thus we see from Codazzi equation (1.7) that

$$
\begin{aligned}
\square C & =-\nabla^{i} \nabla^{j}\left(h_{i}{ }^{p u} R^{\perp}{ }_{j p u}{ }^{v}\right) \\
& =-\nabla^{i}\left(h_{i}{ }^{p u} \nabla^{j} R^{\perp}{ }_{j p u}{ }^{v}\right)=0 .
\end{aligned}
$$

The second term of (2.19) vanishes. In fact,

$$
\sum_{i, j} P\left(X_{i}, X_{j}\right) h\left(X_{i}, X_{j}\right)-\left\|R^{\perp}\right\|^{2} \eta=\sum_{i, j} P\left(X_{i}, X_{j}\right) L\left(X_{i}, X_{j}\right),
$$

and (2.2) implies that $P\left(X_{i}, X_{j}\right)=\alpha \delta_{i j}$ for every $i$ and $j$, for instance

$$
\begin{aligned}
P\left(X_{1}, X_{2}\right) & =-\operatorname{trace}\left(R^{\perp}\left(X_{1}, X_{3}\right) R^{\perp}\left(X_{2}, X_{3}\right)+R^{\perp}\left(X_{1}, X_{4}\right) R^{\perp}\left(X_{2}, X_{4}\right)\right) \\
& =-\operatorname{trace}\left(R^{\perp}\left(X_{4}, X_{2}\right) R^{\perp}\left(X_{1}, X_{4}\right)+R^{\perp}\left(X_{1}, X_{4}\right) R^{\perp}\left(X_{2}, X_{4}\right)\right) \\
& =0 .
\end{aligned}
$$

3. Two-dimensional cases. Let $\phi: M^{2} \rightarrow S^{n}(c)$ be an immersion of an oriented surface $M^{2}$ into an $n$-dimensional sphere $S^{n}(c)$ of constant sectional curvature $c$. We shall rewrite (2.18), (2.18') and (2.19) in terms of the isothermal coordinate $z=x+\sqrt{-1} y$. Since $g_{11}=g_{22}=2 F$ and $g_{12}=0$, Christoffel's symbols of the Levi-Civita connection are given by

$$
\Gamma_{1}{ }_{1}{ }_{1}=-\Gamma_{2}{ }^{1}{ }_{2}=\Gamma_{1}{ }^{2}{ }_{2}=\frac{1}{2} \partial_{1} \log F, \quad \Gamma_{1}{ }^{2}{ }_{2}=-\Gamma_{1}{ }^{2}{ }_{1}=\Gamma_{2}{ }^{2}{ }_{2}=\frac{1}{2} \partial_{2} \log F,
$$

where $\partial_{1}=\partial / \partial x$ and $\partial_{2}=\partial / \partial y$. The coefficients $\Gamma_{i}^{v}$ of the normal connection with respect to an orthonormal local frame field $\left\{N_{u}\right\}$ in $T^{\perp} M$ are defined by $\nabla_{\partial_{i}}^{\perp} N_{u}=\Gamma_{i}^{v} N_{v}$ and so the relation between $\omega_{u}^{v}$ and $\Gamma_{i}^{v}$ is

$$
\Gamma_{1}^{v}=\ell_{u}^{v}+\bar{\ell}_{u}^{v}, \quad \Gamma_{2}^{v}=\sqrt{-1}\left(\ell_{u}^{v}-\bar{\ell}_{u}^{v}\right), \quad\left(\omega_{u}^{v}=\ell_{u}^{v} d z\right) .
$$

Therefore, we have

$$
\begin{aligned}
\nabla_{1}{ }^{\perp} \xi^{u} & =\partial_{1} \xi^{u}+\Gamma_{1}^{u} \xi^{v} \\
& =(\partial+\bar{\partial}) \xi^{u}+\left(\ell_{v}^{u}+\bar{\ell}_{v}^{u}\right) \xi^{v} \\
& ={ }^{\prime} \nabla^{\perp} \xi^{u}+{ }^{\prime \prime} \nabla^{\perp} \xi^{u},
\end{aligned}
$$


where we have put ${ }^{\prime} \nabla^{\perp} \xi={ }^{\prime} \nabla^{\perp} \xi^{u} d z \otimes N_{u}$ and ${ }^{\prime \prime} \nabla^{\perp} \xi={ }^{\prime \prime} \nabla^{\perp} \xi^{u} d \bar{z} \otimes N_{u}$. Similarly, we have

$$
\nabla_{2}{ }^{\perp} \xi^{u}=\sqrt{-1}\left(\nabla^{\perp} \xi^{u}-{ }^{\prime \prime} \nabla^{\perp} \xi^{u}\right) .
$$

Moreover, a straightforward computation shows that

$$
\begin{aligned}
& \nabla_{1} \nabla_{1} \xi^{u}={ }^{\prime} \nabla^{\perp \prime} \nabla^{\perp} \xi^{u}+{ }^{\prime} \nabla^{\perp \prime \prime} \nabla^{\perp} \xi^{u}+{ }^{\prime \prime} \nabla^{\perp^{\prime}} \nabla^{\perp} \xi^{u}+{ }^{\prime \prime} \nabla^{\perp^{\prime \prime}} \nabla^{\perp} \xi^{u}, \\
& \nabla_{2} \nabla_{2} \xi^{u}={ }^{\prime} \nabla^{\perp \prime} \nabla^{\perp} \xi^{u}+{ }^{\prime} \nabla^{\perp \prime} \nabla^{\perp} \xi^{u}+{ }^{\prime \prime} \nabla^{\perp \prime} \nabla^{\perp} \xi^{u}-{ }^{\prime \prime} \nabla^{\perp \prime} \nabla^{\perp} \xi^{u} .
\end{aligned}
$$

It follows that

$$
\Delta \eta=-\frac{1}{F}\left({ }^{\prime \prime} \nabla^{\perp \prime} \nabla^{\perp} \eta+^{\prime} \nabla^{\perp \prime \prime} \nabla^{\perp} \eta\right) .
$$

The tensor field $L$ has the components:

$$
L_{11}{ }^{u}=\gamma^{u}+\bar{\gamma}^{u}, \quad L_{22}{ }^{u}=-\left(\gamma^{u}+\bar{\gamma}^{u}\right), \quad L_{12}{ }^{u}=\sqrt{-1}\left(\gamma^{u}-\bar{\gamma}^{u}\right) .
$$

Therefore the components of $Q^{\perp}$ are given by

$$
Q^{\perp}{ }_{u}^{v}=\frac{1}{F^{2}}\left(\gamma^{u} \bar{\gamma}^{v}+\bar{\gamma}^{u} \gamma^{v}\right)
$$

Using (1.26), (1.28), (3.3) and (3.5) we have

$$
\begin{aligned}
-\Delta \eta+Q^{\perp} \eta & =\frac{1}{F}\left(^{\prime \prime} \nabla^{\perp \prime} \nabla^{\perp} \eta+{ }^{\prime} \nabla^{\perp \prime} \nabla^{\perp} \eta\right)+Q^{\perp} \eta \\
& =\frac{2}{F}{ }^{\prime \prime} \nabla^{\perp \prime} \nabla^{\perp} \eta+\frac{1}{F^{2}}(\langle\eta, \bar{\gamma}\rangle \gamma-\langle\eta, \gamma\rangle \bar{\gamma})+\frac{1}{F^{2}}(\langle\eta, \bar{\gamma}\rangle \gamma+\langle\eta, \gamma\rangle \bar{\gamma}) \\
& =\frac{2}{F}\left(^{\prime \prime} \nabla^{\perp \prime} \nabla^{\perp} \eta+\frac{1}{F}\langle\eta, \bar{\gamma}\rangle \gamma\right) .
\end{aligned}
$$

Thus we can rewrite (2.17) as

$$
{ }^{\prime \prime} \nabla^{\perp \prime} \nabla^{\perp} \eta+\frac{1}{F}\langle\eta, \bar{\gamma}\rangle \gamma=0
$$

or equivalently

$$
" \nabla^{\perp \prime} \nabla^{\perp} \gamma+\langle\eta, \bar{\gamma}\rangle \gamma=0
$$

which is the defining equation of Willmore surfaces $([11,25,26])$.

Next we treat (2.18), (2.18') and (2.19) with the isothermal coordinate. First, we compute the components of the tensor field $C$. Using (1.27), we have

$$
\begin{aligned}
C_{11}{ }^{v} & =R^{\perp}{ }_{1 k u}{ }^{v} h_{1 l}{ }^{u} g^{k l}=\frac{1}{2 F} R^{\perp}{ }_{12 u}{ }^{v} h_{12}{ }^{u} \\
& =\frac{1}{F} \Re^{\perp}{ }_{u}{ }^{v}\left(\gamma^{u}-\bar{\gamma}^{u}\right) .
\end{aligned}
$$


Similarly, the other components are the following:

$$
\begin{aligned}
& C_{12}{ }^{v}=\frac{\sqrt{-1}}{F} \mathfrak{R}^{\perp}{ }_{u}{ }^{v}\left(\gamma^{u}+\bar{\gamma}^{u}+2 F \eta^{u}\right), \\
& C_{21}{ }^{v}=\frac{\sqrt{-1}}{F} \mathfrak{R}^{\perp}{ }_{u}{ }^{v}\left(\gamma^{u}+\bar{\gamma}^{u}-2 F \eta^{u}\right), \\
& C_{22}{ }^{v}=-\frac{1}{F} \mathfrak{R}^{\perp}{ }_{u}{ }^{v}\left(\gamma^{u}-\bar{\gamma}^{u}\right) .
\end{aligned}
$$

Using (3.1) and (3.2), we have

$$
\begin{aligned}
& \nabla^{k} C_{j k}{ }^{v}=g^{l k}\left(\partial_{l} C_{j k}{ }^{v}-\Gamma_{l}{ }_{j} C_{i k}{ }^{v}-\Gamma_{l}{ }_{k} C_{j i}{ }^{v}+\Gamma_{l u}^{v} C_{j k}{ }^{u}\right) \\
& =\frac{1}{2 F}\left\{\partial_{1} C_{j 1}{ }^{v}+\partial_{2} C_{j 2}{ }^{v}-\left(\Gamma_{1}{ }^{i} 1+\Gamma_{2}{ }^{i}{ }_{2}\right) C_{j i}{ }^{v}-\Gamma_{1}{ }_{j}{ }_{j} C_{i 1}{ }^{v}-\Gamma_{2}{ }_{j}{ }_{j} C_{i 2}{ }^{v}\right. \\
& \left.+\Gamma_{1}^{v} C_{j 1}{ }^{u}+\Gamma_{2}^{v}{ }^{v} C_{j 2}{ }^{u}\right\} \\
& =\frac{1}{2 F}\left\{(\partial+\bar{\partial}) C_{j 1}^{v}+\sqrt{-1}(\partial-\bar{\partial}) C_{j 2}{ }^{v}\right. \\
& -\left(\Gamma_{1}{ }_{j} C_{11}{ }^{v}+\Gamma_{1}{ }^{2}{ }_{j} C_{21}{ }^{v}+\Gamma_{2}{ }^{1}{ }_{j} C_{12}{ }^{v}+\Gamma_{2}{ }^{2}{ }_{j} C_{22}{ }^{v}\right) \\
& \left.+\left(\ell_{u}^{v}+\bar{\ell}_{u}^{v}\right) C_{j 1}{ }^{u}+\sqrt{-1}\left(\ell_{u}^{v}-\bar{\ell}_{u}^{v}\right) C_{j 2}{ }^{u}\right\} \\
& =\frac{1}{2 F}\left\{\partial\left(C_{j 1}{ }^{v}+\sqrt{-1} C_{j 2}{ }^{v}\right)+\bar{\partial}\left(C_{j 1}{ }^{v}-\sqrt{-1} C_{j 2}{ }^{v}\right)+4 \sqrt{-1} \Gamma_{1}{ }_{j} \mathfrak{R}^{\perp}{ }_{u}{ }^{v} \eta^{u}\right. \\
& \left.+\ell_{u}^{v}\left(C_{j 1}^{u}+\sqrt{-1} C_{j 2}{ }^{u}\right)+\bar{\ell}_{u}^{v}\left(C_{j 1}^{u}-\sqrt{-1} C_{j 2}{ }^{u}\right)\right\} .
\end{aligned}
$$

It follows that

$$
\begin{gathered}
\nabla^{k} C_{1 k}{ }^{v}=\frac{1}{F}\left\{-\frac{1}{F}{ }^{\prime} \nabla^{\perp}\left(\mathfrak{R}^{\perp}{ }_{u}^{v} \bar{\gamma}^{u}\right)+\frac{1}{F}{ }^{\prime \prime} \nabla^{\perp}\left(\mathfrak{R}^{\perp}{ }_{u}{ }^{v} \gamma^{u}\right)\right. \\
\left.-{ }^{\prime} \nabla^{\perp}\left(\mathfrak{R}^{\perp}{ }_{u}{ }^{v} \eta^{u}\right)+{ }^{\prime \prime} \nabla^{\perp}\left(\mathfrak{R}^{\perp}{ }_{u}{ }^{v} \eta^{u}\right)\right\}, \\
\nabla^{k} C_{2 k}{ }^{v}=\frac{\sqrt{-1}}{F}\left\{\frac{1}{F}{ }^{\prime} \nabla^{\perp}\left(\mathfrak{R}^{\perp}{ }_{u}{ }^{v} \bar{\gamma}^{u}\right)+\frac{1}{F}{ }^{\prime \prime} \nabla^{\perp}\left(\mathfrak{R}^{\perp}{ }_{u}{ }^{v} \gamma^{u}\right)\right. \\
\left.-{ }^{\prime} \nabla^{\perp}\left(\mathfrak{R}^{\perp}{ }_{u}{ }^{v} \eta^{u}\right)-{ }^{\prime \prime} \nabla^{\perp}\left(\mathfrak{R}^{\perp}{ }_{u}{ }^{v} \eta^{u}\right)\right\} .
\end{gathered}
$$

Moreover, we compute $\nabla^{j} \nabla^{k} C_{j k}^{v}$. Since

$$
\begin{aligned}
\nabla^{j} \nabla^{k} C_{j k}{ }^{v} & =g^{j l} \nabla_{l} \nabla^{k} C_{j k}{ }^{v} \\
& =g^{j l}\left(\partial_{l} \nabla^{k} C_{j k}{ }^{v}-\Gamma_{l}{ }^{i}{ }_{j} \nabla^{k} C_{i k}{ }^{v}+\Gamma_{l u}^{v} \nabla^{k} C_{j k}{ }^{u}\right)
\end{aligned}
$$

and from (3.8)

$$
\nabla^{k} C_{1 k}{ }^{v}+\sqrt{-1} \nabla^{k} C_{2 k}{ }^{v}=\frac{2}{F}\left\{-\frac{1}{F}{ }^{\prime} \nabla^{\perp}\left(\mathfrak{R}^{\perp}{ }_{u}{ }^{v} \bar{\gamma}^{u}\right)+^{\prime \prime} \nabla^{\perp}\left(\mathfrak{R}^{\perp}{ }_{u}{ }^{v} \eta^{u}\right)\right\},
$$


we have

$$
\begin{aligned}
& \nabla^{j} \nabla^{k} C_{j k}{ }^{v} \\
& =\frac{1}{2 F}\left\{\partial\left(\nabla^{k} C_{1 k}{ }^{v}+\sqrt{-1} \nabla^{k} C_{2 k}{ }^{v}\right)+\bar{\partial}\left(\nabla^{k} C_{1 k}{ }^{v}-\sqrt{-1} \nabla^{k} C_{2 k}{ }^{v}\right)\right. \\
& \left.\quad+\ell_{u}^{v}\left(\nabla^{k} C_{1 k}{ }^{u}+\sqrt{-1} \nabla^{k} C_{2 k}{ }^{u}\right)+\bar{\ell}_{u}^{v}\left(\nabla^{k} C_{1 k}{ }^{u}-\sqrt{-1} \nabla^{k} C_{2 k}{ }^{u}\right)\right\}
\end{aligned}
$$

We thus obtain

$$
-\square C=\frac{2}{F^{3}} \Re\left[^{\prime \prime} \nabla^{\perp \prime} \nabla^{\perp}\left(\mathfrak{R}^{\perp} \gamma\right)\right]+\frac{1}{F^{2}}\left(\mathfrak{R}^{\perp}\right)^{2} \eta,
$$

where $\Re[$ ] means the real part of [ ].

The second term of (2.19) is computed as follows:

$$
\begin{aligned}
\sum_{i, j} P\left(X_{i}, X_{j}\right) & h\left(X_{i}, X_{j}\right)=R^{\perp}{ }_{i k u v} R^{\perp}{ }_{j}{ }^{k u v} h^{i j w} N_{w} \\
= & \frac{1}{8 F^{3}}\left(4 F^{2}\left|\Re^{\perp}\right|^{2} h_{11}{ }^{w}+4 F^{2}\left|\Re^{\perp}\right|^{2} h_{22}{ }^{w}\right) N_{w} \\
= & 2\left|\Re^{\perp}\right|^{2} \eta .
\end{aligned}
$$

Here we note that $\left|\mathfrak{R}^{\perp}\right|^{2}=F^{-2}\left\langle\mathfrak{R}^{\perp}, \overline{\mathfrak{R}}^{\perp}\right\rangle=\left\|R^{\perp}\right\|^{2} / 2$. Therefore we have obtained

THEOREM 3.1. The Euler-Lagrange equation of the functional

$$
\mathcal{R}_{2}^{\perp}[\phi]=\int\left\|R^{\perp}\right\|^{2} d v
$$

for immersion $\phi: M^{2} \rightarrow S^{n}(c)$ of an oriented surface $M^{2}$ is given by

$$
\frac{2}{F^{3}} \Re\left[^{\prime \prime} \nabla^{\perp \prime \prime} \nabla^{\perp}\left(\mathfrak{R}^{\perp} \gamma\right)\right]+\frac{1}{F^{2}}\left(\mathfrak{R}^{\perp}\right)^{2} \eta+\frac{1}{2}\left|\mathfrak{R}^{\perp}\right|^{2} \eta=0 .
$$

For the conformally invariant functional

$$
\mathcal{R}_{1}^{\perp}[\phi]=\int\left\|R^{\perp}\right\| d v
$$

the Euler-Lagrange equation is given by

$$
\square\left(\left\|R^{\perp}\right\|^{-1} C\right)=0
$$

under the condition that $R^{\perp}$ does not vanish anywhere on $M$.

COROLLARY. If the normal curvature tensor is parallel, then the immersion $\phi$ is critical for the functional $\mathcal{R}_{1}^{\perp}[\phi]$. Moreover, if $\phi$ is minimal, then $\phi$ is critical for the functional $\mathcal{R}_{2}^{\perp}[\phi]$.

Proof. We immediately have

$$
\begin{aligned}
& \nabla_{1} R^{\perp}{ }_{12 u}{ }^{v}=-2 \sqrt{-1}\left({ }^{\prime} \nabla^{\perp} \mathfrak{R}^{\perp} u^{v}+{ }^{\prime \prime} \nabla^{\perp} \mathfrak{R}^{\perp} u^{v}\right), \\
& \nabla_{2} R^{\perp} 21 u^{v}=-2\left({ }^{\prime} \nabla^{\perp} \mathfrak{R}^{\perp}{ }_{u}{ }^{v}-{ }^{\prime \prime} \nabla^{\perp} \mathfrak{R}^{\perp} u^{v}\right) .
\end{aligned}
$$


It follows that the normal curvature tensor parallel if and only if ${ }^{\prime \prime} \nabla^{\perp} \mathfrak{R}^{\perp}=0$. From the assumption, we see that ${ }^{\prime \prime} \nabla^{\perp} \mathfrak{R}^{\perp}=0$ and so $\left|\mathfrak{R}^{\perp}\right|$ is constant. If $\mathfrak{R}^{\perp}=0$, then it is trivial that $\phi$ is critical. Assume that $\mathfrak{R}^{\perp} \neq 0$. Then we see from (3.9) that (3.11) is equivalent to

$$
\frac{2}{F} \Re\left[{ }^{\prime \prime} \nabla^{\perp \prime} \nabla^{\perp}\left(\mathfrak{R}^{\perp} \gamma\right)\right]+\left(\mathfrak{R}^{\perp}\right)^{2} \eta=0 .
$$

Since

$$
\begin{aligned}
" \prime \nabla^{\perp \prime} \nabla^{\perp}\left(\mathfrak{R}^{\perp} \gamma\right) & +{ }^{\prime} \nabla^{\perp \prime} \nabla^{\perp}\left(\overline{\mathfrak{R}^{\perp}} \bar{\gamma}\right)=\mathfrak{R}^{\perp}\left(" \nabla^{\perp \prime} \nabla^{\perp} \gamma-{ }^{\prime} \nabla^{\perp \prime} \nabla^{\perp} \bar{\gamma}\right) \\
& =F \mathfrak{R}^{\perp}\left(" \nabla^{\perp \prime} \nabla^{\perp} \eta-{ }^{\prime} \nabla^{\perp \prime} \nabla^{\perp} \eta\right) \\
& =-F \mathfrak{R}^{\perp} \mathfrak{R}^{\perp} \eta,
\end{aligned}
$$

we have (3.12).

The following proposition shows that (3.12) is equivalent to the defining equation (3.7) of Willmore surfaces under appropriate assumptions.

Proposition 3.2. We assume that $\left\|R^{\perp}\right\|$ is a non-zero constant and the curvature ellipse is a circle at every point. Then (3.7) is equivalent to (3.12).

Proof. We first note that the curvature ellipse is a circle if and only if $\langle\gamma, \gamma\rangle=0$. Thus from the assumption, we have $\mathfrak{R}^{\perp} \gamma=F|\gamma|^{2} \gamma$ and

$$
\begin{aligned}
\left|\mathfrak{R}^{\perp}\right|^{2} & =F^{-2}\left\langle\mathfrak{R}^{\perp}, \overline{\mathfrak{R}}^{\perp}\right\rangle \\
& =F^{-4} \sum_{u, v}\left(\bar{\gamma}^{u} \gamma^{v}-\gamma^{u} \bar{\gamma}^{v}\right)\left(\gamma^{u} \bar{\gamma}^{v}-\bar{\gamma}^{u} \gamma^{v}\right) \\
& =2\left(|\gamma|^{4}-|\langle\gamma, \gamma\rangle|^{2}\right)=2|\gamma|^{4},
\end{aligned}
$$

which is a non-zero constant. Assume that (3.7) holds. Then

$$
\begin{aligned}
" \nabla^{\perp \prime} \nabla^{\perp}\left(\mathfrak{R}^{\perp} \gamma\right) & =F|\gamma|^{2 \prime \prime} \nabla^{\perp \prime} \nabla^{\perp} \gamma \\
& =-F|\gamma|^{2}\langle\eta, \bar{\gamma}\rangle \gamma .
\end{aligned}
$$

Therefore we have

$$
{ }^{\prime \prime} \nabla^{\perp \prime} \nabla^{\perp}\left(\mathfrak{R}^{\perp} \gamma\right)+{ }^{\prime} \nabla^{\perp \prime} \nabla^{\perp}\left(\overline{\mathfrak{R}}^{\perp} \bar{\gamma}\right)=-F|\gamma|^{2}\{\langle\eta, \bar{\gamma}\rangle \gamma+\langle\eta, \gamma\rangle \bar{\gamma}\} .
$$

Since

$$
\begin{aligned}
\left(\mathfrak{R}^{\perp}\right)^{2} \eta & =F^{-1} \mathfrak{R}^{\perp}\{\langle\eta, \bar{\gamma}\rangle \gamma-\langle\eta, \gamma\rangle \bar{\gamma}\} \\
& =\langle\eta, \bar{\gamma}\rangle|\gamma|^{2} \gamma+\langle\eta, \gamma\rangle|\gamma|^{2} \bar{\gamma},
\end{aligned}
$$

we have (3.12). Conversely, assume that (3.12) holds. Then

$$
\begin{aligned}
0 & =F^{-1}\left\{^{\prime \prime} \nabla^{\perp \prime} \nabla^{\perp}\left(\mathfrak{R}^{\perp} \gamma\right)+{ }^{\prime} \nabla^{\perp \prime} \nabla^{\perp}\left(\overline{\mathfrak{R}}^{\perp} \bar{\gamma}\right)\right\}+\left(\mathfrak{R}^{\perp}\right)^{2} \eta \\
& =|\gamma|^{2}\left({ }^{\prime \prime} \nabla^{\perp \prime \prime} \nabla^{\perp} \gamma+{ }^{\prime} \nabla^{\perp \prime} \nabla^{\perp} \bar{\gamma}\right)+|\gamma|^{2}\{\langle\eta, \bar{\gamma}\rangle \gamma+\langle\eta, \gamma\rangle \bar{\gamma}\} .
\end{aligned}
$$


Since

$$
\begin{aligned}
{ }^{\prime \prime} \nabla^{\perp \prime} \nabla^{\perp} \gamma-{ }^{\prime} \nabla^{\perp \prime} \nabla^{\perp} \bar{\gamma} & =F\left(" \nabla^{\perp \prime} \nabla^{\perp} \eta-{ }^{\prime} \nabla^{\perp \prime} \nabla^{\perp} \eta\right) \\
& =-F \Re^{\perp} \eta \\
& =\langle\eta, \gamma\rangle \bar{\gamma}-\langle\eta, \bar{\gamma}\rangle \gamma
\end{aligned}
$$

we obtain (3.7).

4. Critical surfaces. First, we shall study Willmore surfaces. Let $\phi: M \rightarrow S^{n}(c)$ be an isometric immersion of a compact oriented surface $M$ into $S^{n}(c)$. Define $\Psi \in C^{\infty}\left(E^{3,0} \otimes\right.$ $\left.\wedge^{2} \boldsymbol{C} T^{\perp} M\right)$ by $\Psi=\gamma \wedge^{\prime} \nabla^{\perp} \eta$. The immersion $\phi: M \rightarrow S^{n}(c)$ is called a $S$-Willmore surface if $\gamma \wedge \bar{\gamma} \neq 0$ and $\Psi=0$ everywhere on $M$. It is known that $S$-Willmore surfaces are Willmore surfaces and there exist Willmore surfaces which are not $S$-Willmore surfaces $([10,11])$. In the following, we shall obtain an integral formula for the sum of residues of $\operatorname{logarithmic}$ singularities of $\log |\Psi|^{2}$. We note that the Willmore surface equation (3.6) and Codazzi equation (1.25) imply that $\Psi$ is a holomorphic section of $E^{3,0} \otimes \bigwedge^{2} \boldsymbol{C} T^{\perp} M$, that is, $" \nabla^{\perp} \Psi=0$ and hence either $\Psi$ is identically zero, or else the zeros of $\Psi$ can be at most isolated. Define the symmetric product of two $p$-vectors $\xi=\xi_{1} \wedge \cdots \wedge \xi_{p}$ and $\zeta=\zeta_{1} \wedge \cdots \wedge \zeta_{p}$ in $\bigwedge C T^{\perp} M$ by

$$
\langle\xi, \zeta\rangle=\frac{1}{p !} \operatorname{det}\left(\left\langle\xi_{A}, \zeta_{B}\right\rangle\right)_{A, B=1, \ldots, p}
$$

Then we have

LEMMA 4.1. Let $\phi: M \rightarrow S^{n}(c)$ be a compact oriented Willmore surface such that $\Psi \neq 0$ identically. Let $\Sigma$ denote the set $\{p \in M \mid \Psi(p)=0\}$ and $2 j_{p}$ the real analytic order of the zero of $|\Psi|^{2}$ at $p \in \Sigma$. Set $\mathscr{N}=\sum_{p \in \Sigma} j_{p}$. Then we have

$$
-2 \pi \mathscr{N}=6 \pi \chi(M)+\int\left\{\frac{2}{|\Psi|^{2}}\left(|\Psi \gamma|^{2}-|\Psi \bar{\gamma}|^{2}\right)+\frac{A}{|\Psi|^{4}}\right\} d v,
$$

where $\Psi \gamma=\left\{\left\langle^{\prime} \nabla^{\perp} \eta, \gamma\right\rangle \gamma-\langle\gamma, \gamma\rangle^{\prime} \nabla^{\perp} \eta\right\} / 2, \Psi \bar{\gamma}=\left\{\left\langle^{\prime} \nabla^{\perp} \eta, \bar{\gamma}\right\rangle \gamma-\langle\gamma, \bar{\gamma}\rangle^{\prime} \nabla^{\perp} \eta\right\} / 2$ and $A=\left.\left.\right|^{\prime} \nabla^{\perp} \Psi\right|^{2}|\Psi|^{2}-\left|\left\langle^{\prime} \nabla^{\perp} \Psi, \bar{\Psi}\right\rangle\right|^{2}$.

Proof. On $M \backslash \Sigma$, we have

$$
\begin{aligned}
& F^{-1} \nabla^{\prime} \nabla^{\prime \prime} \log |\Psi|^{2} \\
&=\frac{F^{-4}}{|\Psi|^{4}}\left\{\left(\left\langle^{\prime} \nabla^{\perp} \Psi,{ }^{\prime \prime} \nabla^{\perp} \bar{\Psi}\right\rangle+\left\langle\Psi,{ }^{\prime} \nabla^{\perp \prime} \nabla^{\perp} \bar{\Psi}\right\rangle\right)|\Psi|^{2}-F^{-3}\left\langle\Psi,{ }^{\prime \prime} \nabla^{\perp} \bar{\Psi}\right\rangle\left\langle^{\prime} \nabla^{\perp} \Psi, \bar{\Psi}\right\rangle\right\} \\
&=\frac{1}{|\Psi|^{2}} F^{-4}\left\langle\Psi,{ }^{\prime} \nabla^{\perp \prime} \nabla^{\perp} \bar{\Psi}\right\rangle+\frac{A}{|\Psi|^{4}} .
\end{aligned}
$$


Using the Ricci identity (1.28), we have

$$
\begin{array}{rl}
3 F & K \bar{\Psi} \xi+\mathfrak{R}^{\perp} \bar{\Psi} \xi \\
& ={ }^{\prime} \nabla^{\perp \prime} \nabla^{\perp}(\bar{\Psi} \xi)-{ }^{\prime \prime} \nabla^{\perp \prime} \nabla^{\perp}(\bar{\Psi} \xi) \\
& =\left({ }^{\prime} \nabla^{\perp \prime} \nabla^{\perp} \bar{\Psi}-{ }^{\prime \prime} \nabla^{\perp \prime} \nabla^{\perp} \bar{\Psi}\right) \xi+\bar{\Psi}\left({ }^{\prime} \nabla^{\perp \prime} \nabla^{\perp} \xi-{ }^{\prime \prime} \nabla^{\perp \prime} \nabla^{\perp} \xi\right) \\
& =\left({ }^{\prime} \nabla^{\perp \prime} \nabla^{\perp} \bar{\Psi}-{ }^{\prime \prime} \nabla^{\perp \prime} \nabla^{\perp} \bar{\Psi}\right) \xi+\bar{\Psi} \mathfrak{R}^{\perp} \xi
\end{array}
$$

for every normal vector $\xi$. It follows that

$$
\nabla^{\perp \prime} \nabla^{\perp} \bar{\Psi}=3 F K \bar{\Psi}+\mathfrak{R}^{\perp} \bar{\Psi}-\bar{\Psi} \mathfrak{R}^{\perp} .
$$

Thus we obtain

$$
\begin{aligned}
F^{-4} & \left\langle\Psi,{ }^{\prime} \nabla^{\perp \prime \prime} \nabla^{\perp} \bar{\Psi}\right\rangle \\
& =3 K|\Psi|^{2}+2 F^{-4} \sum \mathfrak{R}^{\perp}{ }_{w}{ }^{u} \bar{\Psi}^{w v} \Psi^{u v} \\
& =3 K|\Psi|^{2}+2 F^{-5} \sum\left(\bar{\gamma}^{w} \gamma^{u}-\gamma^{w} \bar{\gamma}^{u}\right) \bar{\Psi}^{w v} \Psi^{u v} \\
& =3 K|\Psi|^{2}+2\left(|\Psi \gamma|^{2}-|\Psi \bar{\gamma}|^{2}\right) .
\end{aligned}
$$

The residue of the logarithmic singularities of $\log |\Psi|^{2}$ is given by

$$
-2 \pi \mathscr{N}=\lim _{\varepsilon \rightarrow 0} \int_{\Sigma_{\varepsilon}}\left(F^{-1} \nabla^{\prime} \nabla^{\prime \prime} \log |\Psi|^{2}\right) d v,
$$

where $\Sigma_{\varepsilon}$ denotes the complement in $M$ of an $\varepsilon$-neighborhood of all points of $\Sigma$. In virtue of the Gauss-Bonnet formula:

$$
\int K d v=2 \pi \chi(M)
$$

we obtain (4.2).

THEOREM 4.2. Let $\phi: M \rightarrow S^{n}(c)$ be a compact oriented Willmore surface. Assume that $\Psi \neq 0$ identically. Then we have

$$
4 \pi(3 \chi(M)+\mathscr{N}) \leq \int \frac{\left|\mathfrak{R}^{\perp}\right|^{2}}{|\gamma|^{2}} d v .
$$

The equality holds if and only if $\langle\Psi \gamma, \bar{\gamma}\rangle=0$ and ${ }^{\prime} \nabla^{\perp} \Psi$ is proportional to $\Psi$.

PROOF. We compute the first term of the integrand of (4.2) on $M \backslash \Sigma$. Since

$$
|\Psi \gamma \wedge \gamma|^{2}=\frac{1}{4}|\langle\gamma, \gamma\rangle|^{2}|\Psi|^{2},
$$

we have

$$
|\Psi \gamma|^{2}|\gamma|^{2}=\frac{1}{2}|\langle\gamma, \gamma\rangle|^{2}|\Psi|^{2}+|\langle\Psi \gamma, \bar{\gamma}\rangle|^{2} .
$$


We also have

$$
\begin{aligned}
|\Psi \bar{\gamma}|^{2} & =\frac{1}{4} F^{-7}\left\langle\left\langle^{\prime} \nabla^{\perp} \eta, \bar{\gamma}\right\rangle \gamma-\langle\gamma, \bar{\gamma}\rangle^{\prime} \nabla^{\perp} \eta,\left\langle^{\prime \prime} \nabla^{\perp} \eta, \gamma\right\rangle \bar{\gamma}-\langle\gamma, \bar{\gamma}\rangle^{\prime \prime} \nabla^{\perp} \eta\right\rangle \\
& =\frac{1}{4}\left\{\left.\left.|\gamma|^{4}\right|^{\prime} \nabla^{\perp} \eta\right|^{2}-\left|\left\langle^{\prime} \nabla^{\perp} \eta, \bar{\gamma}\right\rangle\right|^{2}|\gamma|^{2}\right\}=\frac{1}{2}|\gamma|^{2}|\Psi|^{2} .
\end{aligned}
$$

It follows that

$$
\frac{1}{|\Psi|^{2}}\left(|\Psi \gamma|^{2}-|\Psi \bar{\gamma}|^{2}\right)=-\frac{\left|\Re^{\perp}\right|^{2}}{4|\gamma|^{2}}+\frac{|\langle\Psi \gamma, \bar{\gamma}\rangle|^{2}}{|\Psi|^{2}|\gamma|^{2}}
$$

where we have used

$$
\left|\mathfrak{R}^{\perp}\right|^{2}=2\left(|\gamma|^{4}-|\langle\gamma, \gamma\rangle|^{2}\right) .
$$

Therefore we see from (4.4) and the non-negativity of $A$ that

$$
\begin{aligned}
F^{-1} \nabla^{\prime} \nabla^{\prime \prime} \log |\Psi|^{2} & =3 K-\frac{\left|\Re^{\perp}\right|^{2}}{2|\gamma|^{2}}+2 \frac{|\langle\Psi \gamma, \bar{\gamma}\rangle|^{2}}{|\Psi|^{2}|\gamma|^{2}}+\frac{A}{|\Psi|^{4}} \\
& \geq 3 K-\frac{\left|\Re^{\perp}\right|^{2}}{2|\gamma|^{2}} .
\end{aligned}
$$

Integrating (4.5), we have the desired inequality.

Surfaces with isotropic $\gamma$ in $S^{4}(c)$ are $S$-Willmore surfaces, because $\gamma$ and $\bar{\gamma}$ form an orthogonal basis of $C T^{\perp} M$ and

$$
F\left\langle^{\prime} \nabla^{\perp} \eta, \gamma\right\rangle=\left\langle^{\prime \prime} \nabla^{\perp} \gamma, \gamma\right\rangle=0 .
$$

This fact was proved in [11]. We also have

THEOREM 4.3. Let $\phi: M \rightarrow S^{5}(c)$ be a Willmore surface whose curvature ellipse is a circle everywhere. Then it is a $S$-Willmore surface.

This is immediately derived from the following lemma.

LEMMA 4.4. Let $\phi: M \rightarrow S^{6}(c)$ be a Willmore surface whose curvature ellipse is a circle everywhere. If $\Psi \neq 0$ identically, then $\gamma, \bar{\gamma},{ }^{\prime} \nabla^{\perp} \eta$ and ${ }^{\prime \prime} \nabla^{\perp} \eta$ form a basis of $C T^{\perp} M$ on $M \backslash \Sigma$ and satisfy

$$
\left\langle^{\prime} \nabla^{\perp} \eta, \gamma\right\rangle=0, \quad\left\langle^{\prime} \nabla^{\perp} \gamma, \gamma\right\rangle=0, \quad\left\langle^{\prime} \nabla^{\perp} \eta,^{\prime} \nabla^{\perp} \eta\right\rangle=0 .
$$

Proof. Since $\langle\gamma, \gamma\rangle=0$, we get

$$
\left\langle^{\prime} \nabla^{\perp} \eta, \gamma\right\rangle=0, \quad\left\langle^{\prime} \nabla^{\perp} \gamma, \gamma\right\rangle=0
$$

and so, using (3.6),

$$
\begin{aligned}
0 & =\nabla^{\prime \prime}\left\langle^{\prime} \nabla^{\perp} \eta, \gamma\right\rangle=\left\langle^{\prime \prime} \nabla^{\perp \prime} \nabla^{\perp} \eta, \gamma\right\rangle+\left\langle^{\prime} \nabla^{\perp} \eta, F^{\prime} \nabla^{\perp} \eta\right\rangle \\
& =F\left\langle^{\prime} \nabla^{\perp} \eta,{ }^{\prime} \nabla^{\perp} \eta\right\rangle .
\end{aligned}
$$


Therefore we see that the subspace spanned by $\gamma$ and ${ }^{\prime} \nabla^{\perp} \eta$ in $C T^{\perp} M$ is isotropic. Since $\Psi=\gamma \wedge^{\prime} \nabla^{\perp} \eta \neq 0$, vectors $\gamma, \bar{\gamma},{ }^{\prime} \nabla^{\perp} \eta$ and ${ }^{\prime \prime} \nabla^{\perp} \eta$ form a basis of $C T^{\perp} M$ on $M \backslash \Sigma$.

THEOREM 4.5. Let $\phi: M \rightarrow S^{6}(1)$ be a compact oriented Willmore surface. Assume that $\Psi \neq 0$ identically and the curvature ellipse is a circle everywhere. Then we have

$$
-2 \pi \mathscr{N}=6 \pi \chi(M)-\int|\gamma|^{2} d v+\int \frac{|\alpha|^{2}}{|\Psi|^{2}} d v,
$$

where $\alpha=\left\langle\nabla^{\prime} \nabla^{\prime \prime} \nabla^{\perp} \eta, \gamma\right\rangle=-\left\langle^{\prime} \nabla^{\perp} \eta,{ }^{\prime} \nabla^{\perp} \gamma\right\rangle$. In particular, if $M$ is a topological sphere, then we have

$$
\int|\gamma|^{2} d v=12 \pi+2 \pi \mathscr{N}
$$

Proof. By Lemma 4.4, we can set

$$
\begin{aligned}
{ }^{\prime} \nabla^{\perp \prime} \nabla^{\perp} \eta & =a \gamma+b \bar{\gamma}+c^{\prime} \nabla^{\perp} \eta+d^{\prime \prime} \nabla^{\perp} \eta, \\
{ }^{\prime} \nabla^{\perp} \gamma & =a^{\prime} \gamma+b^{\prime} \bar{\gamma}+c^{\prime \prime} \nabla^{\perp} \eta+d^{\prime \prime \prime} \nabla^{\perp} \eta .
\end{aligned}
$$

Taking the symmetric product of the both hand sides of the above equation and $\gamma\left({ }^{\prime} \nabla^{\perp} \eta\right)$, we obtain

$$
\begin{array}{rlrl}
F^{2} b & =\frac{\left.\left.\alpha\right|^{\prime} \nabla^{\perp} \eta\right|^{2}}{2|\Psi|^{2}}, & F^{3} d=\frac{-\alpha\left\langle^{\prime} \nabla^{\perp} \eta, \bar{\gamma}\right\rangle}{2|\Psi|^{2}}, \\
F^{3} b^{\prime}=\frac{\alpha\left\langle^{\prime \prime} \nabla^{\perp} \eta, \gamma\right\rangle}{2|\Psi|^{2}}, & F d^{\prime}=\frac{-\alpha|\gamma|^{2}}{2|\Psi|^{2}} .
\end{array}
$$

It follows that

$$
\begin{aligned}
{ }^{\prime} \nabla^{\perp \prime} \nabla^{\perp} \eta \wedge \Psi & =\frac{\alpha F^{-3}}{2|\Psi|^{2}}\left\{\left.\left.F\right|^{\prime} \nabla^{\perp} \eta\right|^{2} \bar{\gamma} \wedge \Psi-\left\langle{ }^{\prime} \nabla^{\perp} \eta, \bar{\gamma}\right\rangle^{\prime \prime} \nabla^{\perp} \eta \wedge \Psi\right\}, \\
{ }^{\prime} \nabla^{\perp} \gamma \wedge \Psi & =\frac{\alpha F^{-3}}{2|\Psi|^{2}}\left\{\left\langle^{\prime \prime} \nabla^{\perp} \eta, \gamma\right\rangle \bar{\gamma} \wedge \Psi-F^{2}|\gamma|^{2}{ }^{\prime \prime} \nabla^{\perp} \eta \wedge \Psi\right\} .
\end{aligned}
$$

The following general formula for decomposable 2 -vectors are easily proved:

$$
\begin{aligned}
& \left\langle p \wedge s_{1}, \bar{q} \wedge \bar{t}_{1}\right\rangle\left\langle p \wedge s_{2}, \bar{q} \wedge \bar{t}_{2}\right\rangle-\left\langle p \wedge s_{1}, \bar{q} \wedge \bar{t}_{2}\right\rangle\left\langle p \wedge s_{2}, \bar{q} \wedge \bar{t}_{1}\right\rangle \\
& =\frac{3}{2}\langle p, \bar{q}\rangle\left\langle p \wedge s_{1} \wedge s_{2}, \bar{q} \wedge \bar{t}_{1} \wedge \bar{t}_{2}\right\rangle .
\end{aligned}
$$

Using this formula, we compute $A$ defined in Lemma 4.1. We have

$$
{ }^{\prime} \nabla^{\perp} \Psi=\theta \wedge \delta+\gamma \wedge \omega,
$$


where we have put $\theta={ }^{\prime} \nabla^{\perp} \gamma, \delta={ }^{\prime} \nabla^{\perp} \eta$ and $\omega={ }^{\prime} \nabla^{\perp \prime} \nabla^{\perp} \eta$. Then $A$ is computed as follows:

$$
\begin{aligned}
A= & |\theta \wedge \delta+\gamma \wedge \omega|^{2}|\gamma \wedge \delta|^{2}-|\langle\theta \wedge \delta+\gamma \wedge \omega, \bar{\gamma} \wedge \bar{\delta}\rangle|^{2} \\
= & |\theta \wedge \delta|^{2}|\gamma \wedge \delta|^{2}-|\langle\theta \wedge \delta, \bar{\gamma} \wedge \bar{\delta}\rangle|^{2} \\
& +|\gamma \wedge \omega|^{2}|\gamma \wedge \delta|^{2}-|\langle\gamma \wedge \omega, \bar{\gamma} \wedge \bar{\delta}\rangle|^{2} \\
& +F^{-4}\langle\theta \wedge \delta, \bar{\gamma} \wedge \bar{\omega}\rangle|\gamma \wedge \delta|^{2}-F^{-7}\langle\theta \wedge \delta, \bar{\gamma} \wedge \bar{\delta}\rangle\langle\bar{\gamma} \wedge \bar{\omega}, \gamma \wedge \delta\rangle \\
& +F^{-4}\langle\gamma \wedge \omega, \bar{\theta} \wedge \bar{\delta}\rangle|\gamma \wedge \delta|^{2}-F^{-7}\langle\gamma \wedge \omega, \bar{\gamma} \wedge \bar{\delta}\rangle\langle\bar{\theta} \wedge \bar{\delta}, \gamma \wedge \delta\rangle \\
= & \frac{3}{2}\left\{|\delta|^{2}|\delta \wedge \theta \wedge \gamma|^{2}+|\gamma|^{2}|\gamma \wedge \omega \wedge \delta|^{2}\right. \\
& \left.\quad-2 \Re\left[F^{-7}\langle\bar{\delta}, \gamma\rangle\langle\omega \wedge \gamma \wedge \delta, \bar{\theta} \wedge \bar{\gamma} \wedge \bar{\delta}\rangle\right]\right\} .
\end{aligned}
$$

Thus we have

$$
\begin{aligned}
A=\frac{3}{2}\left\{\left.\left.|\gamma|^{2}\right|^{\prime} \nabla^{\perp \prime} \nabla^{\perp} \eta \wedge \Psi\right|^{2}+\left.\left.\left.\left.\right|^{\prime} \nabla^{\perp} \eta\right|^{2}\right|^{\prime} \nabla^{\perp} \gamma \wedge \Psi\right|^{2}\right. \\
\left.\quad-2 \Re\left[F^{-7}\left\langle\gamma,{ }^{\prime \prime} \nabla^{\perp} \eta\right\rangle\left\langle^{\prime} \nabla^{\perp \prime} \nabla^{\perp} \eta \wedge \Psi,{ }^{\prime \prime} \nabla^{\perp} \bar{\gamma} \wedge \bar{\Psi}\right\rangle\right]\right\} .
\end{aligned}
$$

To compute three terms of the right hand side of (4.11), we use (4.1) and (4.9). The first term is computed as follows:

$$
\begin{aligned}
& \left.\left.|\gamma|^{2}\right|^{\prime} \nabla^{\perp \prime} \nabla^{\perp} \eta \wedge \Psi\right|^{2} \\
& =\frac{|\gamma|^{2}|\alpha|^{2}}{4|\Psi|^{4}}\left\langle\left.\left.\right|^{\prime} \nabla^{\perp} \eta\right|^{2} \bar{\gamma} \wedge^{\prime} \nabla^{\perp} \eta \wedge \gamma-\left\langle^{\prime} \nabla^{\perp} \eta, \bar{\gamma}\right\rangle^{\prime \prime} \nabla^{\perp} \eta \wedge^{\prime} \nabla^{\perp} \eta \wedge \gamma,\right. \\
& \left.\left.\left.\quad\right|^{\prime} \nabla^{\perp} \eta\right|^{2} \gamma \wedge^{\prime \prime} \nabla^{\perp} \eta \wedge \bar{\gamma}-\left\langle^{\prime \prime} \nabla^{\perp} \eta, \gamma\right\rangle^{\prime} \nabla^{\perp} \eta \wedge^{\prime \prime} \nabla^{\perp} \eta \wedge \bar{\gamma}\right\rangle \\
& =\frac{|\gamma|^{2}|\alpha|^{2}}{12|\Psi|^{4}}\left\{\left.\left.\right|^{\prime} \nabla^{\perp} \eta\right|^{4}|\gamma|^{2}|\Psi|^{2}-\left.\left.\right|^{\prime} \nabla^{\perp} \eta\right|^{2}\left|\left\langle^{\prime} \nabla^{\perp} \eta, \bar{\gamma}\right\rangle\right|^{2}|\Psi|^{2}\right\} \\
& =\left.\left.\frac{|\gamma|^{2}|\alpha|^{2}}{12|\Psi|^{2}}\right|^{\prime} \nabla^{\perp} \eta\right|^{2}\left\{\left.\left.\right|^{\prime} \nabla^{\perp} \eta\right|^{2}|\gamma|^{2}-\left|\left\langle^{\prime} \nabla^{\perp} \eta, \bar{\gamma}\right\rangle\right|^{2}\right\} \\
& =\left.\left.\frac{1}{6}|\gamma|^{2}|\alpha|^{2}\right|^{\prime} \nabla^{\perp} \eta\right|^{2} .
\end{aligned}
$$

Similarly, the second and third terms become

$$
\begin{gathered}
\left.\left.\left.\left.\right|^{\prime} \nabla^{\perp} \eta\right|^{2}\right|^{\prime} \nabla^{\perp} \gamma \wedge \Psi\right|^{2}=\left.\left.\frac{1}{6}\right|^{\prime} \nabla^{\perp} \eta\right|^{2}|\gamma|^{2}|\alpha|^{2}, \\
2 \Re\left[F^{-7}\left\langle\gamma,{ }^{\prime \prime} \nabla^{\perp} \eta\right\rangle\left\langle^{\prime} \nabla^{\perp \prime} \nabla^{\perp} \eta \wedge \Psi,{ }^{\prime \prime} \nabla^{\perp} \bar{\gamma} \wedge \bar{\Psi}\right\rangle\right]=\frac{1}{3}|\alpha|^{2}\left|\left\langle^{\prime} \nabla^{\perp} \eta, \bar{\gamma}\right\rangle\right|^{2} .
\end{gathered}
$$

Substituting these equations into (4.11), we get $A=|\alpha|^{2}|\Psi|^{2}$. Since $\Psi \gamma=0$, (4.2) reduces to (4.7). 
Furthermore, if $M$ is a topological sphere, then $\alpha$ vanishes. To prove this result, we have only to show that $\alpha$ is a holomorphic differential of degree 4. By (3.6), we obtain

$$
\begin{aligned}
\nabla^{\prime \prime} & \left\langle^{\prime} \nabla^{\perp} \eta,{ }^{\prime} \nabla^{\perp} \gamma\right\rangle \\
& =\left\langle^{\prime \prime} \nabla^{\perp \prime} \nabla^{\perp} \eta,{ }^{\prime} \nabla^{\perp} \gamma\right\rangle+\left\langle^{\prime} \nabla^{\perp} \eta,{ }^{\prime \prime} \nabla^{\perp \prime} \nabla^{\perp} \gamma\right\rangle \\
& =-F^{-1}\langle\bar{\gamma}, \eta\rangle\left\langle\gamma,{ }^{\prime} \nabla^{\perp} \gamma\right\rangle+\left\langle^{\prime} \nabla^{\perp} \eta, F^{\prime} \nabla^{\perp \prime} \nabla^{\perp} \eta+2 K F \gamma-\mathfrak{R}^{\perp} \gamma\right\rangle \\
& =\frac{1}{2} F \nabla^{\prime}\left\langle^{\prime} \nabla^{\perp} \eta,{ }^{\prime} \nabla^{\perp} \eta\right\rangle=0 .
\end{aligned}
$$

Second, we study surfaces satisfying (3.10). If the normal connection is flat, then (3.10) trivially holds. By the same proof as that of Proposition 3.2, we obtain

LEMMA 4.6. Under the assumption that the curvature ellipses are circles of constant radius on $M$, (3.10) is equivalent to

$$
{ }^{\prime \prime} \nabla^{\perp \prime} \nabla^{\perp} \eta+F^{-1}\langle\eta, \bar{\gamma}\rangle \gamma+\frac{F}{2}|\gamma|^{2} \eta=0 .
$$

ProOF. Since $\mathfrak{R}^{\perp} \gamma=F|\gamma|^{2} \gamma$ and $|\gamma|$ is constant, we have

$$
" \nabla^{\perp \prime} \nabla^{\perp}\left(\mathfrak{R}^{\perp} \gamma\right)=F|\gamma|^{2 "} \nabla^{\perp \prime} \nabla^{\perp} \gamma .
$$

Using

$$
\left(\Re^{\perp}\right)^{2} \eta=|\gamma|^{2}(\langle\bar{\gamma}, \eta\rangle \gamma+\langle\gamma, \eta\rangle \bar{\gamma}),
$$

we obtain

$$
\begin{aligned}
\frac{1}{F}\left\{\left\{^{\prime \prime} \nabla^{\perp \prime} \nabla^{\perp}\left(\mathfrak{R}^{\perp} \gamma\right)+{ }^{\prime} \nabla^{\perp \prime} \nabla^{\perp}\left(\overline{\mathfrak{R}^{\perp}} \bar{\gamma}\right)\right\}+\left(\mathfrak{R}^{\perp}\right)^{2} \eta\right. \\
=|\gamma|^{2}\left({ }^{\prime \prime} \nabla^{\perp \prime \prime} \nabla^{\perp} \gamma+{ }^{\prime} \nabla^{\perp \prime} \nabla^{\perp} \bar{\gamma}+\langle\bar{\gamma}, \eta\rangle \gamma+\langle\gamma, \eta\rangle \bar{\gamma}\right) \\
\left.=|\gamma|^{2}\left\{F{ }^{\prime \prime} \nabla^{\perp \prime} \nabla^{\perp} \eta+{ }^{\prime} \nabla^{\perp \prime} \nabla^{\perp} \eta\right)+\langle\bar{\gamma}, \eta\rangle \gamma+\langle\gamma, \eta\rangle \bar{\gamma}\right\} .
\end{aligned}
$$

It follows from the Ricci identity (1.28) that

$$
\begin{aligned}
\left.\frac{2}{F} \Re L^{\prime \prime} \nabla^{\perp \prime} \nabla^{\perp}\left(\mathfrak{R}^{\perp} \gamma\right)\right]+\left(\mathfrak{R}^{\perp}\right)^{2} \eta+\frac{F^{2}}{2}\left|\mathfrak{R}^{\perp}\right|^{2} \eta \\
=|\gamma|^{2}\left\{F\left(2^{\prime \prime} \nabla^{\perp \prime} \nabla^{\perp} \eta+\mathfrak{R}^{\perp} \eta\right)+\langle\bar{\gamma}, \eta\rangle \gamma+\langle\gamma, \eta\rangle \bar{\gamma}+F^{2}|\gamma|^{2} \eta\right\} \\
=2 F|\gamma|^{2}\left(" \nabla^{\perp \prime} \nabla^{\perp} \eta+F^{-1}\langle\bar{\gamma}, \eta\rangle \gamma+\frac{F}{2}|\gamma|^{2} \eta\right) .
\end{aligned}
$$

When $\gamma=0, \phi$ is totally umbilical and hence (4.12) holds. Thus (3.10) is equivalent to (4.12).

An isometric immersion $\phi: M \rightarrow \tilde{M}$ is said to be constant isotropic if $\|H(X, X)\|^{2}$ is constant on the unit tangent bundle of $M$. In the case that $M$ is a surface, we easily see that $\phi$ is constant isotropic if and only if it is pseudo-umbilical $(\langle\gamma, \eta\rangle=0)$, the curvature ellipses are circles $(\langle\gamma, \gamma\rangle=0)$ and $\|\eta\|^{2}+|\gamma|^{2} / 2$ is constant. In [23], we determined constant isotropic 
surfaces in $S^{5}(c)$. All of them are of constant Gauss curvature. In connection with this result, we state

THEOREM 4.7. Let $\phi: M \rightarrow S^{n}(c)$ be a pseudo-umbilical immersion of a surface $M$. If the curvature ellipses are circles of constant radius on $M$ and $\phi$ satisfies (3.10), then $M$ is of constant Gauss curvature.

ProOF. Since $\left\langle^{\prime} \nabla^{\perp} \eta, \gamma\right\rangle=0$, we have, from (4.12),

$$
\begin{aligned}
0 & =\nabla^{\prime \prime}\left\langle^{\prime} \nabla^{\perp} \eta, \gamma\right\rangle \\
& =\left\langle^{\prime \prime} \nabla^{\perp \prime} \nabla^{\perp} \eta, \gamma\right\rangle+\left\langle^{\prime} \nabla^{\perp} \eta, F^{\prime} \nabla^{\perp} \eta\right\rangle \\
& =-\frac{F}{2}|\gamma|^{2}\langle\eta, \gamma\rangle+F\left\langle^{\prime} \nabla^{\perp} \eta,{ }^{\prime} \nabla^{\perp} \eta\right\rangle \\
& =F\left\langle^{\prime} \nabla^{\perp} \eta,{ }^{\perp} \eta\right\rangle .
\end{aligned}
$$

Thus we see that

$$
\begin{aligned}
0 & =\nabla^{\prime \prime}\left\langle^{\prime} \nabla^{\perp} \eta,{ }^{\prime} \nabla^{\perp} \eta\right\rangle \\
& =-|\gamma|^{2}\left\langle\eta,{ }^{\prime} \nabla^{\perp} \eta\right\rangle=-\frac{1}{2}|\gamma|^{2} \nabla^{\prime}\|\eta\|^{2} .
\end{aligned}
$$

If $\gamma=0$, then $\phi$ is totally umbilical. If $\|\eta\|^{2}$ is constant, then $K$ is constant because of the Gauss equation (1.24).

REMARK. If $\phi: M \rightarrow S^{n}(c)$ is a constant isotropic minimal immersion, then the assumption that $\phi$ is pseudo-umbilical and satisfies (3.10) is trivially satisfied (cf. (4.12)). Minimal surfaces of constant Gauss curvature in $S^{n}(c)$ were determined in [6].

In the next theorem, we characterize a part of minimal surfaces of constant Gauss curvature in $S^{n}(c)$ by the conditions that $M$ is compact, $\phi$ is critical for the functional $\mathcal{R}_{2}^{\perp}$ and the curvature ellipses are circles everywhere.

THEOREM 4.8. Let $\phi: M \rightarrow S^{n}(c)$ be a minimal immersion of compact surface $M$. If $\phi$ satisfies (3.10) and the curvature ellipses are circles everywhere, then the Gauss curvature of $M$ is constant and the immersion is a standard minimal immersion of a sphere or a constant isotropic minimal immersion of a flat torus (cf. [6, 17, 23]).

Proof. If $\mathfrak{R}^{\perp} \equiv 0$, then $\gamma=0$ and so $\phi$ is totally umbilical. Assume that $\mathfrak{R}^{\perp}$ does not vanish identically. Equation (3.10) reduces to

$$
\mathfrak{R}\left[^{\prime \prime} \nabla^{\perp \prime \prime} \nabla^{\perp}\left(\mathfrak{R}^{\perp} \gamma\right)\right]=0 .
$$

It follows that

$$
\left(\nabla^{\prime \prime} \nabla^{\prime \prime}|\gamma|^{2}\right) \gamma+\left(\nabla^{\prime} \nabla^{\prime}|\gamma|^{2}\right) \bar{\gamma}=0 .
$$

Vectors $\gamma$ and $\bar{\gamma}$ are linearly independent on $\Sigma^{\prime}=\left\{p \in M \mid \mathfrak{R}^{\perp}(p) \neq 0\right\}$. Thus $\nabla^{\prime} \nabla^{\prime}|\gamma|^{2}=0$ on $\Sigma^{\prime}$. Since $\phi$ is real analytic, $M \backslash \Sigma^{\prime}$ is discrete in $M$. Hence $\nabla^{\prime} \nabla^{\prime}|\gamma|^{2}=0$ on $M$. It follows that $\nabla^{\prime} \nabla^{\prime} K=0$ on $M$ in virtue of the Gauss equation (1.24). In the subsequent 
sections, we study a two-dimensional Riemannian manifold $(M, g)$ which admits a function satisfying $\nabla \nabla f=\tau g$. In Section 6, we prove that if the Gauss curvature $K$ of a compact two-dimensional Riemannian manifold satisfies $\nabla^{\prime} \nabla^{\prime} K=0$, then $K$ is constant. From this and the result of [6], we have the assertion.

REMARK. For any standard minimal immersion of a sphere, the curvature ellipses are circles. On the other hand, there are minimal immersions of flat tori such that curvature ellipses are not circles (cf. [6]).

Next, let us assume that the mean curvature vector of the immersion $\phi: M \rightarrow S^{n}(c)$ is parallel. Chen [9] and Yau [27] proved that if the mean curvature vector of $\phi$ is parallel, then $M$ is one of the following surfaces:

(1) a minimal surface in $S^{n}(c)$,

(2) a minimal surface of a small hypersphere of $S^{n}(c)$,

(3) a surface with constant mean curvature in a three-dimensional sphere of $S^{n}(c)$.

In the following collorary, we show that if $\phi$ is not totally umbilical, then the conditon for the curvature ellipses excludes the case (3) and the equation (3.10) excludes the case (2) from our conclusion.

COROLLARY. Let $\phi: M \rightarrow S^{n}(c)$ be an immersion of a compact surface $M$. If $\phi$ satisfies (3.10), the mean curvature vector is parallel and the curvature ellipses are circles everywhere, then the Gauss curvature of $M$ is constant and the immersion is a standard minimal immersion of a sphere, a constant isotropic minimal immersion of a flat torus or a totally umbilical immersion.

Proof. In the case (3), the normal connection is flat (cf. [9, p. 106]). Since $\langle\gamma, \gamma\rangle=0$, we have $\gamma=0$. Thus $\phi$ is totally umbilical in the case (3). If $\mathfrak{R}^{\perp}=0$ in the case (2), then $\gamma=0$ and hence $\phi$ is totally umbilical. Assume that $\mathfrak{R}^{\perp}$ does not vanish identically in the case (2). Take the symmetric product of both hand sides of (3.10) and $\eta$. Then we have

$$
2 \mathfrak{R}\left[F^{-3} \nabla^{\prime \prime} \nabla^{\prime \prime}\left\langle\mathfrak{R}^{\perp} \gamma, \eta\right\rangle\right]+F^{-2}\left\langle\left(\mathfrak{R}^{\perp}\right)^{2} \eta, \eta\right\rangle+\frac{1}{2}\left|\mathfrak{R}^{\perp}\right|^{2}\|\eta\|^{2}=0 .
$$

Since

$$
\mathfrak{R}^{\perp} \eta={ }^{\prime} \nabla^{\perp \prime} \nabla^{\perp} \eta-{ }^{\prime \prime} \nabla^{\perp \prime} \nabla^{\perp} \eta=0,
$$

we see that $\eta=0$ on the open dense set $\Sigma^{\prime}$, where we note that $\phi$ is real analytic. Therefore we have $\eta=0$ on $M$.

5. Equation $\nabla \nabla f=\tau g$. In the proof of Theorem 4.8, we used the result that a compact surface whose Gauss curvature satisfies $\nabla^{\prime} \nabla^{\prime} K=0$ is of constant curvature. The equation $\nabla^{\prime} \nabla^{\prime} K=0$ can be rewritten as a tensor equation $\nabla \nabla K=\tau g$, $\tau$ being a $C^{\infty}$ function on $M$. In the present and next sections, we shall study a complete two-dimensional Riemannian manifold $M$ which admits a function $f$ satisfying $\nabla \nabla f=\tau g$ (cf. $[4,15,18$, 24]). 
Let $M$ be a two-dimensional $C^{\infty}$ manifold. We assume that $M$ is compact and orientable. Let $\mathcal{M}$ denote the set of Riemannian metrics on $M$. Furthermore, let $\mathcal{M}_{1}$ denote the subset $\left\{g \in \mathcal{M} \mid \int_{M} d v_{g}=1\right\}$ and $\mathcal{M}_{2}$ the subset $\left\{g \in \mathcal{M}_{1} \mid d v_{g}=\mu\right\}$, where $\mu$ is a positive density on $M$ with total volume $\int_{M} \mu=1$ (cf. [3]). In the compact open $C^{\infty}$ topology, $\mathcal{M}$ is an open convex cone in the set $C^{\infty}\left(S^{2} T^{*} M\right)$ of all $C^{\infty}$ sections of $S^{2} T^{*} M$. We consider the following functional $\mathcal{F}_{J}$ from $\mathcal{M}$ to $\boldsymbol{R}$ :

$$
\mathcal{F}_{J}[g]=\int_{M} J(K) d v_{g},
$$

where $J=J(x)$ is a function defined on $\boldsymbol{R}, J(K)$ the composition $J \circ K$ and $d v_{g}$ the area element of $g \in \mathcal{M}$. The Euler-Lagrange equation is given by

$$
\nabla \nabla \dot{J}(K)+\{\triangle \dot{J}(K)-K \dot{J}(K)+J(K)\} g=0
$$

for a critical point $g \in \mathcal{M}$, where $\nabla$ denotes the covariant derivative with respect to $g$, the Laplace operator is defined by $\Delta=-g^{i j} \nabla_{i} \nabla_{j}$ and $\dot{J}(K)$ the composite $\dot{J} \circ K$. The equation (5.1) for the case $J(x)=x^{2}$ is well-known (cf. [3], Chapter 4). However, for the sake of completeness, we give the proof in the following.

Let $g(t)$ be a smooth curve $(-\varepsilon, \varepsilon) \rightarrow \mathcal{M}$ such that $g(0)=g$. We compute $\mathcal{F}_{J}^{\prime}[g]:=$ $\left(d / d t \mathcal{F}_{J}[g(t)]\right)(0)$. Since

$$
\mathcal{F}_{J}^{\prime}[g]=\int_{M} \dot{J}(K) \frac{\partial K}{\partial t}(0) d v_{g}+\int_{M} J(K)\left(d v_{g(t)}\right)^{\prime}(0),
$$

we have to compute $(\partial K / \partial t)(0)$ and $\left(d v_{g(t)}\right)^{\prime}(0)$. Let $k \in T_{g} \mathcal{M}$ be defined by $k=g^{\prime}(0)$. Then it is easy to show that

$$
\left(\frac{\partial}{\partial t} g^{i j}\right)(0)=-k^{i j}
$$

and hence

$$
\mathfrak{g}^{\prime}(0)=(\operatorname{tr} k) \mathfrak{g},
$$

where $\mathfrak{g}(t)=\operatorname{det}\left(g_{i j}(t)\right), \mathfrak{g}=\mathfrak{g}(0)$ and trk $=k_{i j} g^{i j}$. Therefore we have

$$
\left(d v_{g(t)}\right)^{\prime}(0)=\frac{\mathfrak{g}^{\prime}(0)}{2 \sqrt{\mathfrak{g}}} d x^{1} \wedge d x^{2}=\frac{1}{2} \operatorname{tr} k d v_{g} .
$$

The derivative $\left(\partial \Gamma_{j}{ }_{k}{ }_{k} / \partial t\right)(0)$ of the coefficients of Riemannian connection $\nabla$ is given by

$$
\left(\frac{\partial}{\partial t} \Gamma_{j k}{ }^{i}\right)(0)=\frac{1}{2} g^{i p}\left(\nabla_{j} k_{p k}+\nabla_{k} k_{j p}-\nabla_{p} k_{j k}\right) .
$$

Using this equation in the derivation of the Rimannian curvature tensor $R_{i j k}{ }^{l}$ :

$$
\begin{aligned}
\frac{\partial}{\partial t}\left\{K\left(g_{j k} \delta_{i}^{l}-g_{i k} \delta_{j}^{l}\right)\right\} & =\left(\frac{\partial}{\partial t} R_{i j k}{ }^{l}\right)(0) \\
& =\left\{\frac{\partial}{\partial t}\left(\frac{\partial}{\partial x^{i}} \Gamma_{j}^{l}{ }_{k}-\frac{\partial}{\partial x^{j}} \Gamma_{i}^{l}{ }_{k}+\Gamma_{i}^{l}{ }_{p} \Gamma_{j}{ }^{p}{ }_{k}-\Gamma_{j}{ }^{l}{ }_{p} \Gamma_{i}{ }^{p} k\right)\right\}(0),
\end{aligned}
$$


we get

$$
2\left(\frac{\partial}{\partial t} K\right)(0)=\nabla_{i} \nabla_{j} k^{i j}+\Delta \operatorname{tr} k-K \operatorname{tr} k .
$$

By integration by parts, we have

$$
\begin{aligned}
2 \mathcal{F}_{J}^{\prime}[g] & =\int_{M}\left\{\dot{J}(K)\left(\nabla_{i} \nabla_{j} k^{i j}+\Delta \operatorname{tr} k-K \operatorname{tr} k\right)+J(K) \operatorname{tr} k\right\} d v_{g} \\
& =\int_{M}\left[\nabla_{i} \nabla_{j} \dot{J}(K)+\{\Delta \dot{J}(K)-K \dot{J}(K)+J(K)\} g_{i j}\right] k^{i j} d v_{g} .
\end{aligned}
$$

The equation (5.1) is the necessary and sufficient condition for that $\mathcal{F}_{J}^{\prime}[g]=0$ for arbitrary $k \in T_{g} \mathcal{M}$. The equation (5.1) implies that

$$
\Delta \dot{J}(K)=2\{K \dot{J}(K)-J(K)\}
$$

and hence is rewritten as

$$
\nabla \nabla \dot{J}(K)=\{J(K)-K \dot{J}(K)\} g .
$$

We now introduce $C^{\infty}$ functions on $M$

$$
f=\dot{J}(K), \quad \tau=J(K)-K \dot{J}(K) .
$$

Then (5.3) becomes

$$
\nabla \nabla f=\tau g
$$

which shows that $f$ is a concircular scalar field on $M$ (cf. [24]).

Recall that $T_{g} \mathcal{M}_{1}=\left\{k \in T_{g} \mathcal{M} \mid \int \operatorname{tr} k d v_{g}=0\right\}$ and $T_{g} \mathcal{M}_{2}=\left\{k \in T_{g} \mathcal{M} \mid \operatorname{tr} k=0\right\}$. Thus $g$ is a critical point of $\left.\mathcal{F}_{J}\right|_{\mathcal{M}_{1}}$ (resp. $\left.\mathcal{F}_{J}\right|_{\mathcal{M}_{2}}$ ) if and only if the orthogonal projection of the left hand side of (5.1) onto $T_{g} \mathcal{M}_{1}$ (resp. $T_{g} \mathcal{M}_{2}$ ) is zero. Thus if $g$ is a critical point for the functionals $\mathcal{F}_{J},\left.\mathcal{F}_{J}\right|_{\mathcal{M}_{1}}$ or $\left.\mathcal{F}_{J}\right|_{\mathcal{M}_{2}}$, then we have a concircular scalar field on $M$. The function $\tau$, called the characteristic function of $f$, can be considered as a function $\tau(f)$ of $f$ if $\dot{J}$ is strictly monotone, i.e., $\ddot{J} \neq 0$ anywhere on $\boldsymbol{R}$. In fact, we have $\tau=-\Delta f / 2$ and covariantly differentiating the both hand sides of (5.4) and using the Ricci identity, we obtain

$$
\nabla \Delta f=2 K \nabla f .
$$

Under the assumption that $\ddot{J} \neq 0, K$ is represented as $K=U(f)$ with $U=\dot{J}^{-1}$. Thus $\tau$ is given by

$$
\tau=-\int U(f) d f,
$$

where the integral constant is chosen in such away that $\int_{M} \tau d v_{g}=0$. Since

$$
\nabla\|\nabla f\|^{2}=2 \tau(f) \nabla f,
$$

the length of $\nabla f$ is given by

$$
\|\nabla f\|^{2}=2 \int \tau(f) d f
$$


We have shown the following.

THEOREM 5.1. The critical point $g \in \mathcal{M}$ of the functional $\mathcal{F}_{J}$ is characterized by (5.3). If $g$ is a critical point for $\mathcal{F}_{J},\left.\mathcal{F}_{J}\right|_{\mathcal{M}_{1}}$, or $\left.\mathcal{F}_{J}\right|_{\mathcal{M}_{2}}$, then the function $f:=\dot{J}(K)$ is a concircular scalar field on $M$. If $\ddot{J} \neq 0$, then the characteristic function $\tau$ and the length of $\nabla f$ are given by (5.5) and (5.6), respectively.

Next, let $M$ be a complete two-dimensional Riemannian manifold with a metric $g$. We assume that $M$ admits a $C^{\infty}$ function $f$ satisfying (5.4), namely a concircular scalar field with the characteristic function $\tau$. Let $m$ denote the number of the isolated stationary points of the gradient field $\operatorname{grad} f$. Tashiro proved the following results (cf. [24, pp. 251-257]):

(1) The stationary points are isolated and $m \leq 2$.

(2) According to $m=0,1$ and 2, $M$ is diffeomorphic to the direct product of a complete one-dimensional Riemannian manifold $Z$ and $\boldsymbol{R}$, a Euclidean space $\boldsymbol{R}^{2}$ and a twodimensional sphere $S^{2}$.

(3) The integral curves of $\operatorname{grad} f$ are geodesics. Denoting by $W$ the set of the stationary points, we can take a local coordinates $\{u, \theta\}$ on $M \backslash W$ such that $u$-curves coincide with the integral curves and $\theta$ is a local coordinate on $Z(m=0)$ or the unit circle in the tangent space at $P \in W(m=1,2)$; in other words, $\{u, \theta\}$ is the geodesic polar coordinates around $P$. Moreover, in terms of $\{u, \theta\}, f$ is represented as a function $f(u)$ of only $u$, and $g$ has the form

$$
g=d u^{2}+a(u)^{2} d \theta^{2}, \quad a(u)=c f^{\prime}(u)>0,
$$

where $c$ is some constant.

REMARK. Tashiro obtained stronger results than those stated above for $n(\geq 2)$-dimensional Riemannian manifolds.

In (3), the domain $I$ of $a$ is $(-\infty, \infty),[0, \infty)$ and $[0, L]$, respectively, if $m=0,1$ and 2, where $L=\operatorname{dist}(P, Q)$ and $W=\{P, Q\}$. Since $f^{\prime}(u) \neq 0$ on the interior of $I$, we have the inverse function $u=u(f)$ of $f$, defined on $f(I)$. The equation (5.4) implies that $\tau$ depends only on $u$. Therefore, by taking the composition of $\tau(u)$ and $u(f)$, we see that $\tau$ can be regarded as a function of $f$. Since

$$
\frac{d \tau}{d f} \nabla f=\nabla \tau=-\frac{1}{2} \nabla \triangle f=-K \nabla f,
$$

the curvature $K$ is given by

$$
K=-\frac{a^{\prime \prime}}{a}=U(f)
$$

where $U(f):=-d \tau / d f$.

We want to compute $\nabla \nabla K$ under the assumption that $d^{2} \tau / d f^{2} \neq 0$ everywhere on $f(I)$. Let ${ }^{*}$ denote differentiation with respect to $f$. Since

$$
\nabla K=\dot{U}(f) \nabla f
$$


we have, from (5.4),

$$
\begin{aligned}
\nabla \nabla K & =\ddot{U}(f) \nabla f \otimes \nabla f+\dot{U} \nabla \nabla f \\
& =\frac{\ddot{U}(f)}{\dot{U}(f)^{2}} \nabla K \otimes \nabla K+\dot{U}(f) \tau(f) g .
\end{aligned}
$$

Our assumption implies that there exists the inverse function of $U(f)$, denoted by $v$, and hence $f=v(K)$. It is easily shown that

$$
\frac{\ddot{U}(f)}{\dot{U}(f)^{2}}=-\frac{d^{2} v / d K^{2}}{d v / d K}
$$

By using $v$, we have

$$
\begin{aligned}
\dot{U}(f) \tau(f) & =-\frac{1}{d v / d K} \int U(f) d f=-\frac{1}{d v / d K} \int K \frac{d v}{d K} d K \\
& =\frac{1}{d v / d K}\left\{\int v(K) d K-K v(K)\right\} .
\end{aligned}
$$

Define $\varphi$ and $\psi$ on the range of $K$ by

$$
\varphi(K)=\frac{1}{d^{2} J / d K^{2}}\left\{J(K)-K \frac{d J}{d K}\right\}, \quad \psi(K)=-\frac{d^{3} J / d K^{3}}{d^{2} J / d K^{2}},
$$

where $J(K)=\int v(K) d K$ and the indefinite constant of the integral is chosen in such a way that $\tau(f)=\int v(K) d K-K v(K)$. Then $K$ satisfies

$$
\nabla \nabla K=\varphi(K) g+\psi(K) \nabla K \otimes \nabla K .
$$

We have shown the following.

THEOREM 5.2. If $M$ admits a concircular scalar field $f$ with the characteristic function $\tau$ and $d^{2} \tau / d f^{2} \neq 0$ anywhere on $f(I)$, then the curvature $K$ satisfies (5.8).

We finally start from the assumption that $K$ satisfies (5.8). Substituting (5.8) into the Ricci identity:

$$
\nabla_{h} \nabla_{i} \nabla_{j} K=\nabla_{i} \nabla_{h} \nabla_{j} K-K\left(\delta_{h}^{p} g_{i j}-\delta_{i}^{p} g_{h j}\right) \nabla_{p} K,
$$

we easily obtain

$$
\begin{aligned}
\dot{\varphi}(K) \nabla_{h} K g_{i j}+\dot{\psi}(K) \nabla_{h} K \nabla_{i} K \nabla_{j} K+\psi(K) \nabla_{i} K \nabla_{h} \nabla_{j} K & \\
= & \dot{\varphi}(K) \nabla_{i} K g_{h j}+\dot{\psi}(K) \nabla_{i} K \nabla_{h} K \nabla_{j} K+\psi(K) \nabla_{h} K \nabla_{i} \nabla_{j} K \\
& -K\left(\delta_{h}^{p} g_{i j}-\delta_{i}^{p} g_{h j}\right) \nabla_{p} K,
\end{aligned}
$$

where ${ }^{\cdot}$ denotes differentiation with respect to $K$. Transvecting with $g^{h j}$ and using (5.8) again, we have

$$
\left\{\dot{\varphi}(K)+K+\psi(K) \Delta K+\varphi(K) \psi(K)+\psi^{2}(K)\|\nabla K\|^{2}\right\} \nabla K=0 .
$$

Substituting

$$
\Delta K=-2 \varphi(K)-\psi(K)\|\nabla K\|^{2}
$$


into this, we obtain

$$
\dot{\varphi}(K)+K-\varphi(K) \psi(K)=0
$$

on the set of non-critical points of $K$.

Assume that $\varphi(K) \neq 0$ at an arbitrary critical point of $K$. Then the critical points are isolated and (5.9) holds on $M$. Consider a nontrivial function $v=v(K)$ satisfying

Define $J(K)$ by

$$
\frac{d^{2} v}{d K^{2}}+\psi(K) \frac{d v}{d K}=0
$$

$$
J(K)=K v(K)+\dot{v}(K) \varphi(K) .
$$

Then, in virtue of (5.9),

$$
\begin{aligned}
\dot{J}(K) & =v(K)+\{K-\varphi(K) \psi(K)+\dot{\varphi}(K)\} \dot{v}(K) \\
& =v(K) .
\end{aligned}
$$

Therefore, if we set

$$
v(K)=C \int e^{-\Psi(K)} d K, \quad \Psi(K)=\int \psi(K) d K
$$

with some non-zero constant $C$, then $J(K)$ defined by (5.10) satisfies $\dot{J}(K)=v(K)$ and hence

$$
\begin{aligned}
\nabla \nabla \dot{J}(K) & =\ddot{v}(K) \nabla K \otimes \nabla K+\dot{v}(K) \nabla \nabla K \\
& =\{J(K)-K \dot{J}(K)\} g
\end{aligned}
$$

because of (5.8) and (5.10).

THEOREM 5.3. If the Gauss curvature $K$ of a compact, orientable, two-dimensional Riemannian manifold $M$ satisfies (5.8) and $\varphi(K) \neq 0$ at any critical point of $K$, then the metric of $M$ is a critical point of the functional $\mathcal{F}_{J}$ where $\dot{J}=v$ and $v$ is defined by (5.11).

6. Surfaces admitting a concircular scalar field. All facts in this section about elliptic functions are well-known; for instance, see [1, 7, 14].

If the equation $\nabla \nabla f=\tau g$ is restricted to a geodesic, then it reduces to an ordinary differential equation $f^{\prime \prime}=\tau(f)$. When $\tau$ is a linear function of $f$, then the Riemannian manifold which admits the concircular scalar field $f$ was determined in [18, 24]. So we study the cases that $\tau$ is a polynomial of degree 2 or 3 with constant coefficients under the assumption that $M$ is a complete two-dimensional Riemannian manifold, although the results are easily generalized to the case that the dimension is not restricted.

We consider real solutions of the following differential equations with constant real coefficients:

$$
\begin{aligned}
& f^{\prime \prime}=6 f^{2}-\frac{1}{2} g_{2}, \\
& f^{\prime \prime}=\varepsilon\left(2 f^{3}+6 a_{2} f+2 a_{3}\right),
\end{aligned}
$$


where $\varepsilon= \pm 1$. Since, making use of solutions of (6.1), we can obtain those of (6.2), we first deal with (6.1). We have from (6.1)

$$
\left(f^{\prime}\right)^{2}=4 f^{3}-g_{2} f-g_{3},
$$

where $g_{3}$ is a constant real number. The roots of the polynomial

$$
p(x)=4 x^{3}-g_{2} x-g_{3}
$$

will be denoted by $e_{1}, e_{2}$ and $e_{3}$. The discriminant $D$ is given by

$$
\begin{aligned}
D & =16\left(e_{1}-e_{2}\right)^{2}\left(e_{2}-e_{3}\right)^{2}\left(e_{3}-e_{1}\right)^{2} \\
& =g_{2}{ }^{3}-27 g_{3}{ }^{2} .
\end{aligned}
$$

We have the relations:

(6.4) $e_{1}+e_{2}+e_{3}=0, \quad e_{1} e_{2}+e_{2} e_{3}+e_{3} e_{1}=-\frac{1}{4} g_{2}, \quad e_{1} e_{2} e_{3}=\frac{1}{4} g_{3}$.

Since $f$ is a function defined on $M, f$ restricted to a geodesic is defined on $\boldsymbol{R}$. So we have to exclude solutions which diverge at a finite number $t_{0} \in \boldsymbol{R}$ from nontrivial solutions of (6.1). We shall set the initial conditions for solutions in the most suitable ones in each case, so that every solution is obtained by the change of variable: $t \rightarrow t+d$.

In the case that $D>0$, the roots are real numbers. First, we assume that $D>0$ and $e_{3}<e_{2}<e_{1}$. Clearly, the real solution $f$ satifies $e_{3} \leq f \leq e_{2}$ or $e_{1} \leq f$. In the case that $e_{3} \leq f \leq e_{2}$, the solution with initial conditions $f(0)=e_{3}$ and $f^{\prime}(0)=0$ is given by

$$
f(t)=\wp\left(t+\omega^{\prime}\right),
$$

where $\wp$ is the Weierstrass elliptic function with periods $2 \omega \in \boldsymbol{R}$ and $2 \omega^{\prime} \in \sqrt{-1} \boldsymbol{R}$ (cf. [1, p. 105]):

$$
\omega=\int_{e_{1}}^{\infty} \frac{d x}{\sqrt{4 x^{3}-g_{2} x-g_{3}}}, \quad \omega^{\prime}=\sqrt{-1} \int_{-e_{3}}^{\infty} \frac{d x}{\sqrt{4 x^{3}-g_{2} x+g_{3}}} .
$$

We note that $\wp(\omega)=e_{1}, \wp\left(\omega+\omega^{\prime}\right)=e_{2}$ and $\wp\left(\omega^{\prime}\right)=e_{3}$. Using the Jacobi elliptic functions, (6.5) becomes

$$
f(t)=e_{3}+\left(e_{2}-e_{3}\right) \operatorname{sn}^{2}\left(\sqrt{e_{1}-e_{3}} t\right),
$$

where the modulus $\kappa$ is given by $\kappa^{2}=\left(e_{2}-e_{3}\right) /\left(e_{1}-e_{3}\right)$.

In the case that $e_{1} \leq f$, the solution with initial conditions $f(0)=e_{1}$ and $f^{\prime}(0)=0$ is given by

$$
f(t)=\wp(t+\omega) .
$$

In this case, we have $\lim _{t \rightarrow-\omega} f(t)=\infty$.

Second, we consider the case that $D=0$. Assume that $e_{3}<e_{2}=e_{1}$. Then the real solution $f$ satisfies $e_{3} \leq f<e_{1}$ or $f \geq e_{1}$. In the case that $e_{3} \leq f<e_{1}$, the solution with initial condition $f(0)=e_{3}$ and $f^{\prime}(0)=0$ is given by

$$
f(t)=e_{3}+\left(e_{1}-e_{3}\right) \tanh ^{2}\left(\sqrt{e_{1}-e_{3}} t\right),
$$


which is the limit solution of (6.7) as $\kappa^{2} \rightarrow 1$. We note that $\lim _{t \rightarrow \infty} f(t)=e_{1}$. If $f \geq e_{1}$, then the solution with $\lim _{t \rightarrow \infty} f(t)=e_{1}$ and $\lim _{t \rightarrow \infty} f^{\prime}(t)=0(\omega=\infty)$ is given by

$$
f(t)=e_{1}+\left(e_{1}-e_{3}\right) \frac{1}{\sinh ^{2}\left(\sqrt{e_{1}-e_{3}} t\right)},
$$

which shows that $\lim _{t \rightarrow 0} f(t)=\infty$. Next, assume that $e_{3}=e_{2}<e_{1}$. In this case, $\kappa=0$ and $\sqrt{e_{1}-e_{3}} \omega=\pi / 2$. The real solution $f$ satisfies $f \geq e_{1}$. The solution with initial condition $f(0)=e_{1}$ and $f^{\prime}(0)=0$ is given by

$$
f(t)=e_{1}+\left(e_{1}-e_{3}\right) \tan ^{2}\left(\sqrt{e_{1}-e_{3}} t\right) .
$$

Thus $\lim _{t \rightarrow t_{0}} f(t)=\infty$, where $t_{0}=\pi /\left(2 \sqrt{e_{1}-e_{3}}\right)$. Assume that $e_{1}=e_{2}=e_{3}$. From (6.4), we see that $e_{1}=e_{2}=e_{3}=0$. Therefore the solution is

$$
f(t)=\frac{1}{t^{2}},
$$

for which we have $\lim _{t \rightarrow 0} f(t)=\infty$.

Third, let us assume that $D<0$. One of the roots, say $e_{2}$, is real and the others are conjugate complex numbers. We also see that the periods $2 \omega$ and $2 \omega^{\prime}$ are conjugate complex numbers and so $\omega+\omega^{\prime}$ is real, which is given by

$$
\omega+\omega^{\prime}=-\int_{e_{2}}^{\infty} \frac{d x}{\sqrt{4 x^{3}-g_{2} x-g_{3}}}
$$

(cf. [1]). In this case, the real solution with $f(0)=e_{2}$ and $f^{\prime}(0)=0$ is given by

$$
f(t)=\wp\left(t+\omega+\omega^{\prime}\right) .
$$

Thus we have $\lim _{t \rightarrow-\left(\omega+\omega^{\prime}\right)} f(t)=\infty$. In consequence, we have

LEMMA 6.1. Among the nonconstant solutions of (6.1), the solutions which are defined on the whole line $\boldsymbol{R}$ are (6.7) in the case ( $\left.D>0, e_{3}<e_{2}<e_{1}\right)$, and (6.9) in the case ( $\left.D=0, e_{3}<e_{2}=e_{1}\right)$, up to a change of variable $: t \mapsto t+d$ and a scalar multiple of $f$.

Let us turn to the differential equation (6.2). We assume that the polynomial

$$
q(x)=x^{4}+6 a_{2} x^{2}+4 a_{3} x+a_{4}
$$

has at least a real root. We denote the minimum of the real roots by $x_{4}$. The nontrivial solutions of (6.2) satisfy

$$
\left(f^{\prime}\right)^{2}=\varepsilon q(f) .
$$

First, assume that $x_{4}$ is a simple root. Let $x_{1}, \ldots, x_{4}$ be the roots of $q(x)$. Put $f=$ $x_{4}+1 / z$. Then we have

$$
\left(z^{\prime}\right)^{2}=-\varepsilon \alpha\left(z-\alpha_{1}\right)\left(z-\alpha_{2}\right)\left(z-\alpha_{3}\right),
$$


where $\alpha=\left(x_{1}-x_{4}\right)\left(x_{2}-x_{4}\right)\left(x_{3}-x_{4}\right)>0$ and $\alpha_{i}=\left(x_{i}-x_{4}\right)^{-1}(i=1,2,3)$. Furthermore we put $z=A y+B$. Then

$$
\left(y^{\prime}\right)^{2}=-\varepsilon \alpha A\left(y+\frac{B-\alpha_{1}}{A}\right)\left(y+\frac{B-\alpha_{2}}{A}\right)\left(y+\frac{B-\alpha_{3}}{A}\right) .
$$

So if we define $A$ and $B$ by $A=-4 \varepsilon / \alpha$ and $B=\left(\alpha_{1}+\alpha_{2}+\alpha_{3}\right) / 3$, respectively, then we can rewrite (6.16) as

$$
\left(y^{\prime}\right)^{2}=4\left(y-e_{1}\right)\left(y-e_{2}\right)\left(y-e_{3}\right),
$$

where

$$
\begin{array}{lll}
e_{i}=\frac{\alpha_{i}-B}{A} & (i=1,2,3) & \text { if } \varepsilon=1, \\
e_{1}=\frac{\alpha_{3}-B}{A}, & e_{2}=\frac{\alpha_{2}-B}{A}, \quad e_{3}=\frac{\alpha_{1}-B}{A} & \text { if } \varepsilon=-1 .
\end{array}
$$

This means that a part of real solutions of (6.15) can be obtained by setting $f=x_{4}+1 /(A y+$ $B$ ) for a real solutions $y$ of (6.17). We note that $A, B \in \boldsymbol{R}$ and

$$
\begin{array}{ll}
e_{1}=\frac{1}{4}\left(2 a_{2}-x_{1} x_{4}-x_{2} x_{3}\right), \quad e_{2}=\frac{1}{4}\left(2 a_{2}-x_{2} x_{4}-x_{1} x_{3}\right), & \\
e_{3}=\frac{1}{4}\left(2 a_{2}-x_{3} x_{4}-x_{1} x_{2}\right) & \text { if } \varepsilon=1,
\end{array}
$$

$$
e_{1}=-\frac{1}{4}\left(2 a_{2}-x_{3} x_{4}-x_{1} x_{2}\right), \quad e_{2}=-\frac{1}{4}\left(2 a_{2}-x_{2} x_{4}-x_{1} x_{3}\right),
$$

$$
e_{3}=-\frac{1}{4}\left(2 a_{2}-x_{1} x_{4}-x_{2} x_{3}\right) \quad \text { if } \varepsilon=-1 .
$$

Let us consider the case that $x_{4}<x_{3}<x_{2}<x_{1}$ (real). By (6.18) we see that $e_{3}<e_{2}<$ $e_{1}$. The solutions of (6.15) corresponding to (6.7) are

$$
\begin{aligned}
& f^{+}(t)=\frac{x_{3}\left(x_{2}-x_{4}\right)-x_{4}\left(x_{2}-x_{3}\right) \operatorname{sn}^{2}\left(\sqrt{e_{1}-e_{3}} t\right)}{\left(x_{2}-x_{4}\right)-\left(x_{2}-x_{3}\right) \operatorname{sn}^{2}\left(\sqrt{e_{1}-e_{3}} t\right)}, \\
& f^{-}(t)=\frac{x_{1}\left(x_{2}-x_{4}\right)+x_{4}\left(x_{1}-x_{2}\right) \mathrm{sn}^{2}\left(\sqrt{e_{1}-e_{3}} t\right)}{\left(x_{2}-x_{4}\right)+\left(x_{1}-x_{2}\right) \operatorname{sn}^{2}\left(\sqrt{e_{1}-e_{3}} t\right)},
\end{aligned}
$$

according as $\varepsilon= \pm 1$. The solution $f^{+}$(resp. $f^{-}$) attains the minimum $x_{3}$ (resp. $x_{2}$ ) at $t=0$ (resp. $t=\omega$ ), the maximum $x_{2}$ (resp. $x_{1}$ ) at $t=\omega($ resp. $t=0$ ) and is a periodic function with period $2 \omega$. The solutions corresponding to (6.8) are

$$
\begin{aligned}
& f^{+}(t)=\frac{x_{1}\left(x_{2}-x_{4}\right)-x_{2}\left(x_{1}-x_{4}\right) \operatorname{sn}^{2}\left(\sqrt{e_{1}-e_{3}} t\right)}{\left(x_{2}-x_{4}\right)-\left(x_{1}-x_{4}\right) \operatorname{sn}^{2}\left(\sqrt{e_{1}-e_{3}} t\right)}, \\
& f^{-}(t)=\frac{x_{3}\left(x_{2}-x_{4}\right)-x_{2}\left(x_{3}-x_{4}\right) \operatorname{sn}^{2}\left(\sqrt{e_{1}-e_{3}} t\right)}{\left(x_{2}-x_{4}\right)-\left(x_{3}-x_{4}\right) \operatorname{sn}^{2}\left(\sqrt{e_{1}-e_{3}} t\right)} .
\end{aligned}
$$

The solution $\left(6.20^{+}\right)$diverges at $t=t_{0}$ such that $\operatorname{sn}^{2}\left(\sqrt{e_{1}-e_{3}} t_{0}\right)=\left(x_{2}-x_{4}\right) /\left(x_{1}-x_{4}\right)$. The solution $\left(6.20^{-}\right)$attains the maximum $x_{3}$ at $t=0$, the minimum $x_{4}$ at $t=\omega$ and is a 
peiodic function with period $2 \omega$. The function $\wp(t)$ is certainly a real solution of (6.3) which coincides with (6.8) up to a change of variable : $t \mapsto t+\omega$. The solutions corresponding to $\wp(t)$ are

$$
\begin{aligned}
& f^{+}(t)=\frac{x_{4}\left(x_{1}-x_{3}\right)-x_{3}\left(x_{1}-x_{4}\right) \operatorname{sn}^{2}\left(\sqrt{e_{1}-e_{3}} t\right)}{\left(x_{1}-x_{3}\right)-\left(x_{1}-x_{4}\right) \operatorname{sn}^{2}\left(\sqrt{e_{1}-e_{3}} t\right)}, \\
& f^{-}(t)=\frac{x_{4}\left(x_{1}-x_{3}\right)+x_{1}\left(x_{3}-x_{4}\right) \operatorname{sn}^{2}\left(\sqrt{e_{1}-e_{3}} t\right)}{\left(x_{1}-x_{3}\right)+\left(x_{3}-x_{4}\right) \operatorname{sn}^{2}\left(\sqrt{e_{1}-e_{3}} t\right)} .
\end{aligned}
$$

The solution $\left(6.21^{+}\right)$attains the maximum $x_{4}$ at $t=0$ and $\lim _{t \rightarrow t_{0}} f^{+}(t)=-\infty$, where $\operatorname{sn}^{2}\left(\sqrt{e_{1}-e_{3}} t_{0}\right)=\left(x_{1}-x_{3}\right) /\left(x_{1}-x_{4}\right)$. The solution $\left(6.21^{-}\right)$coincides with $\left(6.20^{-}\right)$up to a change of variable : $t \mapsto t+\omega$.

Next, we consider the case $x_{4}<x_{3}<x_{2}=x_{1}$. We have $e_{3}<e_{2}=e_{1}$ if $\varepsilon=1$ and $e_{3}=e_{2}<e_{1}$ if $\varepsilon=-1$. The solutions corresponding to (6.9) (i.e., the limit of $\left(6.19^{+}\right)$as $\left.\kappa^{2} \rightarrow 1\right)$ and (6.11) (the limit of $\left(6.20^{-}\right)$as $\left.\kappa \rightarrow 0\right)$ are

$$
\begin{gathered}
f^{+}(t)=\frac{x_{3}\left(x_{1}-x_{4}\right)-x_{4}\left(x_{1}-x_{3}\right) \tanh ^{2}\left(\sqrt{e_{1}-e_{3}}\right)}{\left(x_{1}-x_{4}\right)-\left(x_{1}-x_{3}\right) \tanh ^{2}\left(\sqrt{e_{1}-e_{3}}\right)}, \\
f^{-}(t)=\frac{x_{3}\left(x_{1}-x_{4}\right)-x_{1}\left(x_{3}-x_{4}\right) \sin ^{2}\left(\sqrt{e_{1}-e_{3}} t\right)}{\left(x_{1}-x_{4}\right)-\left(x_{3}-x_{4}\right) \sin ^{2}\left(\sqrt{e_{1}-e_{3}} t\right)},
\end{gathered}
$$

respectively. The function $\left(6.22^{+}\right)$satisfies $f^{+}(0)=x_{3}$, which is the minimum, and $\lim _{t \rightarrow \pm \infty} f^{+}(t)=x_{1}$. For (6.10), we can show that if $\varepsilon=1$, there exists $t_{0}$ such that $A y\left(t_{0}\right)+B=0$. Indeed, the range of the function (6.10) is $\left[e_{1}, \infty\right)$ and $-B / A=\left(a_{2}+\right.$ $\left.x_{4}{ }^{2}\right) / 2>\left(a_{2}+x_{1}{ }^{2}\right) / 2=e_{1}$ if $\varepsilon=1$. Thus we have $\lim _{t \rightarrow t_{0}}\left|f^{+}(t)\right|=\infty$ for the limit solution of $\left(6.20^{+}\right)$as $\kappa^{2} \rightarrow 1$. Since $0<\left(x_{1}-x_{3}\right) /\left(x_{1}-x_{4}\right)<1$, we also see that there exists $t_{0}$ such that $\lim _{t \rightarrow t_{0}}\left|f^{+}(t)\right|=\infty$ for the limit solution of $\left(6.21^{+}\right)$as $\kappa^{2} \rightarrow 1$. On the other hand, $\left(6.22^{-}\right)$attains the maximum $x_{3}$ at $t=0$ and the minimum $x_{4}$ at $t=\pi /\left(2 \sqrt{e_{1}-e_{3}}\right)$. The limit solution of $\left(6.21^{-}\right)$as $\kappa \rightarrow 0$ coincides with $\left(6.22^{-}\right)$up to a change of variable: $t \mapsto t+\pi /\left(2 \sqrt{e_{1}-e_{3}}\right)$.

Consider the case that $x_{4}<x_{3}=x_{2}<x_{1}$. Then we have $e_{3}=e_{2}<e_{1} \quad(\varepsilon=1)$ and $e_{3}<e_{2}=e_{1} \quad(\varepsilon=-1)$. Thus the solutions corresponding to (6.11) and (6.9) are

$$
\begin{gathered}
f^{+}(t)=\frac{x_{1}\left(x_{2}-x_{4}\right)-x_{2}\left(x_{1}-x_{4}\right) \sin ^{2}\left(\sqrt{e_{1}-e_{3}} t\right)}{\left(x_{2}-x_{4}\right)-\left(x_{1}-x_{4}\right) \sin ^{2}\left(\sqrt{e_{1}-e_{3}} t\right)}, \\
f^{-}(t)=\frac{x_{1}\left(x_{2}-x_{4}\right)+x_{4}\left(x_{1}-x_{2}\right) \tanh ^{2}\left(\sqrt{e_{1}-e_{3}}\right)}{\left(x_{2}-x_{4}\right)+\left(x_{1}-x_{2}\right) \tanh ^{2}\left(\sqrt{e_{1}-e_{3}}\right)},
\end{gathered}
$$

respectively. The solution $\left(6.23^{+}\right)$is the limit of $\left(6.20^{+}\right)$as $\kappa \rightarrow 0$. Since $0<\left(x_{2}-\right.$ $\left.x_{4}\right) /\left(x_{1}-x_{4}\right)<1$, there exists $t_{0}$ such that $\lim _{t \rightarrow t_{0}}\left|f^{+}(t)\right|=\infty$. For $\left(6.23^{+}\right)$, the function $f^{+}\left(t+\pi /\left(2 \sqrt{e_{1}-e_{3}}\right)\right)$ is the real solution which is the limit of $\left(6.21^{+}\right)$and satisfies $f^{+}\left(0+\pi /\left(2 \sqrt{e_{1}-e_{3}}\right)\right)=x_{4}$. For this solution, there exists $t_{0}$ such that $\lim _{t \rightarrow t_{0}} \mid f^{+}(t+$ 
$\left.\pi /\left(2 \sqrt{e_{1}-e_{3}}\right)\right) \mid=\infty$. The solution $\left(6.23^{-}\right)$, which is the limit of $\left(6.19^{-}\right)$as $\kappa^{2} \rightarrow 1$, attains the maximum $x_{1}$ at $t=0$ and $\lim _{t \rightarrow \pm \infty} f^{-}(t)=x_{2}$, which is the infimum. The solution $f^{-}$ which attains the minimum $x_{4}$ at $t=0$ and satisfies $\lim _{t \rightarrow \pm \infty} f^{-}(t)=x_{3}$ is given by

$$
f^{-}(t)=\frac{x_{4}\left(x_{1}-x_{3}\right)+x_{1}\left(x_{3}-x_{4}\right) \tanh ^{2}\left(\sqrt{e_{1}-e_{3}} t\right)}{\left(x_{1}-x_{3}\right)+\left(x_{3}-x_{4}\right) \tanh ^{2}\left(\sqrt{e_{1}-e_{3}}\right)},
$$

which is the limit of $\left(6.21^{-}\right)$as $\kappa^{2} \rightarrow 1$.

In the case that $x_{4}<x_{3}=x_{2}=x_{1}$, we have $e_{1}=e_{2}=e_{3}$ and hence the solutions corresponding to (6.12) are

$$
f^{+}(t)=\frac{x_{4}-4 x_{1}^{3} t^{2}}{1-4 x_{1}^{2} t^{2}}
$$

We have $\lim _{t \rightarrow \pm 1 /\left(2 x_{1}\right)}\left|f^{+}(t)\right|=\infty$ for $\left(6.25^{+}\right)$. We note that $f^{+}(t)>x_{1}$ (resp. $\left.<x_{4}\right)$ if $|t|>1 /\left(2 x_{1}\right)$ (resp. $\left.|t|<1 /\left(2 x_{1}\right)\right)$. The solution $\left(6.25^{-}\right)$attains the minimum $x_{4}$ at $t=0$ and $\lim _{t \rightarrow \pm \infty} f^{-}(t)=x_{1}$, which is the infimum.

In the case that $x_{4}<x_{2}$ and $x_{3}=\bar{x}_{1} \notin \boldsymbol{R}$, we see that $e_{2}$ is real and $e_{3}\left(=\bar{e}_{1}\right)$ is not real. The real solution with $f^{ \pm}(0)=x_{2}$ corresponds to (6.14) and that with $f^{+}(0)=x_{4}$ to $y(t)=\wp(t)$. The range of (6.14) and $\wp$ is $\left[e_{2}, \infty\right)$ and $-B / A>e_{2}$ if $\varepsilon=1$. Thus we see that $f^{+}(t)=x_{4}+1 /(A y(t)+B)$ diverges at some $t_{0}$. The solution $f^{-}$corresponding to (6.14) is given by

$$
\begin{aligned}
f^{-}(t) & =x_{4}+\frac{1}{A \wp\left(t+\omega+\omega^{\prime}\right)+B} \\
& =\frac{x_{2}\left|x_{1}-x_{4}\right|+x_{4}\left|x_{1}-x_{2}\right|+\left(x_{2}\left|x_{1}-x_{4}\right|-x_{4}\left|x_{1}-x_{2}\right|\right) \operatorname{cn}(\gamma t)}{\left|x_{1}-x_{4}\right|+\left|x_{1}-x_{2}\right|+\left(\left|x_{1}-x_{4}\right|-\left|x_{1}-x_{2}\right|\right) \operatorname{cn}(\gamma t)},
\end{aligned}
$$

(cf. [7, p. 86]), where $\gamma=\sqrt{\left|x_{1}-x_{2}\right|\left|x_{3}-x_{4}\right|}$ and the square of the modulus $\kappa$ of $\operatorname{cn}(\gamma t)$ is equal to $\left(\left|e_{1}-e_{2}\right|+\mathfrak{R}\left[e_{1}-e_{2}\right]\right) /\left(2\left|e_{1}-e_{2}\right|\right)$. The solution $f^{-}$attains the minimum $x_{4}$ at $t=\omega+\omega^{\prime}$ and the maximum $x_{2}$ at $t=0$. The period is equal to $2\left(\omega+\omega^{\prime}\right)$. We shall make use of the following equation:

$$
A=\frac{4}{\left|x_{1}-x_{4}\right|^{2}\left(x_{2}-x_{4}\right)}, \quad A e_{2}+B=\frac{1}{x_{2}-x_{4}} .
$$

Next, assume that $x_{4}$ is a real double root $\left(x_{4}=x_{3}\right)$. Consider the case that $x_{4}=x_{3}<$ $x_{2}<x_{1}$. Set $\tilde{f}(t)=-f(t)$. Then $\tilde{f}$ satisfies

$$
\left(\tilde{f}^{\prime}\right)^{2}=\varepsilon\left(\tilde{f}^{4}+6 a_{2} \tilde{f}^{2}-4 a_{3} \tilde{f}+a_{4}\right) .
$$

The roots of the polynomial $\tilde{q}(x)=x^{4}+6 a_{2} x^{2}-4 a_{3} x+a_{4}$ are $\tilde{x}_{4}=-x_{1}, \tilde{x}_{3}=-x_{2}, \tilde{x}_{2}=$ $\tilde{x}_{1}=-x_{4}$. We note that $e_{i}(i=1,2,3)$ does not change. Therefore the real solutions with 
initial condition $f^{ \pm}(0)=x_{2}$ can be obtained by making use of $\left(6.22^{+}\right)$and $\left(6.22^{-}\right)$:

$$
\begin{gathered}
f^{+}(t)=\frac{x_{2}\left(x_{1}-x_{4}\right)-x_{1}\left(x_{2}-x_{4}\right) \tanh ^{2}\left(\sqrt{e_{1}-e_{3}}\right)}{\left(x_{1}-x_{4}\right)-\left(x_{2}-x_{4}\right) \tanh ^{2}\left(\sqrt{e_{1}-e_{3}}\right)}, \\
f^{-}(t)=\frac{x_{2}\left(x_{1}-x_{4}\right)-x_{4}\left(x_{1}-x_{2}\right) \sin ^{2}\left(\sqrt{e_{1}-e_{3}}\right)}{\left(x_{1}-x_{4}\right)-\left(x_{1}-x_{2}\right) \sin ^{2}\left(\sqrt{e_{1}-e_{3}}\right)},
\end{gathered}
$$

respectively. We note that $f^{+}$attains the maximum $x_{2}$ at $t=0$ and $\lim _{t \rightarrow \pm \infty} f^{+}(t)=x_{4}$. The solution $\left(6.28^{-}\right)$attains the maximum $x_{1}$ at $t=\pi /\left(2 \sqrt{e_{1}-e_{3}}\right)$ and the minimum $x_{2}$ at $t=0$. The other real solution in this case with initial condition $f^{+}(0)=x_{1}$ (or $\left.x_{4}\right)$ satisfies $\lim _{t \rightarrow t_{0}}|f(t)|=\infty$ for some $t_{0} \in \boldsymbol{R}$.

In the case that $x_{4}=x_{3}<x_{2}=x_{1}$, we directly solve (6.15). Since $q(f) \geq 0$, there does not exist a nontrivial solution if $\varepsilon=-1$. Using the relation $x_{1}+x_{4}=0$, we have the solution with initial condition $f^{+}(0)=0$ :

$\left(6.29^{+}\right) \quad f^{+}(t)= \pm x_{1} \tanh \left(x_{1} t\right)$,

which satisfies $x_{4}<f^{+}(t)<x_{1}$. Moreover, we have the solution such that $\lim _{t \rightarrow 0}\left|f^{+}(t)\right|=$ $\infty$ :

$$
f^{+}(t)= \pm x_{1} \operatorname{coth}\left(x_{1} t\right) .
$$

We consider the case that $x_{4}=x_{3}$ and $x_{2}\left(=\bar{x}_{1}\right)$ is not real. In this case, there is not a nontrivial solution if $\varepsilon=-1$. We put $f=x_{4}+2 /\left(y+\alpha_{1}+\alpha_{2}\right)$, where $\alpha_{i}=1 /\left(x_{i}-x_{4}\right)(i=$ $1,2)$. We note that $\alpha_{2}=\bar{\alpha}_{1}$ and so $\alpha_{1}+\alpha_{2}$ is real. Then we have

$$
\left(y^{\prime}\right)^{2}=\alpha\left\{y^{2}-\left(\alpha_{2}-\alpha_{1}\right)^{2}\right\}, \quad\left(\alpha=\left(x_{1}-x_{4}\right)\left(x_{2}-x_{4}\right)>0\right) .
$$

It is easy to solve (6.31). If we put $\alpha_{2}-\alpha_{1}=\sqrt{-1} b$, then the solution is

$$
y= \pm b \sinh (\sqrt{\alpha} t) \text {. }
$$

Thus we have

$$
f^{+}(t)=x_{4}+\frac{2}{ \pm b \sinh (\sqrt{\alpha} t)+\alpha_{1}+\alpha_{2}},
$$

and hence there exists $t_{0} \in \boldsymbol{R}$ such that $f(t)$ diverges as $t \rightarrow t_{0}$.

If $x_{4}$ is a triple root, then the solutions of (6.15) are given by

$$
\begin{gathered}
f^{+}(t)=\frac{x_{1}-4 x_{4}{ }^{3} t^{2}}{1-4 x_{4}{ }^{2} t^{2}}, \\
f^{-}(t)=\frac{x_{1}+4 x_{4}{ }^{3} t^{2}}{1+4 x_{4}{ }^{2} t^{2}},
\end{gathered}
$$

We obtain these solutions by setting $\tilde{f}(t)=-f(t)$ in (6.25).

If $x_{4}$ is a real quadruple root, then

$$
f^{+}(t)=x_{4} \pm \frac{1}{t}
$$


and hence $\lim _{t \rightarrow \mp 0}\left|f^{+}(t)\right|=\infty$.

The remainding case is that the polynomial $q$ has not a real root. Since $q(f)>0,(6.15)$ has no solutions if $\varepsilon=-1$. If $x_{4}=\bar{x}_{3}=\bar{x}_{2}=x_{1}$, then $\left(f^{\prime}\right)^{2}=\left(f-x_{1}\right)^{2}\left(f-\bar{x}_{1}\right)^{2}$ and hence $f^{+}(t)=\Re x_{1} \pm\left(\Im x_{1}\right) \tan \left\{\left(\Im x_{1}\right) t+c\right\}$, which shows that there exists $t_{0}$ such that $f^{+}(t)$ diverges as $t \rightarrow t_{0}$.

Finally, we deal with the case that $x_{4}=\bar{x}_{3}, \bar{x}_{2}=x_{1}$ and $x_{1} \neq x_{4}$. We reduce

$$
\int \frac{d f}{\sqrt{\left|f-x_{4}\right|^{2}\left|f-x_{1}\right|^{2}}}= \pm t
$$

to a Jacobi normal form (cf. [1, pp. 106-109]). If $\Re x_{1}=\Re x_{4}$, then $\Re x_{1}=0$, so that (6.34) becomes

$$
\int \frac{d f}{\sqrt{\left(f^{2}+b_{1}^{2}\right)\left(f^{2}+b_{4}^{2}\right)}}= \pm t
$$

where $b_{i}=\Im x_{i}(i=1,4)$. Suppose that $\Re x_{1} \neq \Re x_{4}$. We put $c_{i}=\Re x_{i}(i=1,4)$. Let us set $f=(p y+q) /(y+1)$, where $p$ and $q(p>q)$ are roots of the equation:

$$
X^{2}+\frac{1}{2 c_{1}}\left(b_{4}^{2}-b_{1}^{2}\right) X-\frac{1}{2}\left(2 c_{1}^{2}+b_{1}^{2}+b_{4}^{2}\right)=0 .
$$

Then the integral of (6.34) becomes

$$
\begin{aligned}
& \frac{p-q}{\left|p-x_{1}\right|\left|p-x_{4}\right|} \int \frac{d y}{\sqrt{\left(y^{2}+\alpha^{2}\right)\left(y^{2}+\beta^{2}\right)}} \\
& \quad\left(\alpha=\left|\frac{q-x_{1}}{p-x_{1}}\right|, \beta=\left|\frac{q-x_{4}}{p-x_{4}}\right|\right) .
\end{aligned}
$$

Since the integrals of (6.35) and (6.36) reduce to the normal form:

$$
\int \frac{d u}{\sqrt{\left(1-u^{2}\right)\left(1-\kappa^{2} u^{2}\right)}} \quad\left(\kappa^{2}=\left(\alpha^{2}-\beta^{2}\right) / \alpha^{2}\right)
$$

by putting $y^{2}=\beta^{2} u^{2} /\left(1-u^{2}\right)$, a straightforward computation shows that

$$
f^{+}(t)=\frac{q+p \beta \operatorname{tn}(\gamma t)}{1+\beta \operatorname{tn}(\gamma t)} \quad\left(\gamma^{2}=\frac{1}{4}\left(\left|x_{4}-x_{1}\right|+\left|x_{4}-x_{2}\right|\right)^{2}\right) .
$$

In particular, there exists $t_{0}$ such that $f(t)$ diverges as $t \rightarrow t_{0}$.

Summing up, we obtain

LEMMA 6.2. Among the nonconstant solutions of (6.2), the solutions which are defined on the whole line $\boldsymbol{R}$ are, up to a change of variable $: t \mapsto t+d$ and a scalar multiple of $f,\left(6.19^{+}\right),\left(6.19^{-}\right)$and $\left(6.21^{-}\right)$in the case $\left(x_{4}<x_{3}<x_{2}<x_{1}\right),\left(6.22^{+}\right)$and $\left(6.22^{-}\right)$in the case $\left(x_{4}<x_{3}<x_{2}=x_{1}\right),\left(6.23^{-}\right)$and $\left(6.24^{-}\right)$in the case $\left(x_{4}<x_{3}=x_{2}<x_{1}\right),\left(6.25^{-}\right)$ in the case $\left(x_{4}<x_{3}=x_{2}=x_{1}\right),\left(6.26^{-}\right)$in the case $\left(x_{4}<x_{2}, x_{3}=\overline{x_{1}} \notin \boldsymbol{R}\right),\left(6.28^{+}\right)$and $\left(6.28^{-}\right)$in the case $\left(x_{4}=x_{3}<x_{2}<x_{1}\right),\left(6.29^{+}\right)$in the case $\left(x_{4}=x_{3}<x_{2}=x_{1}\right)$ and $\left(6.33^{-}\right)$in the case $\left(x_{4}=x_{3}=x_{2}<x_{1}\right)$. 
REMARK: All solutions given in Lemmas 6.1 and 6.2 are characterized by the property that their ranges coincide with open, open half or closed bounded intervals $I$ such that the infimum and supremum are real roots of the polynomial $p(x)$ or $q(x)$ and there are no real roots in the interior $I$.

Let us return to the study of the manifold $M$ admitting a concircular scalar field $f$. Tashiro [24] determined $M$ when the polynomial is of degree $\leq 1$. We consider the case that the characteristic function $\tau$ is a polynomial of $f$ whose degree is less than 3 in the following theorem.

THEOREM 6.3. Let M be a complete two-dimensional Riemannian manifold, and suppose that it admits a nontrivial concircular scalar field $f$ whose characteristic function is a polynomial of $f$. If the degree is 2 or 3 , then $M$ is homothetic to one of the following manifolds. The function $f$ given in the list coincides with the concircular scalar field $f$ up to a linear transformation: $f \mapsto \lambda f+\mu$.

[I] $(\mathrm{deg}=2) . \quad \boldsymbol{R}^{2}$ with a metric:

$$
d s^{2}=d u^{2}+\frac{1}{e_{1}-e_{3}} \tanh ^{2}\left(\sqrt{e_{1}-e_{3}} u\right) \operatorname{sech}^{4}\left(\sqrt{e_{1}-e_{3}} u\right) d \theta^{2}
$$

in terms of the geodesic polar coordinates $\{u, \theta\}$ in $\boldsymbol{R}^{2}$, where $e_{1}>e_{3}$ and $2 e_{1}+e_{3}=0$. It is isometric to the surface of revolution which is obtained by rotating the unit speed curve:

$$
\begin{aligned}
& x(u)=\frac{1}{\sqrt{e_{1}-e_{3}}} \tanh \left(\sqrt{e_{1}-e_{3}} u\right) \operatorname{sech}^{2}\left(\sqrt{e_{1}-e_{3}} u\right), \\
& z(u)=\frac{1}{6 e_{1}^{2}} \int_{e_{3}}^{f(u)} \frac{1}{e_{1}-\xi} \sqrt{\left(2 e_{1}-\xi\right)\left(2 e_{1}^{2}+\xi^{2}\right)} d \xi
\end{aligned}
$$

in the $x$-z plane around the $z$-axis in $\boldsymbol{R}^{3}$, where $f(u)=e_{3}+\left(e_{1}-e_{3}\right) \tanh ^{2}\left(\sqrt{e_{1}-e_{3}} u\right)$.

[II] (deg = 3). (1) $\boldsymbol{R} \times Z$ with a warped product metric:

$$
d s^{2}=d u^{2}+x_{1}^{4} \operatorname{sech}^{4}\left(x_{1} u\right) d \theta^{2},
$$

where $\theta$ is a local coordinate in a complete one-dimensional manifold $Z, x_{1}$ is a positive constant and $f^{+}(u)=x_{1} \tanh \left(x_{1} u\right)$.

(2) $\boldsymbol{R}^{2}$ with a metric:

$$
d s^{2}=d u^{2}+a(u)^{2} d \theta^{2},
$$

where $\{u, \theta\}$ is the geodesic polar coordinates in $\boldsymbol{R}^{2}$. The function a is one of the following:

(i)

$$
\begin{aligned}
a(u) & =\frac{2\left(f^{+}\right)^{\prime}(u)}{\left(x_{3}-x_{1}\right)^{2}\left(x_{3}-x_{4}\right)}, \\
f^{+}(u) & =\frac{x_{3}\left(x_{1}-x_{4}\right)-x_{4}\left(x_{1}-x_{3}\right) \tanh ^{2}(\gamma u)}{\left(x_{1}-x_{4}\right)-\left(x_{1}-x_{3}\right) \tanh ^{2}(\gamma u)}, \\
\gamma & =\sqrt{e_{1}-e_{3}}=\frac{1}{2} \sqrt{\left(x_{1}-x_{3}\right)\left(x_{1}-x_{4}\right)},
\end{aligned}
$$


where $x_{1}, x_{3}, x_{4}$ are constants satisfying $x_{4}<x_{3}<x_{1}$ and $2 x_{1}+x_{3}+x_{4}=0$,

(ii)

$$
\begin{aligned}
a(u) & =\frac{-2\left(f^{-}\right)^{\prime}(u)}{\left(x_{3}-x_{1}\right)^{2}\left(x_{1}-x_{4}\right)}, \\
f^{-}(u) & =\frac{x_{1}\left(x_{3}-x_{4}\right)+x_{4}\left(x_{1}-x_{3}\right) \tanh ^{2}(\gamma u)}{\left(x_{3}-x_{4}\right)+\left(x_{1}-x_{3}\right) \tanh ^{2}(\gamma u)}, \\
\gamma & =\sqrt{e_{1}-e_{3}}=\frac{1}{2} \sqrt{\left(x_{1}-x_{3}\right)\left(x_{3}-x_{4}\right)},
\end{aligned}
$$

where $x_{1}, x_{3}, x_{4}$ are constants satisfying $x_{4}<x_{3}<x_{1}$ and $x_{1}+2 x_{3}+x_{4}=0$,

(iii)

$$
\begin{aligned}
a(u) & =\frac{2\left(f^{-}\right)^{\prime}(u)}{\left(x_{1}-x_{4}\right)^{3}}, \\
f^{-}(u) & =\frac{x_{4}+4 x_{1}^{3} u^{2}}{1+4 x_{1}^{2} u^{2}},
\end{aligned}
$$

where $x_{1}, x_{4}$ are constants satisfying $x_{4}<x_{1}$ and $3 x_{1}+x_{4}=0$.

(3) $S^{2}$ with a metric:

$$
d s^{2}=d u^{2}+a(u)^{2} d \theta^{2}
$$

in terms of the geodesic polar coordinates $\{u, \theta\}$ whose center is a critical point of $f$. The function a is one of the following:

(i)

$$
\begin{aligned}
a(u) & =\frac{\left(f^{+}\right)^{\prime}(u)}{x_{2}\left(x_{1}^{2}-x_{2}^{2}\right)}, \\
f^{+}(u) & =x_{4}+\frac{1}{A \wp\left(u+\omega^{\prime}\right)+B}=\frac{-x_{2}\left\{x_{1}+x_{2}-2 x_{1} \operatorname{sn}^{2}(\gamma u)\right\}}{\left(x_{1}+x_{2}\right)-2 x_{2} \operatorname{sn}^{2}(\gamma u)}, \\
\gamma & =\frac{1}{2}\left(x_{1}+x_{2}\right),
\end{aligned}
$$

where $0<x_{2}<x_{1}, A=-2 /\left\{x_{1}\left(x_{1}^{2}-x_{2}^{2}\right)\right\}, B=\left(5 x_{1}^{2}-x_{2}^{2}\right) /\left\{6 x_{1}\left(x_{1}^{2}-x_{2}^{2}\right)\right\}$ and the modulus of the Jacobi elliptic function sn is equal to $2 x_{1} x_{2} /\left(x_{1}+x_{2}\right)$.

(ii)

$$
\begin{aligned}
a(u) & =\frac{-\left(f^{-}\right)^{\prime}(u)}{x_{2}\left|x_{1}-x_{2}\right|^{2}}, \\
f^{-}(u) & =x_{4}+\frac{1}{A \wp\left(u+\omega+\omega^{\prime}\right)+B}=x_{2} \operatorname{cn}(\gamma u), \\
\gamma & =\left|x_{1}-x_{2}\right|,
\end{aligned}
$$

where $x_{2}>0, x_{1}(\neq 0)$ is a pure imaginary, $A=2 /\left(x_{2}\left|x_{1}+x_{2}\right|^{2}\right), B=\left(5 x_{2}^{2}-x_{1}^{2}\right) /\left(6 x_{2} \mid x_{1}+\right.$ $\left.\left.x_{2}\right|^{2}\right)$ and the modulus of the Jacobi elliptic function $\mathrm{cn}$ is equal to $x_{2} /\left|x_{1}+x_{2}\right|$. 
PROOF. Recall the results proved by Tashiro ((1) through (3) in Section 5). The integral curves of grad $f$ are geodesics. When the concircular scalar field $f$ is restricted to a geodesic, it satisfies (6.1) (resp. (6.2)), after a suitable linear transformation $f \mapsto \lambda f+\mu$, if the degree of the polynomial $\tau(f)$ is 2 (resp. 3). Thus $f$ restricted to a geodesic is one of the solutions given in Lemmas 6.1 and 6.2.

Suppose that the degree of $\tau(f)$ is equal to 2. Then we see from Lemma 6.1 that the number $m$ of the critical points of the concircular scalar field $f$ is 1 or 2 . If $m=1$, then $f$ restricted to the geodesic $\gamma$ which coincides with the integral curve of $\operatorname{grad} f$ is given by (6.9). If $m=2$, then $\left.f\right|_{\gamma}$ is given by (6.7). Since $d s^{2}=d u^{2}+a(u)^{2} d \theta^{2}$ (see (3) in Section 5) and the metric $d s^{2}(=g)$ is smooth at the critical point $P \in W$, we require the function $a$ to satisfy

$$
a(0)=0, \quad a^{\prime}(0)=1, \quad a^{(2 k)}(0)=0 \quad(k=1,2, \ldots),
$$

(see [2, p. 96] and [16, Proposition 2.7]). If $\left.f\right|_{\gamma}$ is given by (6.9), then $a=c f^{\prime}$ is an odd function and satisfies the condition $a^{\prime}(0)=1$ by setting $c=1 /\left\{2\left(e_{1}-e_{3}\right)^{2}\right\}$. Let $\left.f\right|_{\gamma}$ be given by (6.7). Since we have from (6.4)

$$
\begin{aligned}
a^{\prime}(0) & =c \wp^{\prime \prime}\left(\omega^{\prime}\right)=c\left(6 \wp\left(\omega^{\prime}\right)^{2}-\frac{1}{2} g_{2}\right) \\
& =2 c\left(e_{1}-e_{3}\right)\left(e_{2}-e_{3}\right),
\end{aligned}
$$

we have to put $c=1 /\left\{2\left(e_{1}-e_{3}\right)\left(e_{2}-e_{3}\right)\right\}$. Furthermore, since $d s^{2}$ gives the smooth metric $g$ at another critical point $Q \in W$, we also require $a$ to satisfy

$$
a(\omega)=0, \quad a^{\prime}(\omega)=-1, \quad a^{(2 k)}(\omega)=0 \quad(k=1,2, \ldots) .
$$

However we have

$$
\wp^{\prime \prime}\left(\omega+\omega^{\prime}\right)=2\left(e_{2}-e_{3}\right)\left(e_{2}-e_{1}\right)
$$

and hence

$$
\begin{aligned}
a^{\prime}(\omega) & =c f^{\prime \prime}(\omega) \\
& =-\frac{e_{1}-e_{2}}{e_{1}-e_{3}}=\kappa^{2}-1>-1 .
\end{aligned}
$$

We conclude that the case $m=2$ does not occur if the degree is equal to 2 . It is easy to see that $\boldsymbol{R}^{2}$ with metric given in [I] is isometric to a surface of revolution.

Suppose that the degree of $\tau(f)$ is equal to 3. If $m=0$, then $\left.f\right|_{\gamma}$ is the function given in $\left(6.29^{+}\right)$and $M$ is isometric to $\boldsymbol{R} \times Z$ with warped product metric given in [II](1)(i).

Assume that $m=1$. Then $\left.f\right|_{\gamma}$ is one of the functions given by $\left(6.22^{+}\right),\left(6.23^{-}\right)$, $\left(6.24^{-}\right),\left(6.25^{-}\right),\left(6.28^{+}\right)$and $\left(6.33^{-}\right)$. By replacing $f$ with $-f$, we see that the solutions 
$\left(6.28^{+}\right),\left(6.24^{-}\right)$and $\left(6.33^{-}\right)$are essentially the same as $\left(6.22^{+}\right),\left(6.23^{-}\right),\left(6.25^{-}\right)$, respectively. Therefore, if we set

$$
c= \begin{cases}2 /\left\{\left(x_{3}-x_{1}\right)^{2}\left(x_{3}-x_{4}\right)\right\} & \text { for }\left(6.22^{+}\right), \\ -2 /\left\{\left(x_{1}-x_{3}\right)^{2}\left(x_{1}-x_{4}\right)\right\} & \text { for }\left(6.23^{-}\right), \\ -2 /\left(x_{4}-x_{1}\right)^{3} & \text { for }\left(6.25^{-}\right),\end{cases}
$$

so that $a$ satisfies (6.38), we obtain the cases [II](2)(i), (ii) and (iii).

Next, assume that $m=2$. The function $\left.f\right|_{\gamma}$ is one of the functions given by $\left(6.19^{+}\right)$, $\left(6.19^{-}\right),\left(6.21^{-}\right),\left(6.22^{-}\right),\left(6.26^{-}\right)$and $\left(6.28^{-}\right)$. By replacing $f$ with $-f$, we see that the solutions $\left(6.21^{-}\right)$and $\left(6.28^{-}\right)$are essentially the same as $\left(6.19^{-}\right)$and $\left(6.22^{-}\right)$, respectively. In order that $a$ satisfies (6.38), it is necessary that the constant $c$ satisfies

$$
c= \begin{cases}2 /\left\{\left(x_{1}-x_{3}\right)\left(x_{2}-x_{3}\right)\left(x_{3}-x_{4}\right)\right\} & \text { for }\left(6.19^{+}\right), \\ -2 /\left\{\left(x_{1}-x_{2}\right)\left(x_{1}-x_{3}\right)\left(x_{1}-x_{4}\right)\right\} & \text { for }\left(6.19^{-}\right), \\ -2 /\left\{\left(x_{1}-x_{3}\right)^{2}\left(x_{3}-x_{4}\right)\right\} & \text { for }\left(6.22^{-}\right), \\ -2 /\left\{\left(x_{2}-x_{4}\right)\left|x_{1}-x_{2}\right|^{2}\right\} & \text { for }\left(6.26^{-}\right) .\end{cases}
$$

We denote by $2 L$ the period of the even functions $\left(6.19^{+}\right),\left(6.19^{-}\right),\left(6.22^{-}\right)$and $\left(6.26^{-}\right)$. At another critical point where $u=L$, we require

$$
a(L)=0, \quad a^{\prime}(L)=-1, \quad a^{(2 k)}(L)=0 \quad(k=1,2, \ldots),
$$

(see [2, p. 96]). We can easily show that the above four solutions satisfy (6.39) except for $a^{\prime}(L)=-1$. We first consider $\left(6.19^{+}\right)$. Noting that $L=\omega, x_{1}+x_{2}+x_{3}+x_{4}=0$ and

$$
a^{\prime}(\omega)=c f^{\prime \prime}(\omega)=-\frac{\left(x_{2}-x_{1}\right)\left(x_{2}-x_{4}\right)}{\left(x_{3}-x_{1}\right)\left(x_{3}-x_{4}\right)},
$$

we see that $a^{\prime}(L)=-1$ if and only if $x_{3}=-x_{2}$. Thus $M$ is diffeomorphic to $S^{2}$ and

$$
\begin{aligned}
a(u) & =\frac{\left(f^{+}\right)^{\prime}(u)}{x_{2}\left(x_{1}^{2}-x_{2}^{2}\right)}, \\
f^{+}(u) & =\frac{-x_{2}\left\{x_{1}+x_{2}-2 x_{1} \operatorname{sn}^{2}\left(\left(x_{1}+x_{2}\right) u / 2\right)\right\}}{\left(x_{1}+x_{2}\right)-2 x_{2} \operatorname{sn}^{2}\left(\left(x_{1}+x_{2}\right) u / 2\right)},
\end{aligned}
$$

where we note that $\sqrt{e_{1}-e_{3}}=\left(x_{1}+x_{2}\right) / 2$. We have obtained the case [II](3)(i). We then consider $\left(6.19^{-}\right)$. Noting that $L=\omega$ and

$$
a^{\prime}(\omega)=c f^{\prime \prime}(\omega)=-\frac{\left(x_{2}-x_{3}\right)\left(x_{2}-x_{4}\right)}{\left(x_{1}-x_{3}\right)\left(x_{1}-x_{4}\right)},
$$

we have $x_{2}=-x_{1}$ and $x_{4}=-x_{3}$ from the equation $a^{\prime}(L)=-1$. However, since $x_{4}<x_{3}<$ $x_{2}<x_{1}$, this case does not occur. We now consider $\left(6.22^{-}\right)$. Noting that $L=\pi /\left(2 \sqrt{e_{1}-e_{3}}\right)$ and

$$
a^{\prime}(L)=c f^{\prime \prime}(L)=-\frac{\left(x_{1}-x_{4}\right)^{2}}{\left(x_{1}-x_{3}\right)^{2}},
$$


we see that $x_{4}+x_{3}=2 x_{1}$ if and only if $a^{\prime}(L)=-1$. Since $2 x_{1}+x_{3}+x_{4}=0$ and $x_{4}<x_{3}<x_{2}=x_{1}$, this case deos not occur. Finally, we consider $\left(6.26^{-}\right)$. Noting that $L=\omega+\omega^{\prime}$ and using (6.27), we have

$$
a^{\prime}(L)=c f^{\prime \prime}(L)=-\frac{\left|x_{1}-x_{4}\right|^{2}}{\left|x_{1}-x_{2}\right|^{2}} .
$$

Thus $a^{\prime}(L)=-1$ if and only if $x_{4}=-x_{2}(\neq 0)$. Since $x_{3}=\bar{x}_{1}$ and $x_{1}+x_{2}+x_{3}+x_{4}=0$, we see that $x_{1}\left(=-x_{3}\right)$ is a pure imaginary. Thus $M$ is diffeomorphic to $S^{2}$ and $\left(6.26^{-}\right)$becomes

$$
f^{-}(u)=x_{4}+\frac{1}{A \wp\left(u+\omega+\omega^{\prime}\right)+B}=x_{2} \operatorname{cn}(\gamma u) .
$$

This case is [II](3)(ii).

We have completed to examine all cases.

COROLlary. Let $M$ be a complete two-dimensional Riemannian manifold. If the Gauss curvature $K$ satisfies $\nabla \nabla K=\tau g$, then $K$ is constant or $M$ is isometric to $\boldsymbol{R}^{2}$ with the metric whose curvature is given by $K=-x^{\prime \prime} / x, x$ being the function given in [I] of Theorem 6.3 .

Proof. Since $\nabla \nabla K=\tau g$, we have $-\Delta K=2 \tau$ and $\nabla \Delta K=2 K \nabla K=\nabla K^{2}$. It follows that $-2 \tau=K^{2}-\lambda$, where $\lambda$ is a constant. Thus we have

$$
\nabla \nabla K=-\frac{1}{2}\left(K^{2}-\lambda\right) g .
$$

We put $f=-K / 12$ and $g_{2}=\lambda / 12$. Then (6.40) becomes

$$
\nabla \nabla f=\left(6 f^{2}-\frac{1}{2} g_{2}\right) g \text {. }
$$

The characteristic function $\tau(f)$ is a polynomial of $f$ of degree 2. Thus the assertion is obtained from Theorem 6.3.

Now we consider the functional on $\mathcal{M}_{1}$ :

$$
\mathcal{F}_{2}[g]=c \int_{M}(\delta-K)^{2} d v_{g} \quad(c \neq 0),
$$

$\delta$ being a constant. Suppose that $g$ is a critical point of $\mathcal{F}_{2}$. Then $f=\dot{J}(K)=2 c(K-\delta)$ is a concircular scalar field if $K$ is not constant. Thus, from the above Corollary, we conclude the following:

THEOREM 6.4. Let $M$ be a compact orientable two-dimensional Riemannian manifold. If the metric $g$ of $M$ is critical with respect to $\mathcal{F}_{2}$, then the Gauss curvature is constant.

Next for a given real number $\delta$, we consider the functional on $\mathcal{M}_{K, \delta}^{\varepsilon}=\{g \in \mathcal{M} \mid 0 \leq$ $\delta-\varepsilon K$ on $M\}$ :

$$
\mathcal{F}_{3 / 2}^{\varepsilon}[g]=c \int_{M}(\delta-\varepsilon K)^{3 / 2} d v_{g} \quad(c \neq 0)
$$


If the metric $g$ is critical for the functional $\mathcal{F}_{3 / 2}^{\varepsilon}$, then $f=\dot{J}(K)$ satisfies (5.3) and hence

$$
\nabla \nabla f=\varepsilon\left(\frac{4}{27 c^{2}} f^{3}-\delta f\right) g .
$$

Thus it is convenient to put $c=\sqrt{2} /(3 \sqrt{3})$. If the metric $g$ is critical for the functional $\mathcal{F}_{3 / 2}^{\varepsilon} \mid \mathcal{M}_{1}$, then

$$
\nabla \nabla f=\varepsilon\left(2 f^{3}-\delta f+2 a_{3}\right) g,
$$

where the constant $a_{3}$ is chosen in such a way that $\int_{M}\left(2 f^{3}-\delta f+2 a_{3}\right) d v_{g}=0$.

THEOREM 6.5. Let M be a manifold diffeomorphic to $S^{2}$. Let $\delta$ be a real number such that $\delta \geq 4 \pi$ if $\varepsilon=1$ and $|\delta|<4 \pi$ if $\varepsilon=-1$. If a metric $g$ on $M$ is critical with respect to $\mathcal{F}_{3 / 2}^{\varepsilon} \mid \mathcal{M}_{1}$, then the Gauss curvature of $g$ is equal to $4 \pi$ (constant), $g$ is the metric given in [II](3)(i) of Theorem 6.3, where $x_{1}=\sqrt{\delta+4 \pi} / \sqrt{2}$ and $x_{2}=\sqrt{\delta-4 \pi} / 2$, or the metric given in [II](3)(ii), where $x_{1}=\sqrt{-1} \sqrt{4 \pi-\delta} / \sqrt{2}$ and $x_{2}=\sqrt{4 \pi+\delta} / \sqrt{2}$. They are on the boundary of $\mathcal{M}_{K, \delta}^{\varepsilon} \cap \mathcal{M}_{1}$.

Proof. Since $M$ is diffeomorphic to $S^{2}$, it suffies for the proof to consider the solutions, $f^{+}$in [II](3)(i) and $f^{-}$in [II](3)(ii), of the equation (6.45). In both cases, we have $a_{3}=0, \delta=x_{1}{ }^{2}+x_{2}{ }^{2}$. Using $K=f^{\prime \prime \prime} / f^{\prime}$, we also have $6 f^{2}=\delta-\varepsilon K$. Since the metric is normalized as $\operatorname{Vol}(M)=1$, we have, by the equation $a=c f^{\prime}$,

$$
\begin{aligned}
1 & =\operatorname{Vol}(M)=\int_{0}^{2 \pi} \int_{0}^{L} a(u) d u d \theta \\
& = \begin{cases}4 \pi /\left(x_{1}{ }^{2}-x_{2}{ }^{2}\right) & (\varepsilon=1), \\
4 \pi /\left(\left|x_{1}-x_{2}\right|^{2}\right) & (\varepsilon=-1) .\end{cases}
\end{aligned}
$$

It follows that $x_{1}$ and $x_{2}$ are equal to the values in the assertion. Since the maximum of $K$ is equal to $\delta$ in the case [II](3)(i) and the minimum of $K$ is equal to $-\delta$ in the case [II](3)(ii), the metrics are on the boundary of $\mathcal{M}_{K, \delta}^{\varepsilon} \cap \mathcal{M}_{1}$.

Since $J(K)=c(\delta-\varepsilon K)^{3 / 2}, K$ satisfies

$$
\nabla \nabla K=\frac{2}{3}(\delta-\varepsilon K)(2 \delta+\varepsilon K) g-\frac{\varepsilon}{2(\delta-\varepsilon K)} \nabla K \otimes \nabla K,
$$

(cf. (5.8)). However, the coefficient of $\nabla K \otimes \nabla K$ has a singular point where $K=\varepsilon \delta$ and $K$ is critical. The coefficient $(=\varphi(K)$ in (5.8)) of $g$ vanishes at the critical point. It seems to be interesting that we determine complete two-dimensional Riemannian manifolds whose Gauss curvature $K$ satisfies (6.46), or, more generally, complete Riemannian manifolds which admit a function satisfying the equation of the type (5.8), (where we should assume that $\psi(f)$ possesses a singular point). 


\section{REFERENCES}

[ 1 ] N. I. AkHIEZeR, Elements of the Theory of Elliptic Functions, Transl. Math. Monogr. 79, Amer. Math. Soc., Providence, RI, 1990.

[ 2 ] A. L. BESSE, Manifolds all of whose Geodesics are Closed, Springer-Verlag, Berlin, Heidelberg, New York, 1978.

[ 3 ] A. L. Besse, Einstein Manifolds, Springer-Verlag, Berlin, Heidelberg, New York, London, Paris, Tokyo, 1981.

[ 4 ] H. W. BRInKmAnN, Einstein spaces which are mapped conformally on each other, Math. Ann. 94 (1925), $119-145$.

[ 5 ] R. BRYANT, A duality for Willmore surfaces, J. Differential Geom. 20 (1984), $23-53$.

[ 6 ] R. BRyant, Minimal surfaces of constant curvature in $S^{n}$, Trans. Amer. Math. Soc. 290 (1985), 259-271.

[ 7 ] P. F. ByRd AND M. D. Friedman, Handbook of Elliptic Integrals for Engineers and Scientist, Second edition, Die Grundlehren der mathematischen Wissenschaften 67, Springer-Verlag, Berlin, Heidelberg, New York, 1971.

[ 8 ] E. CALABI, Minimal immersions of surfaces in Euclidean spheres, J. Differential Geom. 1 (1967), 111-125.

[9] B. Y. CHEN, Geometry of Submanifolds, Marcel Dekker, New York, 1973.

[10] N. EJIRI, A counter example for Weiner's open question, Indiana Univ. Math. J. 31 (1982), 209-211.

[11] N. EJIRI, Willmore surfaces with a duality in $S^{n}(1)$, Proc. London Math. Soc. 57 (1988), 383-416.

[12] I. V. Guadalupe AND L. RodrigueZ, Normal curvature of surfaces in space forms, Pacific J. Math. 106 (1983), 95-102.

[13] F. HÉLEIN, Willmore immersions and loop groups, J. Differential Geom. 50 (1998), 331-385.

[14] A. Hurwitz AND R. Courant, Vorlesungen über allgemeinen Funktionentheorie und elliptische Funktionen, Springer-Verlag, Berlin, Göttingen, Heidelberg, New York, 3 edition, 1929.

[15] S. ISHIHARA AND Y. TASHIRO, On Riemannian manifolds admitting a concircular transformation, Math. J. Okayama Univ. 9 (1959), 19-47.

[16] J. L. KAZDAN AND F. W. WARnER, Curvature functions for open 2-manifolds, Ann. of Math. (2) 99 (1974), 203-219.

[17] K. Kenmotsu, On minimal immersions of $R^{2}$ into $S^{n}$, J. Math. Soc. Japan 28 (1976), 182-191.

[18] M. ОватA, Certain conditions for a Riemannian manifold to be isometric with a sphere, J. Math. Soc. Japan 14 (1962), 333-340.

[19] T. Petrie and J. Randall, Connection, Definite Forms, and Four-manifolds, Oxford Sci. Publ., 1990.

[20] U. PINKALL, Inequalities of Willmore type for submanifolds, Math. Z. 193 (1986), 241-246.

[21] M. RIgoli, The conformal Gauss map of submanifolds of the Möbius space, Ann. Global Anal. Geom. 5 (1987), 97-116.

[22] M. Rigoli AND I. M. SalavesSA, Willmore submanifolds of the Möbius space and a Bernstein-type theorem, Manuscripta Math. 81 (1993), 203-222.

[23] K. S Акамото, Constant isotropic surfaces in 5-dimensional space forms, Geom. Dedicata 29 (1989), 293306.

[24] Y. TAShiro, Complete Riemannian manifolds and some vector fields, Trans. Amer. Math. Soc. 117 (1965), 251-275.

[25] J. L. WeINER, On a problem of Chen, Willmore, et al., Indiana Univ. Math. J. 27 (1978), 19-35.

[26] T. J. Willmore, Riemaniann Geometry, Oxford Sci. Publ., The Clarendon Press, Oxford University Press, New York, 1993.

[27] S. T. YAU, Submanifolds with constant mean curvature I, Amer. J. Math. 96 (1974), 346-366. 
DEPARTMENT OF MATHEMATICS

FACULTY OF SCIENCE

SAITAMA UNIVERSITY

SHIMO-OKUBO, SAITAMA

$338-8570$

JAPAN

E-mail address: ksakamot@rimath.saitama-u.ac.jp 